\title{
Relative Perturbation Theory: (I) Eigenvalue and Singular Value Variations *
}

\author{
Ren-Cang Li \\ Mathematical Science Section \\ Oak Ridge National Laboratory \\ P.O. Box 2008, Bldg 6012 \\ Oak Ridge, TN 37831-6367 \\ (li@msr.epm.ornl.gov) \\ LAPACK working notes \# 84 (first published July, 1994, revised January, 1996)
}

\begin{abstract}
The classical perturbation theory for matrix eigenvalue and singular value problems provides bounds on the absolute differences between approximate eigenvalues (singular values) and the true eigenvalues (singular values) of a matrix. These bounds may be bad news for small eigenvalues (singular values), which thereby suffer worse relative uncertainty than large ones. However, there are situations where even small eigenvalues are determined to high relative accuracy by the data, much more accurately than the classical perturbation theory would indicate. In this paper, we study how eigenvalues of a matrix $A$ change when it is perturbed to $\widetilde{A}=D_{1}^{*} A D_{2}$ and how singular values of a (nonsquare) matrix $B$ change when it is perturbed to $\widetilde{B}=D_{1}^{*} B D_{2}$, where $D_{1}$ and $D_{2}$ are assumed to be close to unitary matrices of suitable dimensions. It is proved that under these kinds of perturbations, small eigenvalues (singular values) suffer relative changes no worse than large eigenvalues (singular values). We have been able to extend many well-known perturbation theorems, including Hoffman-Wielandt theorem and Weyl-Lidskii theorem. As applications, we obtained bounds for perturbations of graded matrices in both singular value problems and nonnegative definite Hermitian eigenvalue problems.
\end{abstract}

\footnotetext{
*This material is based in part upon work supported, during January, 1992-August, 1995, by Argonne National Laboratory under grant No. 20552402 and by the University of Tennessee through the Advanced Research Projects Agency under contract No. DAAL03-91-C-0047, by the National Science Foundation under grant No. ASC-9005933, and by the National Science Infrastructure grants No. CDA-8722788 and CDA-9401156, and supported, since August, 1995, by a Householder Fellowship in Scientific Computing at Oak Ridge National Laboratory, supported by the Applied Mathematical Sciences Research Program, Office of Energy Research, United States Department of Energy contract DE-AC05-96OR22464 with Lockheed Martin Energy Research Corp. Part of this work was done during summer of 1994 while the author was at Department of Mathematics, University of California at Berkeley.
} 


\section{Introduction}

The classical perturbation theory for matrix eigenvalue problems provides bounds on the absolute differences $|\lambda-\tilde{\lambda}|$ between approximate eigenvalues $\tilde{\lambda}$ and the true eigenvalues $\lambda$ of a symmetric matrix $A$. When $\tilde{\lambda}$ is computed using standard numerical software, the bounds on $|\lambda-\tilde{\lambda}|$ are typically only moderately bigger than $\epsilon\|A\|[14,33,42]$, where $\epsilon$ is the rounding error threshold characteristics of the computer's arithmetic. These bounds are bad news for small eigenvalues, which thereby suffer worse relative uncertainty than large ones.

Generally, the classical error bounds are best possible if perturbations are arbitrary. However, there are situations where perturbations have special structures and under these special perturbations even small eigenvalues (singular values) are determined to high relative accuracy by the data, much more accurately than the classical perturbation theory would indicate. The relative perturbation theory is then called for to exploit the situations to provide bounds on the relative differences between $\tilde{\lambda}$ and $\lambda$.

The development of such a theory went back to as early as Kahan $[18,1966]$ and is becoming a very active research area in the last six years or so and ever since $[1,6,7,8$, $9,10,11,13,15,21,29,34]$. In this paper, we develop a theory by a unifying treatment that sharpens existing bounds and covers almost all previously studied cases.

\subsection{What to be Covered?}

This paper deals with perturbations of the following kinds:

\section{- Eigenvalue problems:}

1. $A$ and $\widetilde{A}=D^{*} A D$ for Hermitian case, where $D$ is nonsingular and close to the identity matrix or more generally to a unitary matrix;

2. $A$ and $\tilde{A}=D_{1}^{*} A D_{2}$ for general diagonalizable case, where $D_{1}$ and $D_{2}$ are nonsingular and close to the identity matrix or more generally to some unitary matrix;

3. $A=S^{*} H S$ and $\tilde{A}=S^{*} \tilde{H} S$ for the graded nonnegative Hermitian case, where it is assumed that $H$ and $\widetilde{H}$ are nonsingular and often that $S$ is a highly graded diagonal matrix (this assumption is not necessary to our theorems).

\section{- Singular value problems:}

1. $B$ and $\widetilde{B}=D_{1}^{*} B D_{2}$, where $D_{1}$ and $D_{2}$ are nonsingular and close to identity matrices or more generally to unitary matrices;

2. $B=G S$ and $\widetilde{B}=\widetilde{G} S$ for the graded case, where it is assumed that $G$ and $\widetilde{G}$ are nonsingular and often that $S$ is a highly graded diagonal matrix (this assumption is not necessary to our theorems).

These perturbations cover component-wise relative perturbations to entries of symmetric tridiagonal matrices with zero diagonal $[8,18]$, entries of bidiagonal and biacyclic matrices 
$[1,7,8]$, and perturbations in graded nonnegative Hermitian matrices [9, 29], in graded matrices of singular value problems $[9,29]$ and more [10]. What distinguishes these perturbations from the most general additive perturbations studied by the classical perturbation theory is their multiplicative structures. For this reason, we call such perturbations multiplicative perturbations. (The above perturbations for graded matrices can be transformed to take forms of multiplicative perturbations as will be seen from proofs of this paper.)

Additive perturbations are the most general in the sense that if $A$ is perturbed to $\tilde{A}$, only possible known information is on some norm of $\Delta A \stackrel{\text { def }}{=} \tilde{A}-A$. Such perturbations, no matter how small, can not guarantee relative accuracy in eigenvalues (singular values) of the matrix under considerations. For example, $A$ is singular, then $\widetilde{A}$ can be made nonsingular no matter how small a norm of $\Delta A$ is; thus some zero eigenvalues are perturbed to nonzero ones and therefore lose their relative accuracy completely. (Retaining any relative accuracy of zero at all ends up not changing it.)

\subsection{Notation}

We will adopt this convention: capital letters denote unperturbed matrices and capital letters with tilde denote their perturbed ones. For example, $X$ is perturbed to $\tilde{X}$.

Throughout the paper, capital letters are for matrices, lower case Latin letters for column vectors or scalars, and lower case Greek letters for scalars. The following is a detailed list of our special notation. Others will be introduced when it appears for the first time.

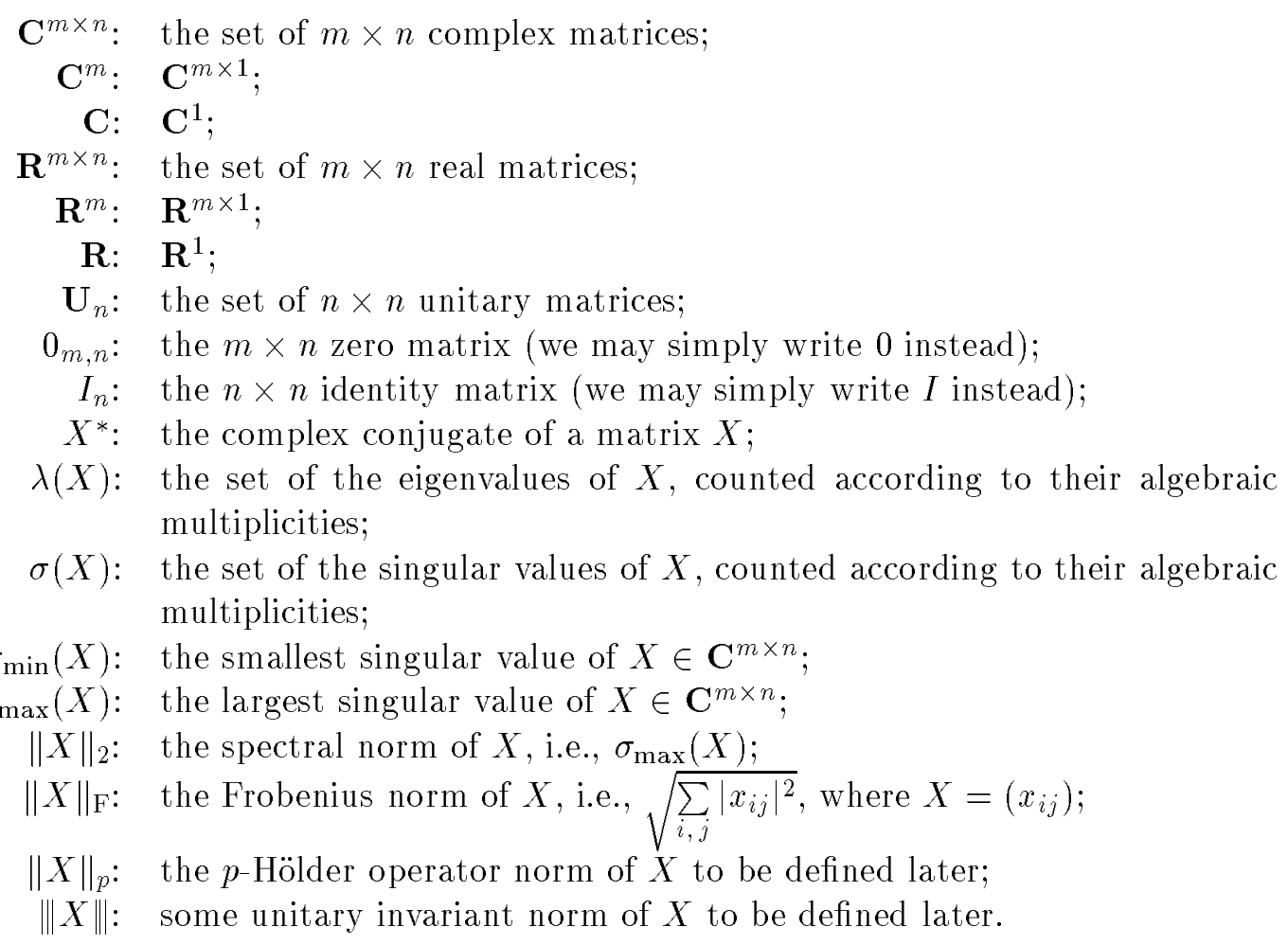




\subsection{Organization of the Paper}

We first in $\S 2$ summarize what we have accomplished in this paper, together with the corresponding well-known classical perturbation theorems that are being extended. In $\S 3$, we define two kinds of relative distances $\varrho_{p}(1 \leq p \leq \infty)$ and $\chi$ which will be heavily used in the rest of this paper. Various properties that are relevant to our relative perturbation theory are studied in the section and in Appendix A where it is proved $\varrho_{p}$ is indeed a metric on $\mathbf{R}$. Some of the classical perturbation theorems that will be extended to our relative perturbation theory are presented and briefly discussed in $\S 4$. We devote two sections to present and discuss main theorems of this paper: $\S 5$ is for theorems concerning nonnegative definite matrix eigenvalue variations and singular value variations; while $\S 6$ is for theorems concerning non-definite matrix eigenvalue variations. Proofs are postponed to later sections $\$ \S 8-10$. We discuss other developments in literature on relative perturbation theories in $\S 7$. We will touch how our relative perturbation theorems can be applied to generalized eigenvalue problems and generalized singular value problems in $\$ 11$. Finally, we present our conclusions.

\section{Summary of Results}

To help the reader to grasp quickly what we have accomplished in this paper, we give here a table to summarize partially the simplified (sometimes weakened) versions of our theorems in comparison with their corresponding well-known classical theorems in literature. Full statement of these theorems and their stronger versions will be given in $\S 5$ and $\S 6$. A theorem of Ostrowski in 1959 and more recent developments on the relative perturbation theory will be discussed in $\S 7$.

In what follows, we stick to the notation:

1. $A, \widetilde{A} \in \mathbf{C}^{n \times n}$ with eigenvalues

$$
\lambda(A)=\left\{\lambda_{1}, \cdots, \lambda_{n}\right\} \quad \text { and } \quad \lambda(\widetilde{A})=\left\{\tilde{\lambda}_{1}, \cdots, \tilde{\lambda}_{n}\right\} .
$$

Whenever, all $\lambda_{i}$ 's and $\tilde{\lambda}_{j}$ 's are real, we order them descendingly

$$
\lambda_{1} \geq \lambda_{2} \geq \cdots \geq \lambda_{n}, \quad \tilde{\lambda}_{1} \geq \tilde{\lambda}_{2} \geq \cdots \geq \tilde{\lambda}_{n}
$$

2. $B, \widetilde{B} \in \mathbf{C}^{m \times n}$ with singular values

$$
\sigma(B)=\left\{\sigma_{1}, \cdots, \sigma_{n}\right\} \text { and } \sigma(\widetilde{B})=\left\{\widetilde{\sigma}_{1}, \cdots, \widetilde{\sigma}_{n}\right\}
$$

ordered so that

$$
\sigma_{1} \geq \sigma_{2} \geq \cdots \geq \sigma_{n} \geq 0, \quad \widetilde{\sigma}_{1} \geq \widetilde{\sigma}_{2} \geq \cdots \geq \widetilde{\sigma}_{n} \geq 0
$$

In the table, $\tau$ always stands for some permutations of $\{1,2, \cdots, n\}$, and two relative distances $\varrho_{p}$ and $\chi$ are defined for $\alpha, \widetilde{\alpha} \in \mathbf{C}$ by

$$
\left.\varrho_{p}(\alpha, \tilde{\alpha})=\frac{|\alpha-\tilde{\alpha}|}{\sqrt[p]{|\alpha|^{p}+|\widetilde{\alpha}|^{p}}} \quad \text { for } 1 \leq p \leq \infty, \text { and } \quad \chi \alpha, \widetilde{\alpha}\right)=\frac{|\alpha-\tilde{\alpha}|}{\sqrt{|\alpha \widetilde{\alpha}|}},
$$

with convention $0 / 0=0$ for convenience. (For detailed studies of them, see $\S 3$.) 
Table 3.1. Perturbation Theorems for Eigenvalues

\begin{tabular}{|c|c|c|c|}
\hline \multicolumn{2}{|r|}{ Classical Bounds } & \multicolumn{2}{|r|}{ New Relative Bounds } \\
\hline $\begin{array}{l}A \\
\text { and } \\
\widetilde{A} \\
\text { Definite }\end{array}$ & $\begin{array}{l}\sqrt{\sum_{i=1}^{n}\left|\lambda_{i}-\widetilde{\lambda}_{i}\right|^{2}} \leq\|\widetilde{A}-A\|_{\mathrm{F}} \\
\text { (Theorems } 4.1 \text { and } 4.3 \text { ) }\end{array}$ & $\widetilde{A}=D^{*} A D$ & $\begin{array}{l}\sqrt{\sum_{i=1}^{n}\left[\chi\left(\lambda_{i}, \widetilde{\lambda}_{i}\right)\right]^{2}} \leq\left\|D^{*}-D^{-1}\right\|_{\mathrm{F}} \\
\text { (Theorem 5.1) }\end{array}$ \\
\hline $\begin{array}{l}A \\
\text { and } \\
\widetilde{A} \\
\text { Definite }\end{array}$ & $\begin{array}{l}\left|\lambda_{i}-\tilde{\lambda}_{i}\right| \leq\|\widetilde{A}-A\|_{2} \\
\text { (Theorem 4.3) }\end{array}$ & $\widetilde{A}=D^{*} A D$ & $\begin{array}{l}\chi\left(\lambda_{i}, \widetilde{\lambda}_{i}\right) \leq\left\|D^{*}-D^{-1}\right\|_{2} \\
\text { (Theorem 5.1) }\end{array}$ \\
\hline $\begin{array}{l}A \\
\text { and } \\
\widetilde{A} \\
\text { Hermitian }\end{array}$ & $\begin{array}{l}\sqrt{\sum_{i=1}^{n}\left|\lambda_{i}-\widetilde{\lambda}_{i}\right|^{2}} \leq\|\widetilde{A}-A\|_{\mathrm{F}} \\
\text { (Theorems } 4.1 \text { and } 4.3 \text { ) }\end{array}$ & $\widetilde{A}=D^{*} A D$ & $\begin{array}{l}\sqrt{\sum_{i=1}^{n}\left[\varrho_{2}\left(\lambda_{i}, \tilde{\lambda}_{\tau(i)}\right)\right]^{2}} \\
\quad \leq \sqrt{\|I-D\|_{\mathrm{F}}^{2}+\left\|I-D^{-1}\right\|_{\mathrm{F}}^{2}} \\
\text { (Theorem } 6.3 \text { ) }\end{array}$ \\
\hline $\begin{array}{l}A \\
\text { and } \\
\widetilde{A} \\
\text { Hermitian }\end{array}$ & $\begin{array}{l}\left|\lambda_{i}-\widetilde{\lambda}_{i}\right| \leq\|\widetilde{A}-A\|_{2} \\
\text { (Theorem 4.3) }\end{array}$ & $\widetilde{A}=D^{*} A D$ & $\begin{array}{l}\varrho_{\infty}\left(\lambda_{i}, \tilde{\lambda}_{i}\right) \leq\left\|I-D^{*} D\right\|_{2}, \\
\chi\left(\lambda_{i}, \widetilde{\lambda}_{i}\right) \leq \frac{\left\|I-D^{*} D\right\|_{2}}{\sigma_{\min }(D)} \\
(\text { see }(7.3) \text { and }(7.4))\end{array}$ \\
\hline $\begin{array}{l}A \\
\text { and } \\
\widetilde{A} \\
\text { normal }\end{array}$ & $\begin{array}{l}\sqrt{\sum_{i=1}^{n}\left|\lambda_{i}-\widetilde{\lambda}_{\tau(i)}\right|^{2}} \\
\leq\|\tilde{A}-A\|_{\mathrm{F}} \\
\text { (Theorem 4.1) }\end{array}$ & $\widetilde{A}=D_{1}^{*} A D_{2}$ & $\begin{array}{l}\sqrt{\sum_{i=1}^{n}\left[\varrho_{2}\left(\lambda_{i}, \tilde{\lambda}_{\tau(i)}\right)\right]^{2}} \leq \min \{ \\
\sqrt{\left\|I-D_{1}\right\|_{\mathrm{F}}^{2}+\left\|I-D_{2}^{-1}\right\|_{\mathrm{F}}^{2}}, \\
\left.\sqrt{\left\|I-D_{1}^{-1}\right\|_{\mathrm{F}}^{2}+\left\|I-D_{2}\right\|_{\mathrm{F}}^{2}}\right\} \\
\text { (Theorem } 6.2 \text { ) }\end{array}$ \\
\hline $\begin{array}{l}A=X \Lambda X^{-1} \\
\widetilde{A}=\widetilde{X} \widetilde{\Lambda} \widetilde{X}^{-1} \\
\Lambda, \widetilde{\Lambda} \text { may be } \\
\text { complex }\end{array}$ & $\begin{array}{l}\sqrt{\sum_{i=1}^{n}\left|\lambda_{i}-\widetilde{\lambda}_{\tau(i)}\right|^{2}} \\
\quad \leq \kappa(X) \kappa(\widetilde{X})\|\widetilde{A}-A\|_{\mathrm{F}} \\
\text { (Theorem 4.2) }\end{array}$ & $\widetilde{A}=D_{1}^{*} A D_{2}$ & 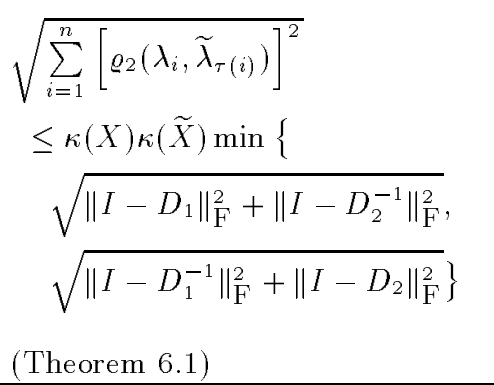 \\
\hline $\begin{array}{l}A=X \Lambda X^{-1} \\
\widetilde{A}=\widetilde{X} \widetilde{\Lambda} \widetilde{X}^{-1} \\
\Lambda \text { and } \widetilde{\Lambda} \text { real } \\
\text { nonnegative }\end{array}$ & $\begin{array}{l}\left|\lambda_{i}-\widetilde{\lambda}_{i}\right| \\
\quad \leq \sqrt{\kappa(X) \kappa(\tilde{X})}\|\widetilde{A}-A\|_{2} \\
\text { (Theorem 4.4) }\end{array}$ & $\widetilde{A}=D_{1}^{*} A D_{2}$ &  \\
\hline
\end{tabular}


Table 3.1 (continued). Perturbation Theorems for Singular Values

\begin{tabular}{|c|c|c|c|}
\hline \multicolumn{2}{|r|}{ Classical Bounds } & \multicolumn{2}{|r|}{ New Relative Bounds } \\
\hline $\begin{array}{l}B \\
\text { and } \\
\widetilde{B}\end{array}$ & $\begin{array}{l}\sqrt{\sum_{i=1}^{n}\left|\sigma_{i}-\widetilde{\sigma}_{i}\right|^{2}} \leq\|\widetilde{B}-B\|_{\mathrm{F}} \\
\text { (Theorem 4.7) }\end{array}$ & $\widetilde{B}=D_{1}^{*} B D_{2}$ & $\begin{array}{l}\sqrt{\sum_{i=1}^{n}\left[\chi\left(\sigma_{i}, \tilde{\sigma}_{\tau(i)}\right)\right]^{2}} \leq \\
\quad \frac{1}{2} \cdot \frac{\left\|D_{1}^{*}-D_{1}^{-1}\right\|_{\mathrm{F}}+\left\|D_{2}^{*}-D_{2}^{-1}\right\|_{\mathrm{F}}}{1-\frac{1}{32}\left\|D_{1}^{*}-D_{1}^{-1}\right\|_{2}\left\|D_{2}^{*}-D_{2}^{-1}\right\|_{2}} \\
\text { (Theorem 5.2) }\end{array}$ \\
\hline $\begin{array}{l}B \\
\text { and } \\
\widetilde{B}\end{array}$ & $\begin{array}{l}\sqrt{\sum_{i=1}^{n}\left|\sigma_{i}-\widetilde{\sigma}_{i}\right|^{2}} \leq\|\widetilde{B}-B\|_{\mathrm{F}} \\
\text { (Theorem 4.7) }\end{array}$ & $\widetilde{B}=D_{1}^{*} B D_{2}$ & $\begin{array}{l}\sqrt{\sum_{i=1}^{n}\left[\varrho_{p}\left(\sigma_{i}, \widetilde{\sigma}_{i}\right)\right]^{2}} \leq \frac{1}{2^{1+1 / p}} \times \\
\quad\left(\left\|D_{1}^{*}-D_{1}^{-1}\right\|_{\mathrm{F}}+\left\|D_{2}^{*}-D_{2}^{-1}\right\|_{\mathrm{F}}\right) \\
\text { (Theorem 5.3) }\end{array}$ \\
\hline $\begin{array}{l}B \\
\text { and } \\
\widetilde{B}\end{array}$ & $\begin{array}{l}\left|\sigma_{i}-\tilde{\sigma}_{i}\right| \leq\|\widetilde{B}-B\|_{2} \\
\text { (Theorem 4.7) }\end{array}$ & $\widetilde{B}=D_{1}^{*} B D_{2}$ & $\begin{array}{l}\chi\left(\sigma_{i}, \widetilde{\sigma}_{i}\right) \leq \\
\qquad \frac{1}{2} \cdot \frac{\left\|D_{1}^{*}-D_{1}^{-1}\right\|_{2}+\left\|D_{2}^{*}-D_{2}^{-1}\right\|_{2}}{1-\frac{1}{32}\left\|D_{1}^{*}-D_{1}^{-1}\right\|_{2}\left\|D_{2}^{*}-D_{2}^{-1}\right\|_{2}} \\
\text { (Theorem 5.2) }\end{array}$ \\
\hline $\begin{array}{l}B \\
\text { and } \\
\widetilde{B}\end{array}$ & $\begin{array}{l}\left|\sigma_{i}-\widetilde{\sigma}_{i}\right| \leq\|\widetilde{B}-B\|_{2} \\
\text { (Theorem 4.7) }\end{array}$ & $\widetilde{B}=D_{1}^{*} B D_{2}$ & $\begin{array}{l}\varrho_{p}\left(\sigma_{i}, \widetilde{\sigma}_{i}\right) \leq \frac{1}{2^{1+1 / p}} \times \\
\quad\left(\left\|D_{1}^{*}-D_{1}^{-1}\right\|_{2}+\left\|D_{2}^{*}-D_{2}^{-1}\right\|_{2}\right) \\
\text { (Theorem 5.3) }\end{array}$ \\
\hline
\end{tabular}

Table 3.1 (continued). A Bauer-Fike Type Theorem

\begin{tabular}{|c|c|c|c|}
\hline \multicolumn{2}{|r|}{ Classical Bounds } & \multicolumn{2}{|r|}{ New Relative Bounds } \\
\hline$A=X \Lambda X^{-1}$ & $\begin{array}{l}\forall \widetilde{\lambda} \in \lambda(\widetilde{A}), \exists \lambda \in \lambda(A) \text {, such } \\
\text { that } \\
\qquad|\widetilde{\lambda}-\lambda| \leq \kappa(X)\|\widetilde{A}-A\|_{2} \\
\text { (Theorem 4.6) }\end{array}$ & $\begin{array}{l}\text { Either } \\
\widetilde{A}=A D \\
\text { or } \\
\widetilde{A}=D A .\end{array}$ & $\begin{array}{l}\forall \widetilde{\lambda} \in \lambda(\widetilde{A}), \exists \lambda \in \lambda(A) \text {, such that } \\
\qquad \frac{|\widetilde{\lambda}-\lambda|}{|\lambda|} \leq \kappa(X)\|I-D\|_{2} \\
\text { (Theorem 6.6) }\end{array}$ \\
\hline
\end{tabular}

Finally, let's consider the graded cases:

1. $A=S^{*} H S$ and $\tilde{A}=S^{*} \tilde{H} S$ are two $n \times n$ graded nonnegative definite Hermitian matrices. $H$ is nonsingular and $\left\|H^{-1}\right\|_{2}\|\Delta H\|_{2}<1$, where $\Delta H \stackrel{\text { def }}{=} \widetilde{H}-H$.

2. $B=G S$ and $\widetilde{B}=\widetilde{G} S$ are two $m \geq n$ graded matrices whose singular values are of interest. $G$ is nonsingular and $\left\|G^{-1}\right\|_{2}\|\Delta G\|_{2}<1$, where $\Delta G \stackrel{\text { def }}{=} \widetilde{G}-G$.

In applications, $S$ is scaling matrices and often are diagonal; but our results do not assume this. The elements of $S$ can vary wildly. The interesting case is when $H(G)$ is much better conditioned than $A(B)$, and when $\Delta H(\Delta G)$ is small even though $A-\widetilde{A}(B-\widetilde{B})$ is not. 
Table 3.1 (continued). Perturbation Theorems for Graded Matrices

\begin{tabular}{|c|c|c|c|}
\hline \multicolumn{2}{|r|}{ Classical Bounds } & \multicolumn{2}{|r|}{ New Relative Bounds } \\
\hline $\begin{array}{l}A \\
\text { and } \\
\widetilde{A} \\
\text { Definite }\end{array}$ & $\begin{array}{l}\sqrt{\sum_{i=1}^{n}\left|\lambda_{i}-\widetilde{\lambda}_{i}\right|^{2}} \leq\|\widetilde{A}-A\|_{\mathrm{F}} \\
\text { (Theorems } 4.1 \text { and } 4.3 \text { ) }\end{array}$ & $\begin{array}{l}A=S^{*} H S \\
\widetilde{A}=S^{*} \widetilde{H} S\end{array}$ & $\begin{array}{l}\sqrt{\sum_{i=1}^{n}\left[\chi\left(\lambda_{i}, \widetilde{\lambda}_{i}\right)\right]^{2}} \\
\quad \leq \frac{\left\|H^{-1}\right\|_{2}\|\Delta H\|_{\mathrm{F}}}{\sqrt{1-\left\|H^{-1}\right\|_{2}\|\Delta H\|_{2}}} \\
\text { (Theorem 5.4) }\end{array}$ \\
\hline $\begin{array}{l}A \\
\text { and } \\
\widetilde{A} \\
\text { Definite }\end{array}$ & $\begin{array}{l}\left|\lambda_{i}-\widetilde{\lambda}_{i}\right| \leq\|\widetilde{A}-A\|_{2} \\
\text { (Theorem 4.3) }\end{array}$ & $\begin{array}{l}A=S^{*} H S \\
\widetilde{A}=S^{*} \widetilde{H} S\end{array}$ & $\begin{array}{l}\chi\left(\lambda_{i}, \widetilde{\lambda}_{i}\right) \leq \frac{\left\|H^{-1}\right\|_{2}\|\Delta H\|_{2}}{\sqrt{1-\left\|H^{-1}\right\|_{2}\|\Delta H\|_{2}}} \\
\text { (Theorem 5.4) }\end{array}$ \\
\hline $\begin{array}{l}B \\
\text { and } \\
\widetilde{B}\end{array}$ & $\begin{array}{l}\sqrt{\sum_{i=1}^{n}\left|\sigma_{i}-\widetilde{\sigma}_{i}\right|^{2}} \leq\|\widetilde{B}-B\|_{\mathrm{F}} \\
\text { (Theorem 4.7) }\end{array}$ & $\begin{array}{l}B=G S \\
\text { and } \\
\widetilde{B}=\widetilde{G} S\end{array}$ & $\begin{array}{l}\sqrt{\sum_{i=1}^{n}\left[\chi\left(\sigma_{i}, \tilde{\sigma}_{i}\right)\right]^{2}} \leq \\
\frac{2-\left\|G^{-1}\right\|_{2}\|\Delta G\|_{2}}{2\left(1-\left\|G^{-1}\right\|_{2}\|\Delta G\|_{2}\right)}\left\|G^{-1}\right\|_{2}\|\Delta G\|_{\mathrm{F}} \\
\text { (Theorem 5.5) }\end{array}$ \\
\hline $\begin{array}{l}B \\
\text { and } \\
\widetilde{B}\end{array}$ & $\begin{array}{l}\left|\sigma_{i}-\widetilde{\sigma}_{i}\right|^{2} \leq\|\widetilde{B}-B\|_{2} \\
\text { (Theorem 4.7) }\end{array}$ & $\begin{array}{l}B=G S \\
\text { and } \\
\widetilde{B}=\widetilde{G} S\end{array}$ & $\begin{array}{l}\chi\left(\sigma_{i}, \tilde{\sigma}_{i}\right) \leq \\
\quad \frac{2-\left\|G^{-1}\right\|_{2}\|\Delta G\|_{2}}{2\left(1-\left\|G^{-1}\right\|_{2}\|\Delta G\|_{2}\right)}\left\|G^{-1}\right\|_{2}\|\Delta G\|_{2} \\
\text { (Theorem 5.5) }\end{array}$ \\
\hline
\end{tabular}

\section{Relative Distances}

This section is devoted to studying two different kinds of relative distances measuring relative errors between two (complex) numbers $\alpha$ and $\widetilde{\alpha}$ one of which is an approximation of the other. Classically, the relative error in $\widetilde{\alpha}=\alpha(1+\delta)$ as an approximation to $\alpha$ is measured by

$$
\delta=\text { relative error in } \widetilde{\alpha}=\frac{\widetilde{\alpha}-\alpha}{\alpha} \text {. }
$$

When $|\delta| \leq \epsilon$ we say that the relative perturbation to $\alpha$ is at most $\epsilon$ (see, e.g., [8]). Such an measurement lacks mathematical properties upon which a nice relative perturbation theory can be bulit: for example, it lacks symmetry between $\alpha$ and $\widetilde{\alpha}$ and thus it can not be a metric among spaces of numbers that are of interest to us. Nonetheless, it is good enough for measuring correct digits of numerical approximations.

In what follows, new relative distances will be proposed and studied. These distances have better mathematical properties that allow us to develop a nice perturbation theory and yet they are topologically equivalent to the classical measurement $|\delta|$ as defined in (3.1). 


\subsection{The $p$-Relative Distance}

The $p$-relative distance between $\alpha, \widetilde{\alpha} \in \mathbf{C}$ is defined as

$$
\varrho_{p}(\alpha, \tilde{\alpha}) \stackrel{\text { def }}{=} \frac{|\alpha-\widetilde{\alpha}|}{\sqrt[p]{|\alpha|^{p}+|\widetilde{\alpha}|^{p}}} \text { for } 1 \leq p \leq \infty .
$$

We define, for convenience, $0 / 0 \stackrel{\text { def }}{=} 0$. $\varrho_{\infty}$ was first used by Deift, Demmel, Li, and Tomei $[6$, 1991] to define relative gaps.

Proposition 3.1 Let $1 \leq p \leq \infty$ and $\alpha, \widetilde{\alpha} \in \mathbf{C}$.

1. $\varrho_{p}(\alpha, \widetilde{\alpha}) \geq 0$; and $\varrho_{p}(\alpha, \widetilde{\alpha})=0$ if and only if $\alpha=\widetilde{\alpha}$.

2. $\varrho_{p}(\alpha, \widetilde{\alpha})=\varrho_{p}(\widetilde{\alpha}, \alpha)$.

3. $\varrho_{p}(\xi \alpha, \xi \widetilde{\alpha})=\varrho_{p}(\alpha, \widetilde{\alpha})$ for all $0 \neq \xi \in \mathbf{C}$.

4. $\varrho_{p}(1 / \alpha, 1 / \widetilde{\alpha})=\varrho_{p}(\alpha, \tilde{\alpha})$ for $\alpha \neq 0$ and $\tilde{\alpha} \neq 0$.

5. $\varrho_{p}(\alpha, \widetilde{\alpha}) \leq 2^{1-1 / p} ;$ and $\varrho_{p}(\alpha, \widetilde{\alpha})=2^{1-1 / p}$ if and only if $\alpha=-\widetilde{\alpha} \neq 0$ in the case $p>1 ; \varrho_{1}(\alpha, \widetilde{\alpha})=1$ if and only if $\alpha \widetilde{\alpha} \leq 0$ and at least one of $\alpha$ and $\widetilde{\alpha}$ is not zero.

6. $\varrho_{p}(\alpha, 0) \equiv 1$ if $\alpha \neq 0$; and

$$
\varrho_{p}(\alpha, \tilde{\alpha})\left\{\begin{array}{l}
>1, \text { for } p>1 \text { and } \alpha \tilde{\alpha}<0 \\
<1, \text { for all } p \geq 1 \text { and } \alpha \tilde{\alpha}>0 .
\end{array}\right.
$$

7. $\varrho_{p}(\alpha, \widetilde{\alpha})$ increases as $p$ does, and

$$
\varrho_{p}(\alpha, \widetilde{\alpha}) \leq \varrho_{2 p}(\alpha, \widetilde{\alpha}) \leq 2^{1 /(2 p)} \varrho_{p}(\alpha, \widetilde{\alpha}) .
$$

8. if $\alpha, \beta, \widetilde{\alpha}, \widetilde{\beta} \in \mathbf{R}$ and $\alpha \leq \beta \leq \widetilde{\beta} \leq \widetilde{\alpha}$, then

$$
\varrho_{1}(\alpha, \tilde{\alpha}) \geq \varrho_{1}(\beta, \tilde{\beta})
$$

if, in addition to the listed conditions, also $\beta \widetilde{\beta} \geq 0$, then

$$
\varrho_{p}(\alpha, \widetilde{\alpha}) \geq \varrho_{p}(\beta, \widetilde{\beta}) \quad \text { for } p>1,
$$

and inequality (3.3) is strict if either $\alpha<\beta$ or $\widetilde{\beta}<\widetilde{\alpha}$ holds.

Proof: Properties 1-6 are easy to verify. Property 7 holds because $\sqrt[p]{|\alpha|^{p}+|\widetilde{\alpha}|^{p}}$ is a decreasing function of $p$ for $1 \leq p \leq \infty$, and

$$
\left(|\alpha|^{p}+|\widetilde{\alpha}|^{p}\right)^{1 / p} \leq\left(\sqrt{2} \sqrt{|\alpha|^{2 p}+|\widetilde{\alpha}|^{2 p}}\right)^{1 / p}=2^{1 /(2 p)}\left(|\alpha|^{2 p}+|\widetilde{\alpha}|^{2 p}\right)^{1 /(2 p)}
$$


by the Cauchy-Schwarz inequality ${ }^{1}$. To prove Property 8 , we consider function $f(\xi)$ defined by

$$
f(\xi) \stackrel{\text { def }}{=} \frac{1-\xi}{\sqrt[p]{1+|\xi|^{p}}}, \quad \text { where }-1 \leq \xi \leq 1
$$

When $p=1$,

$$
f(\xi)= \begin{cases}1, & \text { for }-1 \leq \xi \leq 0, \\ \frac{2}{1+\xi}-1, & \text { for } 0 \leq \xi \leq 1\end{cases}
$$

so $f(\xi)$ decreases monotonically and decreases strictly monotonically for $0 \leq \xi \leq 1$. We are about to prove that when $p>1$ function $f(\xi)$ so defined is strictly monotonically decreasing. This is true if $p=\infty$. When $1<p<\infty$, set $h(\xi) \stackrel{\text { def }}{=}[f(\xi)]^{p}$ and $g(\xi) \stackrel{\text { def }}{=}$ $[f(-\xi)]^{p}$. Because for $0<\xi<1$

$$
h^{\prime}(\xi)=-\frac{p(1-\xi)^{p-1}\left(1+\xi^{p-1}\right)}{\left(1+\xi^{p}\right)^{2}}<0 \quad \text { and } \quad g^{\prime}(\xi)=\frac{p(1+\xi)^{p-1}\left(1-\xi^{p-1}\right)}{\left(1+\xi^{p}\right)^{2}}>0,
$$

for $0<\xi<1, h(\xi)$ is strictly monotonically decreasing, and $g(\xi)$ is strictly monotonically increasing. Thus function $f(\xi)$ is strictly monotonically decreasing for $p>1$.

There are several cases to deal with for confirming Property 8 . Assume at least one of $\alpha \leq \beta$ and $\widetilde{\beta} \leq \widetilde{\alpha}$ is strict.

1. $0 \leq \alpha \leq \beta \leq \widetilde{\beta} \leq \widetilde{\alpha}$, then $0 \leq \alpha / \widetilde{\alpha}<\beta / \widetilde{\beta} \leq 1$; thus

$$
\varrho_{p}(\alpha, \widetilde{\alpha})=f(\alpha / \widetilde{\alpha})>f(\beta / \widetilde{\beta})=\varrho_{p}(\beta, \widetilde{\beta}) .
$$

2. $\alpha \leq 0 \leq \beta \leq \widetilde{\beta} \leq \widetilde{\alpha}$ or $\alpha \leq \beta \leq \widetilde{\beta} \leq 0 \leq \widetilde{\alpha}$, then Property 6 implies

$$
\varrho_{p}(\alpha, \widetilde{\alpha}) \geq 1 \geq \varrho_{p}(\beta, \widetilde{\beta})
$$

It is easy to verify that the equalities in the two " $\geq$ 's" can not be satisfied simutaneously.

3. $\alpha \leq \beta \leq 0 \leq \widetilde{\beta} \leq \widetilde{\alpha}$. Only $p=1$ shall be considered:

$$
\varrho_{1}(\alpha, \widetilde{\alpha})=1 \geq 1=\varrho_{1}(\beta, \widetilde{\beta}) .
$$

4. $\alpha \leq \beta \leq \widetilde{\beta} \leq \widetilde{\alpha} \leq 0$, then $0 \leq \widetilde{\alpha} / \alpha<\widetilde{\beta} / \beta \leq 1$; thus

$$
\varrho_{p}(\alpha, \widetilde{\alpha})=f(\widetilde{\alpha} / \alpha)>f(\widetilde{\beta} / \beta)=\varrho_{p}(\beta, \widetilde{\beta}) .
$$

${ }^{1}$ Hölder inequality: For $\alpha, \widetilde{\alpha}, \beta, \widetilde{\beta} \geq 0$, and $1 \leq p \leq \infty$,

$$
\alpha \beta+\tilde{\alpha} \widetilde{\beta} \leq \sqrt[p]{\alpha^{p}+\widetilde{\alpha}^{p}} \sqrt[q]{\beta^{q}+\widetilde{\beta}^{q}}
$$

and the equality holds if and only if $\alpha^{p} \widetilde{\beta}^{q}=\widetilde{\alpha}^{p} \beta^{q}$, where $q=p /(p-1)$. When $p=2$, this is the Cauchy-Schwarz inequality. The Hölder inequality will be used frequently later in our proofs. 
The proof of Property 8 is completed.

Remark: In Property 8 of Proposition 3.1, assumption $\beta \widetilde{\beta} \geq 0$ for the case $p>1$ is essential. A counterexample is: let $\xi>\zeta>0$, and let $\alpha=-\zeta \leq \beta=-\zeta<\widetilde{\beta}=\zeta<\widetilde{\alpha}<\xi$. Then

$$
\varrho_{p}(\alpha, \widetilde{\alpha})=\frac{\xi+\zeta}{\sqrt[p]{\xi^{p}+\zeta^{p}}}<2^{1-1 / p}=\varrho_{p}(\beta, \widetilde{\beta}) .
$$

The following proposition establishes the topological equivalence between the classical measurement (see (3.1)) and our new relative distances $\varrho_{p}$.

Proposition 3.2 Let $0 \leq \epsilon<1$, and $\alpha, \widetilde{\alpha} \in \mathbf{R}$. We have the following:

$$
\left|\frac{\widetilde{\alpha}}{\alpha}-1\right| \leq \epsilon \Rightarrow \varrho_{p}(\alpha, \widetilde{\alpha}) \leq \frac{\epsilon}{\sqrt[p]{1+(1-\epsilon)^{p}}}
$$

and

$$
\varrho_{p}(\alpha, \widetilde{\alpha}) \leq \epsilon \Rightarrow \max \left\{\left|\frac{\widetilde{\alpha}}{\alpha}-1\right|,\left|\frac{\alpha}{\widetilde{\alpha}}-1\right|\right\} \leq \frac{2^{1 / p} \epsilon}{1-\epsilon}
$$

Asymptotically,

$$
\lim _{\widetilde{\alpha} \rightarrow \alpha} \frac{\varrho_{p}(\alpha, \widetilde{\alpha})}{\left|\frac{\widetilde{\alpha}}{\alpha}-1\right|}=2^{1 / p},
$$

thus (3.4) and (3.5) are at least asymptotically sharp.

Proof: $\left|\frac{\tilde{\alpha}}{\alpha}-1\right| \leq \epsilon$ implies $|\tilde{\alpha} / \alpha| \geq 1-\epsilon$; so

$$
\varrho_{p}(\alpha, \tilde{\alpha})=\left|\frac{\tilde{\alpha}-\alpha}{\alpha}\right| \cdot \frac{1}{\sqrt[p]{1+|\tilde{\alpha} / \alpha|^{p}}} \leq \frac{\epsilon}{\sqrt[p]{1+(1-\epsilon)^{p}}} .
$$

This confirms (3.4). (3.5) $)_{p=1}$ and $(3.5)_{p=\infty}$ can be proved analogously to what we are going to do for $1<p<\infty$. Let $\xi=\widetilde{\alpha} / \alpha$ or $\xi=\alpha / \widetilde{\alpha}$. Then $\epsilon<1 \Rightarrow \xi>0$ by Proposition 3.1. $\varrho_{p}(\alpha, \widetilde{\alpha}) \leq \epsilon$ implies

$$
\eta \stackrel{\text { def }}{=} \frac{|\xi-1|}{\sqrt[n]{1+\xi^{p}}} \leq \epsilon .
$$

Now if $\xi<1$, then $|\xi-1| \leq \epsilon \sqrt[p]{1+\xi^{p}} \leq 2^{1 / p} \epsilon$. Assume $\xi>1$ and write $\zeta=\xi-1>0$. (3.6) yields $\zeta=\eta \sqrt[p]{1+(1+\zeta)^{p}}$; and thus

$$
\eta^{p}(1+\zeta)^{p}-\zeta^{p}+\eta^{p}=0
$$

Consider function $f(x)=\eta^{p}(1+x)^{p}-x^{p}+\eta^{p}$ for $x \geq 0$. It is easy to see that

$$
f(0)=0, f^{\prime}(0)=p \eta^{p}>0, \text { and } f(+\infty)<0,
$$

and $f^{\prime}(x)$ vanishes only once at $x=\eta^{q} /\left(1-\eta^{q}\right)$, where $q=p /(p-1)$; So $f(x)$ has a unique positive zero which is $\zeta$. Now if we can show $f\left(\frac{2^{1 / p} \eta}{1-\eta}\right) \leq 0$, then $\zeta \leq \frac{2^{1 / p} \eta}{1-\eta}$ must be true; and then by $\eta \leq \epsilon$

$$
\zeta \leq \frac{2^{1 / p} \eta}{1-\eta} \leq \frac{2^{1 / p} \epsilon}{1-\epsilon}
$$


as was to be shown. We have to prove $f\left(\frac{2^{1 / p} \eta}{1-\eta}\right) \leq 0$. This is equivalent to

$$
\left(1+\left(2^{1 / p}-1\right) \eta\right)^{p}+(1-\eta)^{p}-2 \leq 0
$$

Consider function $g(x)=\left(1+\left(2^{1 / p}-1\right) x\right)^{p}+(1-x)^{p}-2$ for $0 \leq x \leq 1$. It is easy to see that

$$
g(0)=g(1)=0, g^{\prime}(0)=-p\left(2-2^{1 / p}\right)<0, g^{\prime}(1)=p\left(2-2^{1 / q}\right)>0,
$$

and $g^{\prime}(x)$ vanishes only once for $x \geq 0$; so must $g(x) \leq 0$ for $0 \leq x \leq 1$.

Proposition 3.3 Let $\tilde{\alpha}=\alpha\left(1+\delta_{1}\right) \in \mathbf{C}$ and $\widetilde{\beta}=\beta\left(1+\delta_{2}\right) \in \mathbf{C}$. If $\left|\delta_{i}\right| \leq \epsilon<1$, then

$$
\begin{aligned}
& \frac{\varrho_{p}(\alpha, \beta)}{1-\epsilon}+\frac{\epsilon}{1-\epsilon} \geq \varrho_{p}(\alpha, \widetilde{\beta}) \geq \frac{\varrho_{p}(\alpha, \beta)}{1+\epsilon}-\frac{\epsilon}{1+\epsilon}, \\
& \frac{\varrho_{p}(\alpha, \beta)}{1-\epsilon}+\frac{2^{1 / q} \epsilon}{1-\epsilon} \geq \varrho_{p}(\widetilde{\alpha}, \widetilde{\beta}) \geq \frac{\varrho_{p}(\alpha, \beta)}{1+\epsilon}-\frac{2^{1 / q} \epsilon}{1+\epsilon},
\end{aligned}
$$

where $q=p /(p-1)$.

Proof: We will only provide a proof of (3.8) since the proof of (3.7) is analogous. Notice that

$$
|\alpha|(1-\epsilon) \leq|\widetilde{\alpha}| \leq|\alpha|(1+\epsilon) \quad \text { and } \quad|\beta|(1-\epsilon) \leq|\widetilde{\beta}| \leq|\beta|(1+\epsilon)
$$

so

$$
\begin{aligned}
& \varrho_{p}(\widetilde{\alpha}, \widetilde{\beta})=\frac{|\widetilde{\alpha}-\widetilde{\beta}|}{\sqrt[p]{|\widetilde{\alpha}|^{p}+|\widetilde{\beta}|^{p}}} \geq \frac{|\alpha-\beta|-\left|\alpha \delta_{1}-\beta \delta_{2}\right|}{\sqrt[p]{|\alpha|^{p}+|\beta|^{p}}(1+\epsilon)} \\
& \geq \frac{|\alpha-\beta|-\sqrt[p]{|\alpha|^{p}+|\beta|^{p}} \sqrt[q]{\epsilon^{q}+\epsilon^{q}}}{\sqrt[p]{|\alpha|^{p}+|\beta|^{p}}(1+\epsilon)} \\
& =\frac{\varrho_{p}(\alpha, \beta)}{1+\epsilon}-\frac{2^{1 / q_{\epsilon}} \epsilon}{1+\epsilon}, \\
& \varrho_{p}(\widetilde{\alpha}, \widetilde{\beta}) \leq \frac{|\alpha-\beta|+\left|\alpha \delta_{1}-\beta \delta_{2}\right|}{\sqrt[p]{|\alpha|^{p}+|\beta|^{p}}(1-\epsilon)} \\
& \leq \frac{|\alpha-\beta|+\sqrt[p]{|\alpha|^{p}+|\beta|^{p}} \sqrt[q]{\epsilon^{q}+\epsilon^{q}}}{\sqrt[p]{|\alpha|^{p}+|\beta|^{p}}(1-\epsilon)} \\
& =\frac{\varrho_{p}(\alpha, \beta)}{1-\epsilon}+\frac{2^{1 / q} \epsilon}{1-\epsilon},
\end{aligned}
$$

as were to be shown.

Proposition 3.4 For $\alpha, \widetilde{\alpha} \in \mathbf{C}$ and $1 \leq p \leq \infty$, we have

$$
\left[2^{1-1 /(2 p)}-\varrho_{2 p}(\alpha, \widetilde{\alpha})\right] \varrho_{2 p}(\alpha, \widetilde{\alpha}) \leq \varrho_{p}\left(\alpha^{2}, \widetilde{\alpha}^{2}\right) \leq 2^{1-1 /(2 p)} \varrho_{2 p}(\alpha, \widetilde{\alpha}) \leq 2 \varrho_{p}(\alpha, \widetilde{\alpha}) ;
$$

for $\alpha, \tilde{\alpha} \in \mathbf{R}$ and $\alpha \tilde{\alpha} \geq 0$, we have

$$
\varrho_{p}(\alpha, \widetilde{\alpha}) \leq \varrho_{2 p}(\alpha, \widetilde{\alpha}) \leq \varrho_{p}\left(\alpha^{2}, \widetilde{\alpha}^{2}\right) .
$$


Proof: No proof is necessary if $\alpha=\widetilde{\alpha}=0$. Assume at least one of $\alpha$ and $\widetilde{\alpha}$ is not zero. Notice that

$$
\begin{aligned}
\varrho_{p}\left(\alpha^{2}, \widetilde{\alpha}^{2}\right) & =\frac{\left|\alpha^{2}-\tilde{\alpha}^{2}\right|}{\left(|\alpha|^{2 p}+|\widetilde{\alpha}|^{2 p}\right)^{1 / p}}=\frac{|\alpha+\widetilde{\alpha}|}{\left(|\alpha|^{2 p}+|\widetilde{\alpha}|^{2 p}\right)^{1 /(2 p)}} \times \frac{|\alpha-\tilde{\alpha}|}{\left(|\alpha|^{2 p}+|\widetilde{\alpha}|^{2 p}\right)^{1 /(2 p)}} \\
& =\frac{|\alpha+\widetilde{\alpha}|}{\left(|\alpha|^{2 p}+|\widetilde{\alpha}|^{2 p}\right)^{1 /(2 p)}} \varrho_{2 p}(\alpha, \widetilde{\alpha}) .
\end{aligned}
$$

and that $|\alpha+\widetilde{\alpha}| \leq 2^{1-1 /(2 p)}\left(|\alpha|^{2 p}+|\widetilde{\alpha}|^{2 p}\right)^{1 /(2 p)}$. Then

$$
\varrho_{p}\left(\alpha^{2}, \widetilde{\alpha}^{2}\right) \leq 2^{1-1 /(2 p)} \varrho_{2 p}(\alpha, \widetilde{\alpha}) \leq 2 \varrho_{p}(\alpha, \widetilde{\alpha})
$$

by Property 7 of Proposition 3.1. To complete the proof of (3.9), we also notice that (without loss of generality, assuming $|\alpha| \geq|\widetilde{\alpha}|$.)

$$
\begin{aligned}
\frac{|\alpha+\widetilde{\alpha}|}{\left(|\alpha|^{2 p}+|\widetilde{\alpha}|^{2 p}\right)^{1 /(2 p)}} & =\frac{|2 \alpha-(\alpha-\widetilde{\alpha})|}{\left(|\alpha|^{2 p}+|\widetilde{\alpha}|^{2 p}\right)^{1 /(2 p)}} \geq \frac{|2 \alpha|}{\left(|\alpha|^{2 p}+|\widetilde{\alpha}|^{2 p}\right)^{1 /(2 p)}}-\frac{|\alpha-\widetilde{\alpha}|}{\left(|\alpha|^{2 p}+|\widetilde{\alpha}|^{2 p}\right)^{1 /(2 p)}} \\
& =\frac{2}{\left(1+\xi^{2 p}\right)^{1 /(2 p)}}-\varrho_{2 p}(\alpha, \widetilde{\alpha}) \geq 2^{1-1 /(2 p)}-\varrho_{2 p}(\alpha, \widetilde{\alpha}),
\end{aligned}
$$

where $0 \leq \xi=|\widetilde{\alpha} / \alpha| \leq 1$. To prove (3.10), we see under the condition $\alpha \tilde{\alpha} \geq 0$ that $|\alpha+\widetilde{\alpha}|=|\alpha|+|\widetilde{\alpha}| \geq\left(|\alpha|^{2 p}+|\widetilde{\alpha}|^{2 p}\right)^{1 /(2 p)}$.

Let $\left\{\alpha_{1}, \alpha_{2}, \cdots, \alpha_{n}\right\}$ and $\left\{\tilde{\alpha}_{1}, \tilde{\alpha}_{2}, \cdots, \widetilde{\alpha}_{n}\right\}$ be two sequences of $n$ real numbers ordered ascendingly ${ }^{2}$, i.e.,

$$
\alpha_{1} \leq \alpha_{2} \leq \cdots \leq \alpha_{n}, \quad \widetilde{\alpha}_{1} \leq \widetilde{\alpha}_{2} \leq \cdots \leq \widetilde{\alpha}_{n}
$$

Now we address the following question: What are the best one-one pairings between the $\alpha_{i}$ 's and the $\widetilde{\alpha}_{j}$ 's under certain measures?. Such a question will become important later in this paper when we try to pair the eigenvalues of one matrix to these of another.

\section{Proposition 3.5}

$$
\max _{1 \leq i \leq n} \varrho_{1}\left(\alpha_{i}, \widetilde{\alpha}_{i}\right)=\min _{\tau} \max _{1 \leq i \leq n} \varrho_{1}\left(\alpha_{i}, \widetilde{\alpha}_{\tau(i)}\right)
$$

and for $p>1$ if all $\alpha_{i}$ 's and $\widetilde{\alpha}_{j}$ 's are nonnegative,

$$
\max _{1 \leq i \leq n} \varrho_{p}\left(\alpha_{i}, \widetilde{\alpha}_{i}\right)=\min _{\tau} \max _{1 \leq i \leq n} \varrho_{p}\left(\alpha_{i}, \widetilde{\alpha}_{\tau(i)}\right)
$$

Here the minimizations are taken over all permutations $\tau$ of $\{1,2, \cdots, n\}$.

Proof: For any permutation $\tau$ of $\{1,2, \cdots, n\}$, the idea of our proof is to construct $n+1$ permutations $\tau_{j}$ such that

$$
\tau_{0}=\tau, \quad \tau_{n}=\text { identity permutation }
$$

\footnotetext{
${ }^{2}$ The situation when they are ordered descendingly can be handled in exactly the same way.
} 
and for $j=0,1,2, \cdots, n-1$

$$
\max _{1 \leq i \leq n} \varrho_{p}\left(\alpha_{i}, \widetilde{\alpha}_{\tau_{j}(i)}\right) \geq \max _{1 \leq i \leq n} \varrho_{p}\left(\alpha_{i}, \widetilde{\alpha}_{\tau_{j+1}(i)}\right) .
$$

The construction of these $\tau_{j}$ 's goes as follows: Set $\tau_{0}=\tau$. Given $\tau_{j}$, if $\tau_{j}(j+1)=j+1$, set $\tau_{j+1}=\tau_{j}$; otherwise define

$$
\tau_{j+1}(i)= \begin{cases}\tau_{j}(i), & \text { for } \tau_{j}^{-1}(j+1) \neq i \neq j+1, \\ j+1, & \text { for } i=j+1 \\ \tau_{j}(j+1), & \text { for } i=\tau_{j}^{-1}(j+1) .\end{cases}
$$

In this latter case, $\tau_{j}$ and $\tau_{j+1}$ differ only at two indexs as shown in the following picture (notice that $\tau_{j}^{-1}(j+1)>j+1$ and $\tau_{j}(j+1)>j+1$ ):

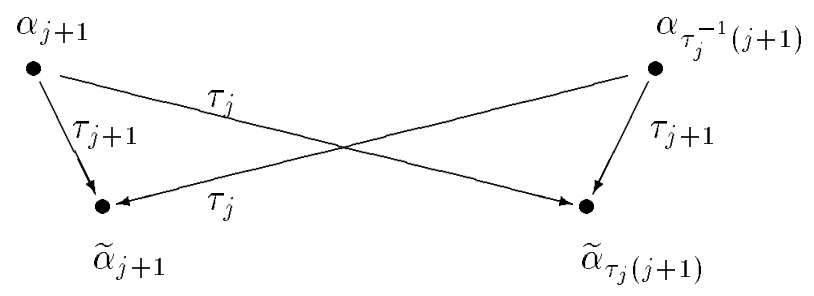

With Property 8 in Proposition 3.1, it is easy to prove that

$$
\begin{aligned}
& \max \left\{\varrho_{p}\left(\alpha_{j+1}, \widetilde{\alpha}_{\tau_{j}(j+1)}\right), \varrho_{p}\left(\alpha_{\tau_{j}^{-1}(j+1)}, \widetilde{\alpha}_{j+1}\right)\right\} \\
& \quad \geq \max \left\{\varrho_{p}\left(\alpha_{j+1}, \widetilde{\alpha}_{j+1}\right), \varrho_{p}\left(\alpha_{\tau_{j}^{-1}(j+1)}, \widetilde{\alpha}_{\tau_{j}(j+1)}\right)\right\} .
\end{aligned}
$$

Thus $\tau_{j}$ 's so constructed have the desired properties.

Remark. Proposition 3.5 may fail if not all of the $\alpha_{i}$ 's and $\widetilde{\alpha}_{j}$ 's are of the same sign in the case $p>1$. A counterexample is as follows: $n=2$ and

$$
\alpha_{1}=-2<\alpha_{2}=1 \quad \text { and } \quad \widetilde{\alpha}_{1}=2<\widetilde{\alpha}_{2}=4 .
$$

Then (see Proposition 3.1)

$$
\begin{aligned}
\max & \left\{\varrho_{p}\left(\alpha_{1}, \widetilde{\alpha}_{1}\right), \varrho_{p}\left(\alpha_{2}, \widetilde{\alpha}_{2}\right)\right\}=\varrho_{p}\left(\alpha_{1}, \widetilde{\alpha}_{1}\right)=2^{1-1 / p} \\
& >\frac{6}{\sqrt[p]{2^{p}+4^{p}}}=\varrho_{p}\left(\alpha_{1}, \widetilde{\alpha}_{2}\right)=\max \left\{\varrho_{p}\left(\alpha_{1}, \widetilde{\alpha}_{2}\right), \varrho_{p}\left(\alpha_{2}, \widetilde{\alpha}_{1}\right)\right\} .
\end{aligned}
$$

Another point we want to make is that given two sequences of $\alpha_{i}$ 's and $\tilde{\alpha}_{j}$ 's ordered as in (3.11), generally

$$
\sum_{i=1}^{n}\left[\varrho_{p}\left(\alpha_{i}, \widetilde{\alpha}_{i}\right)\right]^{2} \neq \min _{\tau} \sum_{i=1}^{n}\left[\varrho_{p}\left(\alpha_{i}, \widetilde{\alpha}_{\tau(i)}\right)\right]^{2},
$$

even if all $\alpha_{i}, \widetilde{\alpha}_{j}>0$. Here is a counterexample: $n=2$

$$
0<\alpha_{1}<\widetilde{\alpha}_{1}<\alpha_{2}=\widetilde{\alpha}_{2} / 2<\widetilde{\alpha}_{2}
$$


where $\alpha_{1}$ is sufficiently close to 0 , and $\tilde{\alpha}_{1}$ is sufficiently close to $\alpha_{2}$ which is fixed. Since as $\alpha_{1} \rightarrow 0^{+}$and $\widetilde{\alpha}_{1} \rightarrow \alpha_{2}^{-}$

$$
\begin{aligned}
& {\left[\varrho_{p}\left(\alpha_{1}, \widetilde{\alpha}_{2}\right)\right]^{2}+\left[\varrho_{p}\left(\alpha_{2}, \widetilde{\alpha}_{1}\right)\right]^{2} \rightarrow 1} \\
& {\left[\varrho_{p}\left(\alpha_{1}, \widetilde{\alpha}_{1}\right)\right]^{2}+\left[\varrho_{p}\left(\alpha_{2}, \widetilde{\alpha}_{2}\right)\right]^{2} \rightarrow 1+\frac{1}{\sqrt[p]{2^{p}+1}},}
\end{aligned}
$$

(3.12) must fail for some $0<\alpha_{1}<\widetilde{\alpha}_{1}<\alpha_{2}=\widetilde{\alpha}_{2} / 2<\widetilde{\alpha}_{2}$.

\section{Proposition 3.6 Suppose}

$$
\begin{aligned}
& \alpha_{1} \leq \cdots \leq \alpha_{k}<0=\alpha_{k+1}=\cdots=\alpha_{k+\ell}=0<\alpha_{k+\ell+1} \leq \cdots \alpha_{n}, \quad \text { and } \\
& \widetilde{\alpha}_{1} \leq \cdots \leq \widetilde{\alpha}_{k}<0=\widetilde{\alpha}_{k+1}=\cdots=\widetilde{\alpha}_{k+\ell}=0<\widetilde{\alpha}_{k+\ell+1} \leq \cdots \widetilde{\alpha}_{n} .
\end{aligned}
$$

Then given a permutation $\pi$ of $\{1,2, \cdots, n\}$, there exists another permutation $\tau$ of $\{1,2, \cdots, n\}$ such that

$$
1 \leq \tau(j) \leq k \text { for } 1 \leq j \leq k \text {, and } \tau(j)=j \text { for } j=k+1, \cdots, k+\ell
$$

and

$$
\varrho_{p}\left(\alpha_{i}, \widetilde{\alpha}_{\pi(i)}\right) \geq \varrho_{p}\left(\alpha_{i}, \widetilde{\alpha}_{\tau(i)}\right) \quad \text { for } i=1,2, \cdots, n \text {. }
$$

Proof: With the help of Property 6 of Proposition 3.1, a two-step proof can be given as follows.

1. Find a permutation $\tau_{1}$ such that $1 \leq \tau_{1}(j) \leq k+\ell$ for $1 \leq j \leq k+\ell$, and

$$
\varrho_{p}\left(\alpha_{i}, \widetilde{\alpha}_{\pi(i)}\right) \geq \varrho_{p}\left(\alpha_{i}, \widetilde{\alpha}_{\tau_{1}(i)}\right) \text { for } i=1,2, \cdots, n
$$

2. Find a permutation $\tau$ such that $1 \leq \tau(j) \leq k$ for $1 \leq j \leq k$, and $\tau(j)=j$ for $j=k+1, \cdots, k+\ell$, and

$$
\varrho_{p}\left(\alpha_{i}, \widetilde{\alpha}_{\tau_{1}(i)}\right) \geq \varrho_{p}\left(\alpha_{i}, \widetilde{\alpha}_{\tau_{2}(i)}\right) \text { for } i=1,2, \cdots, n .
$$

The detail is left to the reader.

\subsection{Barlow-Demmel-Veselić Relative Distance}

We introduce another relative distance between $\alpha$ and $\widetilde{\alpha}$ :

$$
\chi(\alpha, \widetilde{\alpha}) \stackrel{\text { def }}{=} \frac{|\alpha-\tilde{\alpha}|}{\sqrt{|\alpha \widetilde{\alpha}|}} .
$$

We treat $1 / 0=\infty$ and again $0 / 0 \equiv 0$. It was first used by Barlow and Demmel [1, $1990]$ and Demmel and Veselić $[9,1992]$ to define relative gaps between the spectra of two matrices. We call it the Barlow-Demmel-Veselić Relative Distance between $\alpha$ and $\widetilde{\alpha}$

Proposition 3.7 Let $\alpha, \widetilde{\alpha} \in \mathbf{C}$. 
1. $\chi(\alpha, \widetilde{\alpha}) \geq 0$; and $\chi(\alpha, \widetilde{\alpha})=0$ if and only if $\alpha=\widetilde{\alpha}$.

2. $\chi(\alpha, \widetilde{\alpha})=\chi(\widetilde{\alpha}, \alpha)$.

3. $\chi(\xi \alpha, \xi \widetilde{\alpha})=\chi(\alpha, \widetilde{\alpha})$ for all $0 \neq \xi \in \mathbf{C}$.

4. $\chi(1 / \alpha, 1 / \widetilde{\alpha})=\chi(\alpha, \widetilde{\alpha})$ for $\alpha \neq 0$ and $\tilde{\alpha} \neq 0$.

5. $\chi(\alpha, 0)=\infty$ if $\alpha \neq 0$.

6. if $\alpha, \widetilde{\alpha} \in \mathbf{R}$ and $\alpha \widetilde{\alpha}<0$, then $\chi(\alpha, \widetilde{\alpha}) \geq 2$.

7. if $\alpha, \beta, \widetilde{\alpha}, \widetilde{\beta} \in \mathbf{R}$ and $\alpha \leq \beta \leq \widetilde{\beta} \leq \widetilde{\alpha}$ and $\alpha \widetilde{\alpha} \geq 0$, then

$$
\chi(\alpha, \widetilde{\alpha}) \geq \chi(\beta, \widetilde{\beta}) .
$$

Proof: Properties 1-5 are easy to verify. Property 6 follows from when $\alpha \tilde{\alpha}<0$,

$$
\chi(\alpha, \widetilde{\alpha})=\frac{|\alpha-\tilde{\alpha}|}{\sqrt{|\alpha \tilde{\alpha}|}}=\frac{|\alpha|+|\widetilde{\alpha}|}{\sqrt{|\alpha \tilde{\alpha}|}} \geq \frac{2 \sqrt{|\alpha||\widetilde{\alpha}|}}{\sqrt{|\alpha \widetilde{\alpha}|}}=2,
$$

by the Cauchy-Schwarz inequality. To prove Property 7 , we notice that function $\frac{1}{x}-x$ for $0 \leq x \leq 1$ is monotonically decreasing and $0 \leq \alpha / \widetilde{\alpha} \leq \beta / \widetilde{\beta} \leq 1$; thus

$$
\chi(\alpha, \widetilde{\alpha})=\frac{1}{\sqrt{\alpha / \widetilde{\alpha}}}-\sqrt{\alpha / \widetilde{\alpha}} \geq \frac{1}{\sqrt{\beta / \widetilde{\beta}}}-\sqrt{\beta / \widetilde{\beta}}=\chi(\beta, \widetilde{\beta}),
$$

as was to be shown.

Remark: In Property 7 of Proposition 3.7, assumption $\alpha \widetilde{\alpha} \geq 0$ is essential, since inequality (3.14) is clearly violated if $\alpha<0<\beta<\widetilde{\beta} \leq \widetilde{\alpha}$ and $\beta$ is sufficiently close to 0 .

The following proposition establishes the topological equivalence between the classical measurement (see (3.1)) and our new relative distance $\chi$.

Proposition 3.8 Let $\alpha, \tilde{\alpha} \in \mathbf{R}$. If $0 \leq \epsilon<1$, then

$$
\left|\frac{\tilde{\alpha}}{\alpha}-1\right| \leq \epsilon \Rightarrow \chi(\alpha, \widetilde{\alpha}) \leq \frac{\epsilon}{\sqrt{1-\epsilon}}
$$

if $0 \leq \epsilon<2$, then

$$
\chi(\alpha, \tilde{\alpha}) \leq \epsilon \Rightarrow \max \left\{\left|\frac{\tilde{\alpha}}{\alpha}-1\right|,\left|\frac{\alpha}{\widetilde{\alpha}}-1\right|\right\} \leq\left(\frac{\epsilon}{2}+\sqrt{1+\frac{\epsilon^{2}}{4}}\right) \epsilon .
$$

Asymptotically,

$$
\lim _{\widetilde{\alpha} \rightarrow \alpha} \frac{\chi(\alpha, \widetilde{\alpha})}{\left|\frac{\widetilde{\alpha}}{\alpha}-1\right|}=1
$$

thus (3.15) and (3.16) are at least asymptotically sharp. 
Proof: $\left|\frac{\widetilde{\alpha}}{\alpha}-1\right| \leq \epsilon$ implies $\widetilde{\alpha}=\alpha(1+\delta)$ for some $\delta \in \mathbf{R}$ with $|\delta| \leq \epsilon$. So

$$
\chi(\alpha, \tilde{\alpha})=\frac{|\delta \alpha|}{\sqrt{\alpha^{2}(1+\delta)}} \leq \frac{\epsilon}{\sqrt{1-\epsilon}},
$$

as required. To prove (3.16), we set either $\xi=\alpha / \widetilde{\alpha}$ or $\xi=\widetilde{\alpha} / \alpha . \epsilon<2 \Rightarrow \xi>0$ (see Property 6 of Proposition 3.7). $\chi(\alpha, \widetilde{\alpha}) \stackrel{\text { def }}{=} \eta \leq \epsilon$ gives

$$
\frac{|\xi-1|}{\sqrt{\xi}}=\eta \Rightarrow \xi^{2}-\left(2+\eta^{2}\right) \xi+1=0
$$

solving which yields

$$
\xi=\frac{2+\eta^{2} \pm \sqrt{\left(2+\eta^{2}\right)^{2}-4}}{2}=1+\left(\frac{\eta}{2} \pm \sqrt{1+\frac{\eta^{2}}{4}}\right) \eta .
$$

Hence

$$
|\xi-1| \leq\left(\frac{\eta}{2}+\sqrt{1+\frac{\eta^{2}}{4}}\right) \eta \leq\left(\frac{\epsilon}{2}+\sqrt{1+\frac{\epsilon^{2}}{4}}\right) \epsilon
$$

as was to be shown.

Proposition 3.9 Let $\widetilde{\beta}=\beta(1+\delta)$. Assume that $|\beta| \leq|\alpha|$ and $|\delta| \leq \epsilon<1$, then

$$
\frac{\chi(\alpha, \beta)}{\sqrt{1-\epsilon}}+\frac{\epsilon}{\sqrt{1-\epsilon}} \geq \chi(\alpha, \tilde{\beta}) \geq \frac{\chi(\alpha, \beta)}{\sqrt{1+\epsilon}}-\frac{\epsilon}{\sqrt{1+\epsilon}} .
$$

Proof: Since $|\beta|(1-\epsilon) \leq|\widetilde{\beta}| \leq|\beta|(1+\epsilon)$ and $|\beta / \alpha| \leq 1$,

$$
\begin{aligned}
& \chi(\alpha, \widetilde{\beta})=\frac{|\alpha-\widetilde{\beta}|}{\sqrt{|\alpha \widetilde{\beta}|}} \geq \frac{|\alpha-\beta|-|\delta \beta|}{\sqrt{|\alpha \widetilde{\beta}|}} \geq \frac{|\alpha-\beta|-\epsilon|\beta|}{\sqrt{|\alpha \beta|(1+\epsilon)}} \geq \frac{\chi(\alpha, \beta)}{\sqrt{1+\epsilon}}-\frac{\epsilon}{\sqrt{1+\epsilon}}, \\
& \chi(\alpha, \tilde{\beta}) \leq \frac{|\alpha-\beta|+|\delta \beta|}{\sqrt{|\alpha \tilde{\beta}|}} \leq \frac{|\alpha-\beta|+\epsilon|\beta|}{\sqrt{|\alpha \beta|(1-\epsilon)}} \leq \frac{\chi(\alpha, \beta)}{\sqrt{1-\epsilon}}+\frac{\epsilon}{\sqrt{1-\epsilon}},
\end{aligned}
$$

as required.

Remark. Proposition 3.9, in contrast to Proposition 3.3, only provides bounds on how $\chi$ varies when its argument smaller in magnitude is perturbed a little. When both arguments are perturbed, following the lines of the proof above, one obtains

$$
\frac{\chi(\alpha, \beta)}{1-\epsilon}+\frac{\epsilon}{1-\epsilon} \frac{|\alpha|+|\beta|}{\sqrt{|\alpha \beta|}} \geq \chi(\widetilde{\alpha}, \widetilde{\beta}) \geq \frac{\chi(\alpha, \beta)}{1+\epsilon}-\frac{\epsilon}{1+\epsilon} \frac{|\alpha|+|\beta|}{\sqrt{|\alpha \beta|}},
$$

where $\widetilde{\alpha}=\alpha\left(1+\delta_{1}\right)$ and $\widetilde{\beta}=\beta\left(1+\delta_{2}\right)$ with $\left|\delta_{i}\right| \leq \epsilon$. The ratio $\frac{|\alpha|+|\beta|}{\sqrt{|\alpha \beta|}}$ which could be arbitrarily large plays a crucial role here. It can shown that

$$
2 \leq \frac{|\alpha|+|\beta|}{\sqrt{|\alpha \beta|}} \leq 2+\chi(\alpha, \beta)
$$


Proposition 3.10 For $\alpha, \tilde{\alpha} \in \mathbf{C}$, we have

$$
[2-\chi(\alpha, \widetilde{\alpha})] \chi(\alpha, \widetilde{\alpha}) \leq \chi\left(\alpha^{2}, \widetilde{\alpha}^{2}\right) \leq[2+\chi(\alpha, \widetilde{\alpha})] \chi(\alpha, \widetilde{\alpha})
$$

if, moreover, $\alpha, \widetilde{\alpha} \geq 0$, then

$$
2 \chi(\alpha, \widetilde{\alpha}) \leq \chi\left(\alpha^{2}, \widetilde{\alpha}^{2}\right)
$$

and the equality holds if and only if $\alpha=\widetilde{\alpha}$.

Proof: No proof is necessary if $\alpha=\widetilde{\alpha}=0$. Assume that at least one of $\alpha$ and $\widetilde{\alpha}$ is not zero. Notice that

$$
\chi\left(\alpha^{2}, \widetilde{\alpha}^{2}\right)=\frac{|\alpha+\widetilde{\alpha}|}{\sqrt{|\alpha \tilde{\alpha}|}} \frac{|\alpha-\widetilde{\alpha}|}{\sqrt{|\alpha \tilde{\alpha}|}}=\frac{|\alpha+\widetilde{\alpha}|}{\sqrt{|\alpha \tilde{\alpha}|}} \chi(\alpha, \widetilde{\alpha}) .
$$

To prove (3.18), without loss of any generality, we may assume that $|\alpha| \geq|\widetilde{\alpha}|$; then

$$
\begin{aligned}
& \frac{|\alpha+\widetilde{\alpha}|}{\sqrt{|\alpha \tilde{\alpha}|}}=\frac{|\alpha-\tilde{\alpha}+2 \tilde{\alpha}|}{\sqrt{|\alpha \tilde{\alpha}|}} \leq \frac{|\alpha-\tilde{\alpha}|}{\sqrt{|\alpha \tilde{\alpha}|}}+\frac{|2 \widetilde{\alpha}|}{\sqrt{|\alpha \widetilde{\alpha}|}} \leq \chi(\alpha, \widetilde{\alpha})+2, \\
& \frac{|\alpha+\widetilde{\alpha}|}{\sqrt{|\alpha \tilde{\alpha}|}}=\frac{|2 \alpha-(\alpha-\widetilde{\alpha})|}{\sqrt{|\alpha \tilde{\alpha}|}} \geq \frac{|2 \alpha|}{\sqrt{|\alpha \widetilde{\alpha}|}}-\frac{|\alpha-\widetilde{\alpha}|}{\sqrt{|\alpha \widetilde{\alpha}|}} \geq 2-\chi(\alpha, \widetilde{\alpha}) .
\end{aligned}
$$

These confirm (3.18). Now if $\alpha, \tilde{\alpha} \geq 0$, then

$$
\frac{|\alpha+\widetilde{\alpha}|}{\sqrt{|\alpha \widetilde{\alpha}|}}=\frac{\alpha+\widetilde{\alpha}}{\sqrt{\alpha \widetilde{\alpha}}} \geq \frac{2 \sqrt{\alpha \widetilde{\alpha}}}{\sqrt{\alpha \widetilde{\alpha}}}=2,
$$

as was to be shown.

Remark. There is no universal constant $c>0$, independent of $\alpha$ and $\widetilde{\alpha}$, such that for all $\alpha, \widetilde{\alpha} \in \mathbf{C}, \chi\left(\alpha^{2}, \widetilde{\alpha}^{2}\right)$ is bounded by $c \times \chi(\alpha, \widetilde{\alpha})$, unlike (3.9) in Proposition 3.4.

Proposition 3.11 For $\alpha, \tilde{\alpha} \in \mathbf{C}$,

$$
\varrho_{p}(\alpha, \widetilde{\alpha}) \leq 2^{-1 / p} \chi(\alpha, \widetilde{\alpha}),
$$

and the equality holds if and only if $|\alpha|=|\widetilde{\alpha}|$. If $\varrho_{p}(\alpha, \widetilde{\alpha})<2^{-1 / p}$, then

$$
\chi(\alpha, \widetilde{\alpha}) \leq \frac{2^{1 / p} \varrho_{p}(\alpha, \widetilde{\alpha})}{\sqrt{1-2^{1 / p} \varrho_{p}(\alpha, \widetilde{\alpha})}} .
$$

Proof: By the Cauchy-Schwarz inequality, we have

$$
|\alpha|^{p}+|\widetilde{\alpha}|^{p} \geq 2 \sqrt{|\alpha|^{p}|\widetilde{\alpha}|^{p}}=2(\sqrt{|\alpha \widetilde{\alpha}|})^{p} \Rightarrow \sqrt[p]{|\alpha|^{p}+|\widetilde{\alpha}|^{p}} \geq 2^{1 / p} \sqrt{|\alpha \widetilde{\alpha}|},
$$

from which the first inequality follows. To prove the second one, we notice that

$$
\chi(\alpha, \widetilde{\alpha})=\frac{|\alpha-\widetilde{\alpha}|}{\sqrt[p]{|\alpha|^{p}+|\widetilde{\alpha}|^{p}}} \cdot \frac{\sqrt[p]{|\alpha|^{p}+|\widetilde{\alpha}|^{p}}}{\sqrt{|\alpha \widetilde{\alpha}|}}=\frac{\sqrt[p]{|\alpha|^{p}+|\widetilde{\alpha}|^{p}}}{\sqrt{|\alpha \widetilde{\alpha}|}} \varrho_{p}(\alpha, \tilde{\alpha}) .
$$


Without loss of any generality, we may assume that $|\alpha| \leq|\widetilde{\alpha}|$. Under the condition $\varrho_{p}(\alpha, \widetilde{\alpha})<2^{-1 / p}$, we have

$$
\begin{aligned}
& \frac{\sqrt{|\alpha \widetilde{\alpha}|}}{\sqrt[p]{|\alpha|^{p}+|\widetilde{\alpha}|^{p}}}=\sqrt{\frac{|\alpha|}{\sqrt[p]{|\alpha|^{p}+|\widetilde{\alpha}|^{p}}} \cdot \frac{|\widetilde{\alpha}|}{\sqrt[p]{|\alpha|^{p}+|\widetilde{\alpha}|^{p}}}}=\sqrt{\frac{|\alpha-\widetilde{\alpha}+\widetilde{\alpha}|}{\sqrt[p]{|\alpha|^{p}+|\widetilde{\alpha}|^{p}}} \cdot \frac{|\widetilde{\alpha}|}{\sqrt[p]{|\alpha|^{p}+|\widetilde{\alpha}|^{p}}}} \\
& \geq \sqrt{\left(\frac{|\tilde{\alpha}|}{\sqrt[p]{|\alpha|^{p}+|\tilde{\alpha}|^{p}}}-\frac{|\alpha-\tilde{\alpha}|}{\sqrt[p]{|\alpha|^{p}+|\widetilde{\alpha}|^{p}}}\right) \cdot \frac{|\tilde{\alpha}|}{\sqrt[p]{|\alpha|^{p}+|\tilde{\alpha}|^{p}}}} \\
& =\sqrt{\left(\frac{1}{\sqrt[p]{1+\xi^{p}}}-\varrho_{p}(\alpha, \tilde{\alpha})\right) \cdot \frac{1}{\sqrt[p]{1+\xi^{p}}}} \quad \text { (Here, } \xi=\frac{|\alpha|}{|\widetilde{\alpha}|} \leq 1 \text { ) } \\
& \geq \sqrt{\left[2^{-1 / p}-\varrho_{p}(\alpha, \widetilde{\alpha})\right] 2^{-1 / p}}=2^{-1 / p} \sqrt{1-2^{1 / p} \varrho_{p}(\alpha, \widetilde{\alpha})}
\end{aligned}
$$

from which the second inequality now follows.

Proposition 3.11 is useful in that any bound with $\chi$ yields a bound with $\varrho_{p}$, and any bound with $\varrho_{p}$ yields a bound with $\chi$ with additional assumptions.

Now we consider again the question: what is the best way to pair two sequences of real numbers ordered as in (3.11)? With the help of Property 7 in Proposition 3.7 we can prove in the same way as proving Proposition 3.5 that

Proposition 3.12 If all $\alpha_{i}$ 's and $\widetilde{\alpha}_{j}$ 's are nonnegative and ordered as in (3.11), then

$$
\max _{1 \leq i \leq n} \chi\left(\alpha_{i}, \tilde{\alpha}_{i}\right)=\min _{\tau} \max _{1 \leq i \leq n} \chi\left(\alpha_{i}, \widetilde{\alpha}_{\tau(i)}\right)
$$

where the minimization is taken over all permutations $\tau$ of $\{1,2, \cdots, n\}$.

Remark. Proposition 3.12 may fail if not all $\alpha_{i}$ 's and $\widetilde{\alpha}_{j}$ 's are of the same sign. A counterexample is as follows: $n=2$ and

$$
\alpha_{1}=-1<\alpha_{2}=1 \quad \text { and } \quad \widetilde{\alpha}_{1}=\frac{1}{4}<\widetilde{\alpha}_{2}=2 .
$$

Then

$$
\begin{aligned}
& \max \left\{\chi\left(\alpha_{1}, \tilde{\alpha}_{1}\right), \chi\left(\alpha_{2}, \widetilde{\alpha}_{2}\right)\right\}=\max \{5 / 2,1 / \sqrt{2}\}=5 / 2 \\
& >3 / \sqrt{2}=\max \{3 / \sqrt{2}, 3 / 2\}=\max \left\{\chi\left(\alpha_{1}, \widetilde{\alpha}_{2}\right), \chi\left(\alpha_{2}, \widetilde{\alpha}_{1}\right)\right\} .
\end{aligned}
$$

Lemma 3.1 Let $0<\alpha_{1} \leq \alpha_{2}$ and $0<\widetilde{\alpha}_{1} \leq \widetilde{\alpha}_{2}$. Then

$$
\left[\chi\left(\alpha_{1}, \widetilde{\alpha}_{1}\right)\right]^{2}+\left[\chi\left(\alpha_{2}, \widetilde{\alpha}_{2}\right)\right]^{2} \leq\left[\chi\left(\alpha_{1}, \widetilde{\alpha}_{2}\right)\right]^{2}+\left[\chi\left(\alpha_{2}, \widetilde{\alpha}_{1}\right)\right]^{2},
$$

or in other words,

$$
\frac{\left(\widetilde{\alpha}_{1}-\alpha_{1}\right)^{2}}{\widetilde{\alpha}_{1} \alpha_{1}}+\frac{\left(\widetilde{\alpha}_{2}-\alpha_{2}\right)^{2}}{\widetilde{\alpha}_{2} \alpha_{2}} \leq \frac{\left(\widetilde{\alpha}_{2}-\alpha_{1}\right)^{2}}{\widetilde{\alpha}_{2} \alpha_{1}}+\frac{\left(\widetilde{\alpha}_{1}-\alpha_{2}\right)^{2}}{\widetilde{\alpha}_{1} \alpha_{2}}
$$

and the equality holds if and only if either $\alpha_{1}=\alpha_{2}$ or $\widetilde{\alpha}_{1}=\widetilde{\alpha}_{2}$. 
Proof: It can be verified that

$$
\begin{array}{r}
\frac{\left(\widetilde{\alpha}_{1}-\alpha_{1}\right)^{2}}{\widetilde{\alpha}_{1} \alpha_{1}}+\frac{\left(\widetilde{\alpha}_{2}-\alpha_{2}\right)^{2}}{\widetilde{\alpha}_{2} \alpha_{2}}-\frac{\left(\widetilde{\alpha}_{2}-\alpha_{1}\right)^{2}}{\widetilde{\alpha}_{2} \alpha_{1}}-\frac{\left(\widetilde{\alpha}_{1}-\alpha_{2}\right)^{2}}{\widetilde{\alpha}_{1} \alpha_{2}} \\
=-\frac{\left(\alpha_{2}-\alpha_{1}\right)\left(\widetilde{\alpha}_{2}-\widetilde{\alpha}_{1}\right)\left(\widetilde{\alpha}_{1} \widetilde{\alpha}_{2}+\alpha_{1} \alpha_{2}\right)}{\widetilde{\alpha}_{1} \alpha_{1} \widetilde{\alpha}_{2} \alpha_{2}} \leq 0,
\end{array}
$$

and the equality holds if and only if either $\alpha_{1}=\alpha_{2}$ or $\widetilde{\alpha}_{1}=\widetilde{\alpha}_{2}$.

Armed with Lemma 3.1, by similar reasoning as in the proof of Proposition 3.5, one can show that

Proposition 3.13 Let $\left\{\alpha_{1}, \cdots, \alpha_{n}\right\}$ and $\left\{\widetilde{\alpha}_{1}, \cdots, \widetilde{\alpha}_{n}\right\}$ be two sequences of $n$ positive numbers ordered ascendingly as in (3.11). Then

$$
\sum_{i=1}^{n}\left[\chi\left(\alpha_{i}, \tilde{\alpha}_{i}\right)\right]^{2}=\min _{\tau} \sum_{i=1}^{n}\left[\chi\left(\alpha_{i}, \tilde{\alpha}_{\tau(i)}\right)\right]^{2},
$$

where the minimization is taken over all permutations $\tau$ of $\{1,2, \cdots, n\}$.

Remark. Proposition 3.13 may fail if not all $\alpha_{i}$ 's and $\widetilde{\alpha}_{j}$ 's are of the same sign. Here is a counterexample: $n=2$ and

$$
\alpha_{1}=-2<\alpha_{2}=1 \quad \text { and } \quad \widetilde{\alpha}_{1}=1<\widetilde{\alpha}_{2}=2 .
$$

Then

$$
\begin{aligned}
& {\left[\chi\left(\alpha_{1}, \widetilde{\alpha}_{1}\right)\right]^{2}+\left[\chi\left(\alpha_{2}, \widetilde{\alpha}_{2}\right)\right]^{2}=(3 / \sqrt{2})^{2}+(1 / \sqrt{2})^{2}=5} \\
& >4=(4 / \sqrt{4})^{2}+0^{2}=\left[\chi\left(\alpha_{1}, \widetilde{\alpha}_{2}\right)\right]^{2}+\left[\chi\left(\alpha_{2}, \widetilde{\alpha}_{1}\right)\right]^{2}
\end{aligned}
$$

\subsection{Are $\varrho_{p}$ and $\chi$ Metrics?}

Let $\mathbf{X}$ be a space. Recall that a function $d: \mathrm{X} \times \mathbf{X} \mapsto[0, \infty)$ is called a metric if it has the following three properties: for $\alpha, \beta, \gamma \in \mathrm{X}$

1. $d(\alpha, \beta)=0$ if and only if $\alpha=\beta$;

2. $d(\alpha, \beta)=d(\beta, \alpha)$;

3. $d(\alpha, \gamma) \leq d(\alpha, \beta)+d(\beta, \gamma)$.

This definition excludes immediately the possibility that $\chi$ is a metric on $\mathbf{C}$, nor even on $\mathbf{R}$ since $\chi(\alpha, 0)=\infty$ for $\alpha \neq 0$. To get around this, we call $d: \mathbf{X} \times \mathbf{X} \mapsto[0, \infty]$ a generalized metric if it possesses the above three properties.

From Propositions 3.1 and 3.7, we see that functions $\varrho_{p}$ and $\chi$ on $\mathbf{C} \times \mathbf{C}$ satisfy the first two properties in the definition of a (generalized) metric. Naturally, we would like to 
ask: Is $\varrho_{p}$ a metric on $\mathbf{C}$ ? is $\chi$ a generalized metric on $\mathbf{C}$ ? In other words, we like to know whether for $\alpha, \beta, \gamma \in \mathbf{C}$

$$
\begin{aligned}
\varrho_{p}(\alpha, \gamma) & \leq \varrho_{p}(\alpha, \beta)+\varrho_{p}(\beta, \gamma) ? \\
\chi(\alpha, \gamma) & \leq \chi(\alpha, \beta)+\chi(\beta, \gamma) ?
\end{aligned}
$$

A quick answer to (3.21) is $N o$, even for $\alpha, \beta, \gamma>0$, by the following proposition.

Proposition 3.14 For $0 \leq \alpha \leq \beta \leq \gamma$, we have

$$
\chi(\alpha, \gamma) \geq \chi(\alpha, \beta)+\chi(\beta, \gamma)
$$

The equality holds if and only if either $\alpha=\beta$ or $\beta=\gamma$.

Proof: It can be verified that

$$
\chi(\alpha, \gamma)-\chi(\alpha, \beta)-\chi(\beta, \gamma)=\frac{(\sqrt{\gamma}-\sqrt{\alpha})(\sqrt{\gamma}-\sqrt{\beta})(\sqrt{\beta}-\sqrt{\alpha})}{\sqrt{\alpha \beta \gamma}} \geq 0 .
$$

It is zero if and only if $\alpha=\beta$ or $\beta=\gamma .(\alpha=\gamma$ implies $\alpha=\beta$ and $\beta=\gamma$.

Inequality (3.22) is exactly the opposite of (3.21) which, otherwise, would be true if $\chi$ were a metric on $\mathbf{R}$.

However, it takes a few pages of work to answer (3.20) for $\alpha, \beta, \gamma \in \mathbf{R}$. We leave the detail to Appendix A, where it is proved:

Proposition 3.15 (3.20) holds for $\alpha, \beta, \gamma \in \mathbf{R}$, and thus $\varrho_{p}$ for $1 \leq p \leq \infty$ is a metric on $\mathbf{R}$.

Still the question whether $\varrho_{p}$ is a metric on $\mathbf{C}$ is open.

\section{Known Perturbation Theorems for Eigenvalue and Sin- gular Value Variations}

In this section, we will briefly review several most celebrated theorems for eigenvalue and singular value variations which will be extended later. Most of these theorems can be found in Bhatia [3, 1987], Golub and Van Loan [14, 1989], Parlett [33, 1980] and Stewart and Sun [35, 1990]. Notation introduced at the beginning of $\$ 2$ will be followed strictly.

Hoffman and Wielandt $[16,1953]$ proved

Theorem 4.1 (Hoffman-Wielandt) If $A$ and $\tilde{A}$ are normal, then there is a permutation $\tau$ of $\{1,2, \cdots, n\}$ such that

$$
\sqrt{\sum_{i=1}^{n}\left|\lambda_{i}-\tilde{\lambda}_{\tau(i)}\right|^{2}} \leq\|\tilde{A}-A\|_{\mathrm{F}} .
$$


For a nonsingular matrix $Y \in \mathbf{C}^{n \times n}$, the (spectral) condition number $\kappa(Y)$ is defined as

$$
\kappa(Y) \stackrel{\text { def }}{=}\|Y\|_{2}\left\|Y^{-1}\right\|_{2} \text {. }
$$

Theorem 4.1 was generalized by Sun $[38,1984]$ and Zhang $[43,1986]$ to two diagonalizable matrices.

Theorem 4.2 (Sun-Zhang) Assume that both $A$ and $\tilde{A}$ are diagonalizable and admit the following decompositions

$$
A=X \Lambda X^{-1} \quad \text { and } \quad \tilde{A}=\tilde{X} \tilde{\Lambda} \tilde{X}^{-1}
$$

where $X$ and $\tilde{X}$ are nonsingular and

$$
\Lambda=\operatorname{diag}\left(\lambda_{1}, \lambda_{2}, \cdots, \lambda_{n}\right) \quad \text { and } \quad \tilde{\Lambda}=\operatorname{diag}\left(\tilde{\lambda}_{1}, \tilde{\lambda}_{2}, \cdots, \tilde{\lambda}_{n}\right)
$$

Then there is a permutation $\tau$ of $\{1,2, \cdots, n\}$ such that

$$
\sqrt{\sum_{i=1}^{n}\left|\lambda_{i}-\tilde{\lambda}_{\tau(i)}\right|^{2}} \leq \kappa(X) \kappa(\tilde{X})\|\tilde{A}-A\|_{\mathrm{F}} .
$$

Such matrices $A$ and $\tilde{A}$ as described in Theorem 4.2 are called normalizable. Sun [38, 1984 ] proved this theorem when $A$ is normal and $\tilde{A}$ normalizable; later Zhang [43, 1986] found that a slight modification of Sun's proof serves the case when both $A$ and $\tilde{A}$ are normalizable.

We will consider unitarily invariant norms $\|\cdot\| \|$ of matrices. In this we follow Mirsky [31, $1960]$ and Stewart and Sun $[35,1990]$. That a norm $\|\cdot\|$ is unitarily invariant on $\mathbf{C}^{m \times n}$ means that it satisfies, besides the usual properties of any norm, also

1. $\|U Y V\|=\|Y\|$, for any $U \in \mathbf{U}_{m}$, and $V \in \mathbf{U}_{n}$;

2. $\|Y\|=\|Y\|_{2}$, for any $Y \in \mathbf{C}^{m \times n}$ with $\operatorname{rank}(Y)=1$.

Two unitarily invariant norms used frequently are the spectral norm $\|\cdot\|_{2}$ and the Frobenius norm $\|\cdot\|_{F}$. Let $\|\cdot\|$ be a unitarily invariant norm on some matrix space. The following inequalities $[35, \mathrm{p} .80]$ will be employed frequently in the rest of this paper:

$$
\|W Y\| \leq\|W\|_{2}\|Y\| \text { and }\|Y Z\| \leq\|Y\|\|Z\|_{2} .
$$

Theorem 4.3 Suppose that $A$ and $\tilde{A}$ are both Hermitian, and that their eigenvalues are ordered descendingly as in (2.2). Then for any unitarily invariant norm $\|\cdot\|$

$$
\left\|\operatorname{diag}\left(\lambda_{1}-\tilde{\lambda}_{1}, \lambda_{2}-\tilde{\lambda}_{2}, \cdots, \lambda_{n}-\tilde{\lambda}_{n}\right)\right\| \leq\|A-\tilde{A}\| .
$$

Theorem 4.3 was proved by Weyl $[40,1912]$ for the spectral norm and by Loewner [27, 1934] for the Frobenius norm. Also, for the Frobenius norm it is a corollary of Theorem 4.1 by Hoffman and Wielandt [16, 1953]. For all unitarily invariant norms, (4.3) was proved by Mirsky [31, 1960]. He derived it from a theorem of Lidskii $[26,1950]$ and Wielandt [41, 1955].

Extensions to Theorem 4.3 have been made in the literature. The following theorem is due to $\operatorname{Li}[25,1996]$ and $\operatorname{Lu}[28,1994]$. 
Theorem 4.4 To the hypotheses of Theorem 4.2 adds this: all $\lambda_{i}$ 's and $\tilde{\lambda}_{j}$ 's are real and are ordered descendingly as in (2.2). Then for any unitarily invariant norm $\|\cdot\|$

$$
\left\|\operatorname{diag}\left(\lambda_{1}-\tilde{\lambda}_{1}, \lambda_{2}-\tilde{\lambda}_{2}, \cdots, \lambda_{n}-\tilde{\lambda}_{n}\right)\right\| \leq \sqrt{\kappa(X) \kappa(\tilde{X})}\|A-\tilde{A}\| .
$$

Such matrices $A$ and $\widetilde{A}$ as described in Theorem 4.4 are called symmetrizable. Inequality (4.4) for $\|\cdot\|=\|\cdot\|_{2}$ was proved by Lu [28, 1994]; for all unitarily invariant norms it is due to Li $[25,1996]$. This inequality improves substantially

$$
\left\|\operatorname{diag}\left(\lambda_{1}-\tilde{\lambda}_{1}, \lambda_{2}-\tilde{\lambda}_{2}, \cdots, \lambda_{n}-\tilde{\lambda}_{n}\right)\right\| \leq \kappa(X) \kappa(\tilde{X})\|A-\tilde{A}\| \|
$$

due to Bhatia, Davis and Kittaneh [4, 1991]. A brief history behind inequality (4.5) is as follows: It was proved by Kahan $[20,1975]$ for the spectral norm, and for the Frobenius norm it can be deduced without much difficulty from a theorem in Kahan [19, 1967]; also for Frobenius norm it is a corollary of Theorem 4.2 by Sun $[38,1984]$ and Zhang [43, 1986]. For all unitarily invariant norms, it is due to Bhatia, Davis and Kittaneh [4, 1991]. For other improvements of inequality (4.5), the reader is referred to $\operatorname{Li}[25,1996]$.

Inequality (4.3) for the spectral norm was generalized also to $\ell_{p}$ operator norm. The $p$-Hölder norm of a vector $y=\left(\gamma_{i}\right) \in \mathbf{C}^{n}$ is defined by

$$
\|y\|_{p} \stackrel{\text { def }}{=} \sqrt[p]{\sum_{i=1}^{n}\left|\gamma_{i}\right|^{p}}
$$

The $\ell_{p}$-operator norm of a matrix $Y \in \mathbf{C}^{m \times n}$ is defined by

$$
\|Y\|_{p} \stackrel{\text { def }}{=} \max _{\|y\|_{p}=1}\|Y y\|_{p}
$$

If $Y$ is square and nonsingular, its $\ell_{p}$ condition number is defined by

$$
\kappa_{p}(Y) \stackrel{\text { def }}{=}\|Y\|_{p}\left\|Y^{-1}\right\|_{p} .
$$

Clearly, $\kappa_{2}(\cdot)=\kappa(\cdot)$, the (spectral) condition number. The following theorem is due to Li $[23$, p.225, 1993].

Theorem $4.5(\mathrm{Li})$ Under the conditions of Theorem 4.4. Then

$$
\max _{1 \leq i \leq n}\left|\lambda_{i}-\tilde{\lambda}_{i}\right| \leq \kappa_{p}(X) \kappa_{p}(\tilde{X})\|A-\tilde{A}\|_{p}
$$

where $1 \leq p \leq \infty$.

Remark. It would be interesting to know whether $\kappa_{p}(X) \kappa_{p}(\tilde{X})$ in inequality (4.6) could be improved to $\sqrt{\kappa_{p}(X) \kappa_{p}(\tilde{X})}$ as a similar thing happened between (4.4) and (4.5).

Generally, if one of $A$ and $\tilde{A}$ is diagonalizable and the other is arbitrary, we have the following result due to Bauer and Fike ${ }^{3}[2,1960]$.

\footnotetext{
${ }^{3}$ One can prove a slightly more stronger inequality than (4.7)

$$
|\tilde{\lambda}-\lambda| \leq\left\|X^{-1}(\widetilde{A}-A) X\right\|_{2} .
$$
}


Theorem 4.6 (Bauer-Fike) Assume $A$ is diagonalizable, i.e.,

$$
A=X \Lambda X^{-1}, \quad \text { where } \Lambda=\operatorname{diag}\left(\lambda_{1}, \cdots, \lambda_{n}\right) .
$$

Then for any $\tilde{\lambda} \in \lambda(\tilde{A})$, there exists a $\lambda \in \lambda(A)$ such that

$$
|\widetilde{\lambda}-\lambda| \leq \kappa(X)\|\tilde{A}-A\|_{2} \text {. }
$$

Regarding singular value perturbations, the following theorem was established in Mirsky [31, 1960], based on results from Lidskii [26, 1950] and Wielandt [41, 1955].

Theorem 4.7 For any unitarily invariant norm $\|\cdot\|$, we have

$$
\left\|\operatorname{diag}\left(\sigma_{1}-\tilde{\sigma}_{1}, \sigma_{2}-\tilde{\sigma}_{2}, \cdots, \sigma_{n}-\tilde{\sigma}_{n}\right)\right\| \leq\|B-\tilde{B}\| .
$$

\section{Relative Perturbation Theorems for Nonnegative Defi- nite Matrix Eigenvalues and for Singular Values}

This section is devoted to the relative perturbation theory for eigenvalues of nonnegative definite matrices and for singular values. The following problems will be considered.

- Eigenvalue problems:

1. $A$ and $\tilde{A}=D^{*} A D$, where $A$ is nonnegative definite, and $D$ is close to some unitary matrix.

2. $A=S^{*} H S$ and $\widetilde{A}=S^{*} \tilde{H} S$, where $H$ is positive definite and $\left\|H^{-1}\right\|_{2}\|\tilde{H}-H\|_{2}<$ 1 , and $S$ is some square matrix.

- Singular value problems:

1. $B$ and $\widetilde{B}=D_{1}^{*} B D_{2}$, where $D_{1}$ and $D_{2}$ are close to some unitary matrices of suitable dimensions.

2. $B=G S$ and $\widetilde{B}=\widetilde{G} S$, where $G$ is nonsingular and $\left\|G^{-1}\right\|_{2}\|\widetilde{G}-G\|_{2}<1$, and $S$ is some square matrix.

Theorems presented here are often sharper than these in the next section when applying to nonnegative definite matrices. We will make this more concrete in the coming section.

\subsection{Eigenvalue Variations for $A$ and $\tilde{A}=D^{*} A D$}

Theorem 5.1 Let $A$ and $\tilde{A}=D^{*} A D$ be two $n \times n$ Hermitian matrices with eigenvalues (2.1) ordered descendingly as in (2.2), where $D$ is nonsingular. Assume that $A$ is nonnegative definite ${ }^{4}$. Then

$$
\begin{aligned}
\max _{1 \leq i \leq n} \chi\left(\lambda_{i}, \tilde{\lambda}_{i}\right) & \leq\left\|D^{*}-D^{-1}\right\|_{2}, \\
\sqrt{\sum_{i=1}^{n}\left[\chi\left(\lambda_{i}, \tilde{\lambda}_{i}\right)\right]^{2}} & \leq\left\|D^{*}-D^{-1}\right\|_{\mathrm{F}} .
\end{aligned}
$$

\footnotetext{
${ }^{4}$ Then $\widetilde{A}$ must be nonnegative definite as well.
} 
It is easy to relate the right-hand sides of the inequalities (5.1) and (5.2) to the singular values of $D$. In fact, let the singular value decomposition (SVD) of $D$ be

$$
D=U_{\mathrm{d}} \Sigma_{\mathrm{d}} V_{\mathrm{d}}^{*}
$$

where $U_{\mathrm{d}}$ and $V_{\mathrm{d}}$ are unitary, and $\Sigma_{\mathrm{d}}$ is a diagonal matrix whose diagonal entries are the singular values of $D$. One has for any unitarily invariant norm $\|\cdot\|$

$$
\left\|D^{*}-D^{-1}\right\|\|=\| V_{\mathrm{d}}\left(\Sigma_{\mathrm{d}}-\Sigma_{\mathrm{d}}^{-1}\right) U_{\mathrm{d}}^{*}\|\|=\left\|\Sigma_{\mathbf{d}}-\Sigma_{\mathrm{d}}^{-1}\right\| \|
$$

\subsection{Singular Value Variations for $B$ and $\widetilde{B}=D_{1}^{*} B D_{2}$}

Theorem 5.2 Let $B$ and $\widetilde{B}=D_{1}^{*} B D_{2}$ be two $m \times n$ matrices with singular values (2.3) ordered descendingly as in (2.4), where $D_{1}$ and $D_{2}$ are square and nonsingular. If $\| D_{1}^{*}-$ $D_{1}^{-1}\left\|_{2}\right\| D_{2}^{*}-D_{2}^{-1} \|_{2}<32$, then

$$
\begin{aligned}
\max _{1 \leq i \leq n} \chi\left(\sigma_{i}, \tilde{\sigma}_{i}\right) & \leq \frac{1}{2} \cdot \frac{\left\|D_{1}^{*}-D_{1}^{-1}\right\|_{2}+\left\|D_{2}^{*}-D_{2}^{-1}\right\|_{2}}{1-\frac{1}{32}\left\|D_{1}^{*}-D_{1}^{-1}\right\|_{2}\left\|D_{2}^{*}-D_{2}^{-1}\right\|_{2}}, \\
\sqrt{\sum_{i=1}^{n}\left[\chi\left(\sigma_{i}, \tilde{\sigma}_{i}\right)\right]^{2}} & \leq \frac{1}{2} \cdot \frac{\left\|D_{1}^{*}-D_{1}^{-1}\right\|_{\mathrm{F}}+\left\|D_{2}^{*}-D_{2}^{-1}\right\|_{\mathrm{F}}}{1-\frac{1}{32}\left\|D_{1}^{*}-D_{1}^{-1}\right\|_{2}\left\|D_{2}^{*}-D_{2}^{-1}\right\|_{2}} .
\end{aligned}
$$

Now, Let's mention a possible application of Theorem 5.2. It has something to do with deflation in computing the singular value decomposition of a bidiagonal matrix. For more details, the reader is referred to $[6,8,10,30]$. We formulate the application into a corollary.

Corollary 5.1 Assume in Theorem 5.2, one of $D_{1}$ and $D_{2}$ is the identity matrix and the other takes the form

$$
D=\left(\begin{array}{cc}
I & X \\
& I
\end{array}\right),
$$

where $X$ is a matrix of suitable dimensions. With the notation of Theorem 5.2, we have

$$
\begin{aligned}
\max _{1 \leq i \leq n} \chi\left(\sigma_{i}, \tilde{\sigma}_{i}\right) & \leq \frac{1}{2}\|X\|_{2}, \\
\sqrt{\sum_{i=1}^{n}\left[\chi\left(\sigma_{i}, \tilde{\sigma}_{i}\right)\right]^{2}} & \leq \frac{1}{\sqrt{2}}\|X\|_{\mathrm{F}} .
\end{aligned}
$$

Proof: Notice that

$$
D^{*}-D^{-1}=\left(\begin{array}{cc}
I & \\
X^{*} & I
\end{array}\right)-\left(\begin{array}{cc}
I & -X \\
& I
\end{array}\right)=\left(\begin{array}{cc} 
& X \\
X^{*} &
\end{array}\right),
$$

and thus $\left\|D^{*}-D^{-1}\right\|_{2}=\|X\|_{2}$ and $\left\|D^{*}-D^{-1}\right\|_{\mathrm{F}}=\sqrt{2}\|X\|_{\mathrm{F}}$. 
It was proved by Eisenstat and Ipsen $[10,1993]$ that

$$
\left|\widetilde{\sigma}_{i}-\sigma_{i}\right| \leq\|X\|_{2} \sigma_{i}, \quad \text { or equivalently } \quad\left|\frac{\widetilde{\sigma}_{i}}{\sigma_{i}}-1\right| \leq\|X\|_{2} \text {. }
$$

Our inequality (5.6) is sharper by roughly a factor $1 / 2$, as long as $\|X\|_{2}$ is small. As a matter of fact, it follows from (5.6) and Proposition 3.8 that if $\|X\|_{2}<4$ then

$$
\left|\frac{\widetilde{\sigma}_{i}}{\sigma_{i}}-1\right| \leq\left(\frac{\|X\|_{2}}{4}+\sqrt{1+\frac{\|X\|_{2}^{2}}{16}}\right) \frac{\|X\|_{2}}{2}=\frac{\|X\|_{2}}{2}+O\left(\left(\frac{\|X\|_{2}}{4}\right)^{2}\right) .
$$

Our inequality (5.7) is the first of its kind.

Theorem 5.3 Let $B$ and $\widetilde{B}=D_{1}^{*} B D_{2}$ be two $m \times n$ matrices with singular values (2.3) ordered descendingly as in (2.4), where $D_{1}$ and $D_{2}$ are square and nonsingular. Then

$$
\begin{aligned}
\max _{1 \leq i \leq n} \varrho_{p}\left(\sigma_{i}, \tilde{\sigma}_{i}\right) & \leq \frac{1}{2^{1+1 / p}}\left(\left\|D_{1}^{*}-D_{1}^{-1}\right\|_{2}+\left\|D_{2}^{*}-D_{2}^{-1}\right\|_{2}\right), \\
\sqrt{\sum_{i=1}^{n}\left[\varrho_{p}\left(\sigma_{i}, \tilde{\sigma}_{i}\right)\right]^{2}} & \leq \frac{1}{2^{1+1 / p}}\left(\left\|D_{1}^{*}-D_{1}^{-1}\right\|_{\mathrm{F}}+\left\|D_{2}^{*}-D_{2}^{-1}\right\|_{\mathrm{F}}\right) .
\end{aligned}
$$

A straightforward combination of Proposition 3.11 and Theorem 5.2 will lead to bounds that are weaker than these in Theorem 5.3 by a factor $\left(1-\frac{1}{32}\left\|D_{1}^{*}-D_{1}^{-1}\right\|_{2}\left\|D_{2}^{*}-D_{2}^{-1}\right\|_{2}\right)^{-1}$ which may play an insubstantial role because $\left\|D_{1}^{*}-D_{1}^{-1}\right\|_{2}|| D_{2}^{*}-D_{2}^{-1} \|_{2}$ is of second order.

\subsection{Graded Matrices}

Theorem 5.4 Let $A=S^{*} H S$ and $\tilde{A}=S^{*} \tilde{H} S$ be two $n \times n$ nonnegative definite Hermitian matrices with eigenvalues (2.1) ordered descendingly as in (2.2), and let $\Delta H=\widetilde{H}-H$. If $\left\|H^{-1}\right\|_{2}\|\Delta H\|_{2}<1$, then

$$
\begin{aligned}
\max _{1 \leq i \leq n} \chi\left(\lambda_{i}, \tilde{\lambda}_{i}\right) & \leq\left\|\left(I+H^{-1 / 2}(\Delta H) H^{-1 / 2}\right)^{1 / 2}-\left(I+H^{-1 / 2}(\Delta H) H^{-1 / 2}\right)^{-1 / 2}\right\|_{2} \\
& \leq \frac{\left\|H^{-1}\right\|_{2}\|\Delta H\|_{2}}{\sqrt{1-\left\|H^{-1}\right\|_{2}\|\Delta H\|_{2}}} \\
\sqrt{\sum_{i=1}^{n}\left[\chi\left(\lambda_{i}, \tilde{\lambda}_{i}\right)\right]^{2}} & \leq\left\|\left(I+H^{-1 / 2}(\Delta H) H^{-1 / 2}\right)^{1 / 2}-\left(I+H^{-1 / 2}(\Delta H) H^{-1 / 2}\right)^{-1 / 2}\right\|_{\mathrm{F}} \\
& \leq \frac{\left\|H^{-1}\right\|_{2}\|\Delta H\|_{\mathrm{F}}}{\sqrt{1-\left\|H^{-1}\right\|_{2}\|\Delta H\|_{2}}} .
\end{aligned}
$$

The last inequality in (5.11) is derivable from a bound due to Demmel and Veselić $[9$, 1992] (see (7.10) below). 
Theorem 5.5 Let $B=G S$ and $\widetilde{B}=\widetilde{G} S$ be two $n \times n$ matrices with singular values (2.3) ordered descendingly as in (2.4), where $G$ and $\widetilde{G}$ are nonsingular, and let $\Delta G=\widetilde{G}-G$. If $\|\Delta G\|_{2}\left\|G^{-1}\right\|_{2}<1$, then

$$
\begin{aligned}
\max _{1 \leq i \leq n} \chi\left(\sigma_{i}, \tilde{\sigma}_{i}\right) & \leq \frac{1}{2}\left\|\left(I+(\Delta G) G^{-1}\right)^{*}-\left(I+(\Delta G) G^{-1}\right)^{-1}\right\|_{2} \\
& \leq\left(\frac{\left\|(\Delta G) G^{-1}+G^{-*}(\Delta G)^{*}\right\|_{2}}{\left\|(\Delta G) G^{-1}\right\|_{2}}+\frac{\left\|(\Delta G) G^{-1}\right\|_{2}}{1-\left\|(\Delta G) G^{-1}\right\|_{2}}\right) \frac{\left\|(\Delta G) G^{-1}\right\|_{2}}{2} \\
& \leq\left(1+\frac{1}{1-\left\|G^{-1}\right\|_{2}\|\Delta G\|_{2}}\right) \frac{\left\|G^{-1}\right\|_{2}\|\Delta G\|_{2}}{2} \\
\sqrt{\sum_{i=1}^{n}\left[\chi\left(\sigma_{i}, \tilde{\sigma}_{i}\right)\right]^{2}} & \leq \frac{1}{2}\left\|\left(I+(\Delta G) G^{-1}\right)^{*}-\left(I+(\Delta G) G^{-1}\right)^{-1}\right\|_{\mathrm{F}} \\
& \leq\left(\frac{\left\|(\Delta G) G^{-1}+G^{-*}(\Delta G)^{*}\right\|_{\mathrm{F}}}{\left\|(\Delta G) G^{-1}\right\|_{\mathrm{F}}} \frac{\left\|(\Delta G) G^{-1}\right\|_{2}}{1-\left\|(\Delta G) G^{-1}\right\|_{2}}\right) \frac{\left\|(\Delta G) G^{-1}\right\|_{\mathrm{F}}}{2} \\
& \leq\left(1+\frac{1}{1-\left\|G^{-1}\right\|_{2}\|\Delta G\|_{2}}\right) \frac{\left\|G^{-1}\right\|_{2}\|\Delta G\|_{\mathrm{F}}}{2} .
\end{aligned}
$$

The last inequality in (5.13) is derivable from a bound due to Mathias [29, 1994] (see (7.12) below).

Remark. It is interesting to notice that if $(\Delta G) G^{-1}$ is very skew, then $\chi\left(\sigma_{i}, \tilde{\sigma}_{i}\right)=$ $o\left(\left\|(\Delta G) G^{-1}\right\|_{2}\right)$, especially

$$
\left\|(\Delta G) G^{-1}+G^{-*}(\Delta G)^{*}\right\|_{2}=O\left(\left\|(\Delta G) G^{-1}\right\|_{2}^{2}\right) \Rightarrow \chi\left(\sigma_{i}, \widetilde{\sigma}_{i}\right)=O\left(\left\|(\Delta G) G^{-1}\right\|_{2}^{2}\right) .
$$

\section{Relative Perturbation Theorems for Non-Definite Ma- trix Eigenvalues}

This section is devoted to the perturbation theory with $\varrho_{p}$ for the following matrix eigenvalue problems.

1. $A$ and $\tilde{A}=D^{*} A D$ for the Hermitian case, where $D$ is nonsingular and close to $I$ or more generally to a unitary matrix.

2. $A$ and $\widetilde{A}=D_{1}^{*} A D_{2}$ for a general diagonalizable case, where $D_{1}$ and $D_{2}$ are nonsingular and close to $I$ or more generally to some unitary matrix.

Comparisons among theorems in this section and these in the previous section will be conducted. The following theorem is a generalization of Theorems 4.1 and 4.2.

Theorem 6.1 Assume that $n \times n$ matrix $A$ is perturbed to $\tilde{A}=D_{1}^{*} A D_{2}$ and both $D_{1}$ and $\mathrm{D}_{2}$ are nonsingular. Assume also that both $A$ and $\tilde{A}$ are diagonalizable and admit 
the decompositions as described in (4.1) and (4.2). Then there is a permutation $\tau$ of $\{1,2, \cdots, n\}$ such that

$$
\begin{gathered}
\sqrt{\sum_{i=1}^{n}\left[\varrho_{2}\left(\lambda_{i}, \tilde{\lambda}_{\tau(i)}\right)\right]^{2}} \leq \min \left\{\left\|\tilde{X}^{-1}\right\|_{2}\|X\|_{2} \sqrt{\left\|X^{-1}\left(I-D_{2}\right) \tilde{X}\right\|_{\mathrm{F}}^{2}+\left\|X^{-1}\left(D_{1}^{-*}-I\right) \tilde{X}\right\|_{\mathrm{F}}^{2}}\right. \\
\left.\left\|X^{-1}\right\|_{2}\|\tilde{X}\|_{2} \sqrt{\left\|\tilde{X}-1\left(I-D_{1}^{*}\right) X\right\|_{\mathrm{F}}^{2}+\left\|\tilde{X}^{-1}\left(D_{2}^{-1}-I\right) X\right\|_{\mathrm{F}}^{2}}\right\} \\
\leq \kappa(X) \kappa(\tilde{X}) \min \left\{\sqrt{\left\|I-D_{1}\right\|_{\mathrm{F}}^{2}+\left\|I-D_{2}^{-1}\right\|_{\mathrm{F}}^{2}}, \sqrt{\left\|I-D_{1}^{-1}\right\|_{\mathrm{F}}^{2}+\left\|I-D_{2}\right\|_{\mathrm{F}}^{2}}\right\} .
\end{gathered}
$$

For any given $U \in \mathbf{U}_{n}, U \tilde{A} U^{*}=\left(D_{1} U^{*}\right)^{*} A D_{2} U^{*}$ has the same eigenvalues as $\widetilde{A}$ does, and moreover from (4.1) $U \widetilde{A} U^{*}=\left(\tilde{X} U^{*}\right)^{-1} \widetilde{\Lambda} \tilde{X} U^{*}$. Applying Theorem 6.1 to matrices $A$ and $U \tilde{A} U^{*}$ leads to the following theorem which we will refer as Theorem $6.1 \mathrm{~s}$, where "s" is for indicating that it is stronger.

Theorem 6.1s Let all conditions of Theorem 6.1 hold. Then there is a permutation $\tau$ of $\{1,2, \cdots, n\}$ such that

$$
\begin{aligned}
& \sqrt{\sum_{i=1}^{n}\left[\varrho_{2}\left(\lambda_{i}, \tilde{\lambda}_{\tau(i)}\right)\right]^{2}} \leq \kappa(X) \kappa(\tilde{X}) \times \\
& \quad \min _{U \in \mathbf{U}_{n}} \min \left\{\sqrt{\left\|U-D_{1}\right\|_{\mathrm{F}}^{2}+\left\|U^{*}-D_{2}^{-1}\right\|_{\mathrm{F}}^{2}}, \sqrt{\left\|U^{*}-D_{1}^{-1}\right\|_{\mathrm{F}}^{2}+\left\|U-D_{2}\right\|_{\mathrm{F}}^{2}}\right\} .
\end{aligned}
$$

Suppose now $A \in \mathbf{C}^{n}$ is an normal matrix, i.e., $A^{*} A=A A^{*}$, and perturb $A$ to $\tilde{A}=D_{1}^{*} A D_{2}$. The question is: When is $\tilde{A}$ also normal? This is a rather interesting question, and an instant answer is that $\tilde{A}$ is normal provided

$$
D_{2}^{*} A^{*} D_{1} D_{1}^{*} A D_{2}=D_{1}^{*} A D_{2} D_{2}^{*} A^{*} D_{1} .
$$

However, this condition is, perhaps, too general to be useful. I do not know how to approach this problem yet and therefore this question will not be addressed further in what follows. On the other hand, if we happen to know that $\tilde{A}$ is also normal, the following theorem, as a corollary of Theorem $6.1 \mathrm{~s}$, indicates that the eigenvalues of $A$ and $\widetilde{A}$ agree to high relative accuracy.

Theorem 6.2 Let $A$ and $\widetilde{A}=D_{1}^{*} A D_{2}$ be two $n \times n$ normal matrices with eigenvalues (2.1), where $D_{1}$ and $D_{2}$ are nonsingular. Then there is a permutation $\tau$ of $\{1,2, \cdots, n\}$ such that

$$
\begin{aligned}
& \sqrt{\sum_{i=1}^{n}\left[\varrho_{2}\left(\lambda_{i}, \tilde{\lambda}_{\tau(i)}\right)\right]^{2}} \\
& \leq \min _{U \in \mathbf{U}_{n}} \min \left\{\sqrt{\left\|U-D_{1}\right\|_{\mathrm{F}}^{2}+\left\|U^{*}-D_{2}^{-1}\right\|_{\mathrm{F}}^{2}}, \sqrt{\left\|U^{*}-D_{1}^{-1}\right\|_{\mathrm{F}}^{2}+\left\|U-D_{2}\right\|_{\mathrm{F}}^{2}}\right\} .
\end{aligned}
$$


Generally we do not know how to relate the upper bound in (6.3) to the singular values of $D_{1}$ and $D_{2}$, unless further information on $D_{1}$ and $D_{2}$ is available. In the case of $D_{1}=D_{2}=D$, there is a simple solution. In fact, we can solve easily the following minimization problem: find a $U_{0} \in \mathbf{U}_{n}$ such that for any unitarily invariant norm $\|\cdot\|$

$$
\min _{U \in \mathbf{U}_{n}}\|U-D\|=\left\|U_{0}-D\right\| \quad \text { and } \min _{U \in \mathbf{U}_{n}}\left\|U^{*}-D^{-1}\right\|\|=\| U_{0}^{*}-D^{-1}\|\|
$$

in terms of $S V D$ of $D$. As a matter of fact, let SVD of $D$ be given by (5.3). It follows from Theorem 4.7 that

$$
\|U-D\| \geq\left\|I-\Sigma_{\mathrm{d}}\right\| \quad \text { and } \quad\left\|U^{*}-D^{-1}\right\| \geq \geq\left\|I-\Sigma_{\mathrm{d}}^{-1}\right\| .
$$

On the other hand, there is one $U_{0} \stackrel{\text { def }}{=} U_{\mathrm{d}} V_{\mathrm{d}}^{*}$ which realizes the two equality. Now applying Theorem 6.2 to Hermitian matrices leads to

Theorem 6.3 Let $A$ and $\tilde{A}=D^{*} A D$ be two $n \times n$ Hermitian matrices with eigenvalues (2.1), where $D$ is nonsingular. Then there is a permutation $\tau$ of $\{1,2, \cdots, n\}$ such that

$$
\sqrt{\sum_{i=1}^{n}\left[\varrho_{2}\left(\lambda_{i}, \tilde{\lambda}_{\tau(i)}\right)\right]^{2}} \leq \min _{U \in \mathbf{U}_{n}} \sqrt{\|U-D\|_{\mathrm{F}}^{2}+\left\|U^{*}-D^{-1}\right\|_{\mathrm{F}}^{2}}=\sqrt{\left\|I-\Sigma_{\mathbf{d}}\right\|_{\mathrm{F}}^{2}+\left\|I-\Sigma_{\mathbf{d}}^{-1}\right\|_{\mathrm{F}}^{2}} .
$$

It is worth mentioning that the permutation $\tau$ in Theorem 6.3 may not be the identity permutation, assuming eigenvalues are ordered descendingly as in (2.2). However, one can always choose a $\tau$ such that eigenvalues of the same sign are paired to each other and zero eigenvalues to zero eigenvalues. (See Proposition 3.6.) A comparison of this theorem and the inequality (5.2) in Theorem 5.1 leads to the following conclusions:

1. Theorem 6.3 covers both the definite case and the indefinite case, while the inequality (5.2) in Theorem 5.1 is for the definite case only.

2. When applying to the definite case, (5.2) is sharper than (6.6). As a matter of fact, (6.6) is a corollary of (5.2) in this case. In fact, if $A$ is nonnegative definite

$$
\begin{aligned}
\sqrt{\sum_{i=1}^{n}\left[\varrho_{2}\left(\lambda_{i}, \tilde{\lambda}_{i}\right)\right]^{2}} & \leq \frac{1}{\sqrt{2}} \sqrt{\sum_{i=1}^{n}\left[\chi\left(\lambda_{i}, \tilde{\lambda}_{i}\right)\right]^{2}} & & \text { (by Proposition 3.11) } \\
& \leq \frac{1}{\sqrt{2}}\left\|\Sigma_{\mathbf{d}}-\Sigma_{\mathrm{d}}^{-1}\right\|_{\mathrm{F}} & & \text { (by }(5.2)) \\
& \leq \sqrt{\left\|I-\Sigma_{\mathrm{d}}\right\|_{\mathrm{F}}^{2}+\left\|I-\Sigma_{\mathrm{d}}^{-1}\right\|_{\mathrm{F}}^{2}} . & & \text { (by Lemma 6.1 below) }
\end{aligned}
$$

\section{Lemma 6.1}

$$
\frac{1}{\sqrt{2}}\left\|\Sigma_{\mathrm{d}}-\Sigma_{\mathrm{d}}^{-1}\right\|_{\mathrm{F}} \leq \sqrt{\left\|I-\Sigma_{\mathrm{d}}\right\|_{\mathrm{F}}^{2}+\left\|I-\Sigma_{\mathrm{d}}^{-1}\right\|_{\mathrm{F}}^{2}}
$$

and the equality holds if and only if $\Sigma_{\mathrm{d}}=I$, i.e., $D$ is unitary. 
Proof: Notice that for $\xi \in \mathbf{R}$

$$
\left|\xi-\frac{1}{\xi}\right| \leq\left|\xi-1+1-\frac{1}{\xi}\right| \leq|\xi-1|+\left|1-\frac{1}{\xi}\right| \leq \sqrt{2} \sqrt{|\xi-1|^{2}+\left|1-\frac{1}{\xi}\right|^{2}}
$$

and the equality sign holds if and only if $\xi=1$.

The theorem below is a generalization of Theorems 4.3 and 4.4 for the spectral norm and that of Theorem 4.5.

Theorem 6.4 To the hypotheses of Theorem 6.1 add this: all $\lambda_{i}$ 's and $\tilde{\lambda}_{j}$ 's are nonnegative and are arranged descendingly as described in (2.2). Then we have

$$
\begin{aligned}
& \max _{1 \leq i \leq n} \varrho_{p}\left(\lambda_{i}, \tilde{\lambda}_{i}\right) \leq \kappa_{r}(X) \kappa_{r}(\tilde{X}) \times \\
& \quad \min \left\{\sqrt[q]{\left\|I-D_{1}^{*}\right\|_{r}^{q}+\left\|I-D_{2}^{-1}\right\|_{r}^{q}}, \sqrt[q]{\left\|I-D_{1}^{-*}\right\|_{r}^{q}+\left\|I-D_{2}\right\|_{r}^{q}}\right\},
\end{aligned}
$$

where $1 \leq r \leq \infty$ and $q=p /(p-1)$.

Similarly to Theorem 6.1 , there is a stronger version of this theorem for $r=2$ as follows.

Theorem 6.4s Let all conditions of Theorem 6.4 hold. Then

$$
\begin{aligned}
& \max _{1 \leq i \leq n} \varrho_{p}\left(\lambda_{i}, \tilde{\lambda}_{i}\right) \leq \kappa(X) \kappa(\tilde{X}) \times \\
& \min _{U \in \mathbf{U}_{n}} \min \left\{\sqrt[q]{\left\|U-D_{1}\right\|_{2}^{q}+\left\|U^{*}-D_{2}^{-1}\right\|_{2}^{q}}, \sqrt[q]{\left\|U^{*}-D_{1}^{-1}\right\|_{2}^{q}+\left\|U-D_{2}\right\|_{2}^{q}}\right\}
\end{aligned}
$$

where $q=p /(p-1)$.

As a consequence of this theorem and our solution to the optimization problem (6.4), we deduce that

Theorem 6.5 Under the conditions of Theorem 6.3, if $A$ is nonnegative definite and the eigenvalues of $A$ and $\tilde{A}$ are ordered descendingly as in (2.2), then

$$
\max _{1 \leq i \leq n} \varrho_{p}\left(\lambda_{i}, \tilde{\lambda}_{i}\right)=\sqrt[q]{\left\|I-\Sigma_{\mathbf{d}}\right\|_{2}^{q}+\left\|I-\Sigma_{\mathbf{d}}^{-1}\right\|_{2}^{q}}
$$

where $\Sigma_{\mathbf{d}}$ is defined in (5.3) and $q=p /(p-1)$.

However, Theorem 6.5 is superseded by Theorem 5.1. To see this, we notice that

1. Both Theorem 5.1 and Theorem 6.5 work for the nonnegative definite case.

2. (6.9) can be deduced from (5.1).

In fact, (5.1) and Proposition 3.11 imply that

$$
\max _{1 \leq i \leq n} \varrho_{p}\left(\lambda_{i}, \tilde{\lambda}_{i}\right) \leq 2^{-1 / p} \chi\left(\lambda_{i}, \tilde{\lambda}_{i}\right) \leq 2^{-1 / p}\left\|\Sigma_{\mathbf{d}}-\Sigma_{\mathbf{d}}^{-1}\right\|_{2} \leq \sqrt[q]{\left\|I-\Sigma_{\mathbf{d}}\right\|_{2}^{q}+\left\|I-\Sigma_{\mathbf{d}}^{-1}\right\|_{2}^{q}},
$$

by Lemma 6.2 below. But still (6.9) looks nice and clean. 


\section{Lemma 6.2}

$$
\left\|\Sigma_{\mathbf{d}}-\Sigma_{\mathrm{d}}^{-1}\right\|_{2} \leq 2^{1 / p} \sqrt[q]{\left\|I-\Sigma_{\mathrm{d}}\right\|_{2}^{q}+\left\|I-\Sigma_{\mathbf{d}}^{-1}\right\|_{2}^{q}},
$$

and the equality holds if and only if $\Sigma_{\mathrm{d}}=I$, i.e., $D$ is unitary.

Proof: Let $\xi \in \sigma(D)$ be the one such that $\left\|\Sigma_{\mathbf{d}}-\Sigma_{\mathbf{d}}^{-1}\right\|_{2}=\left|\xi-\frac{1}{\xi}\right|$. Then

$$
\begin{aligned}
\left\|\Sigma_{\mathbf{d}}-\Sigma_{\mathbf{d}}^{-1}\right\|_{2} & =\left|\xi-\frac{1}{\xi}\right| \leq|\xi-1|+\left|1-\frac{1}{\xi}\right| \\
& \leq 2^{1 / p} \sqrt[q]{|\xi-1|^{q}+\left|1-\frac{1}{\xi}\right|^{q}} \\
& \leq 2^{1 / p} \sqrt[q]{|| I-\left.\Sigma_{\mathbf{d}}\right|_{2} ^{q}+\left\|I-\Sigma_{\mathbf{d}}^{-1}\right\|_{2}^{q}},
\end{aligned}
$$

as required.

So far we have considered the case when both $A$ and $\tilde{A}$ are diagonalizable. In what follows, we weaken this assumption by requiring only $A$ to be diagonalizable and derive a relative eigenvalue perturbation bound of Bauer-Fike Type [2].

Theorem 6.6 Assume that $A \in \mathbf{C}^{n \times n}$ is diagonalizable and admits the following decomposition

$$
A=X \Lambda X^{-1} \text { where } \Lambda=\operatorname{diag}\left(\lambda_{1}, \cdots, \lambda_{n}\right) .
$$

Assume 5 also either $\tilde{A}=D A$ or $\tilde{A}=A D$. Then for any $\tilde{\lambda} \in \lambda(\tilde{A})$ there exists a $\lambda \in \lambda(A)$ such that

$$
\min _{\lambda \in \lambda(A)} \frac{|\tilde{\lambda}-\lambda|}{|\lambda|} \leq\left\|X^{-1}(D-I) X\right\|_{p} \leq \kappa_{p}(X)\|I-D\|_{p}
$$

\section{A Theorem of Ostrowski and Other Developments}

In this section, we briefly review the current state of research on the problems listed in $\$ 1.1$ and present our remarks.

Let $A$ be an $n \times n$ Hermitian matrix. Perturbing $A$ to $D^{*} A D$, where $D$ is nonsingular, is actually performing a congruence transformation to $A$ by $D$. The following theorem is due to Ostrowski $[32,1959]$ (see also [17, pp.224-225]).

Theorem 7.1 (Ostrowski) Let $A$ and $\tilde{A}=D^{*} A D$ be two $n \times n$ Hermitian matrices with eigenvalues (2.1) ordered descendingly as in (2.2), where $D$ is nonsingular. Then there exist $\theta_{j}$ 's so that

$$
\sigma_{\min }(D)^{2} \leq \theta_{j} \leq \sigma_{\max }(D)^{2} \quad \text { and } \quad \tilde{\lambda}_{j}=\theta_{j} \lambda_{j} \quad \text { for } 1 \leq j \leq n
$$

\footnotetext{
${ }^{5}$ Unlike in our previous theorems, here we do not have to assume that $D$ is nonsingular. Of course, if $D$ is far away from $I$, the bound (6.12) does not tell us much; if $D$ is close enough to $I$, it has to be nonsingular.
} 
Ostrowski's theorem implies immediately a relative perturbation bound on Hermitian eigenvalues.

Theorem 7.2 Let the conditions of Theorem 7.1 hold. Then

$$
\frac{\left|\tilde{\lambda}_{j}-\lambda_{j}\right|}{\left|\lambda_{j}\right|} \leq\left\|I-D^{*} D\right\|_{2} \quad \text { for } 1 \leq j \leq n
$$

or in other words,

$$
\tilde{\lambda}_{j}=\lambda_{j}\left(1+\delta_{j}\right) \quad \text { with } \quad\left|\delta_{j}\right| \leq\left\|I-D^{*} D\right\|_{2} \quad \text { for } 1 \leq j \leq n .
$$

Inequality (5.1) of Theorem 5.1 and Theorem 7.2 are independent in the sense that one can not be inferred from the other; but Theorem 7.2 covers more while Theorem 5.1 covers nonnegative definite matrices only.

Ostrowski's theorem also applies to singular value variations for matrices $B$ and $\widetilde{B}=$ $D_{1}^{*} B D$ by working with Hermitian matrices

$$
\left(\begin{array}{cc} 
& B^{*} \\
B &
\end{array}\right) \text { and }\left(\begin{array}{cc} 
& \widetilde{B}^{*} \\
\widetilde{B} &
\end{array}\right)=\left(\begin{array}{cc}
D_{2} & \\
& D_{1}
\end{array}\right)^{*}\left(\begin{array}{cc} 
& B^{*} \\
B &
\end{array}\right)\left(\begin{array}{cc}
D_{2} & \\
& D_{1}
\end{array}\right) .
$$

Given the singular values (2.3) of $B$ and $\widetilde{B}$, it is known that besides $m-n$ (if $m>n$ ) zero eigenvalues, the eigenvalues of the two matrices in (7.1) are $\pm \sigma_{i}$, and $\pm \tilde{\sigma}_{i}$, respectively.

Corollary 7.1 Let $B$ and $\widetilde{B}=D_{1}^{*} B D_{2}$ be two $m \times n$ matrices with singular values (2.3) ordered descendingly as in (2.4), where $D_{1}$ and $D_{2}$ are nonsingular. Then

$$
\min \left\{\sigma_{\min }\left(D_{1}\right)^{2}, \sigma_{\min }\left(D_{2}\right)^{2}\right\} \leq \frac{\tilde{\sigma}_{j}}{\sigma_{j}} \leq \max \left\{\sigma_{\max }\left(D_{1}\right)^{2}, \sigma_{\max }\left(D_{2}\right)^{2}\right\} \quad \text { for } 1 \leq j \leq n
$$

which gives

$$
\frac{\left|\tilde{\sigma}_{j}-\sigma_{j}\right|}{\sigma_{j}} \leq \max \left\{\left\|I-D_{1}^{*} D_{1}\right\|_{2},\left\|I-D_{2}^{*} D_{2}\right\|_{2}\right\} \quad \text { for } 1 \leq j \leq n,
$$

or in other words,

$$
\tilde{\sigma}_{j}=\sigma_{j}\left(1+\gamma_{j}\right) \quad \text { with } \quad\left|\gamma_{j}\right| \leq \max \left\{\left\|I-D_{1}^{*} D_{1}\right\|_{2},\left\|I-D_{2}^{*} D_{2}\right\|_{2}\right\} \quad \text { for } 1 \leq j \leq n .
$$

This corollary, though, an immediate consequence of the above Ostrowski's theorem and the equation (7.1), has appeared no where. It turns out that Corollary 7.1 provides a less sharp bound than the following theorem due to Eisenstat and Ipsen [10, 1993]. It can also be derived from Ostrowski's theorem.

Theorem 7.3 (Eisenstat-Ipsen) Under the conditions of Corollary 7.1, we have

$$
\sigma_{\min }\left(D_{1}\right) \sigma_{\min }\left(D_{2}\right) \leq \frac{\tilde{\sigma}_{j}}{\sigma_{j}} \leq \sigma_{\max }\left(D_{1}\right) \sigma_{\max }\left(D_{2}\right) \quad \text { for } 1 \leq j \leq n
$$


which yields

$$
\frac{\left|\tilde{\sigma}_{j}-\sigma_{j}\right|}{\sigma_{j}} \leq \max \left\{\left|1-\sigma_{\min }\left(D_{1}\right) \sigma_{\min }\left(D_{2}\right)\right|,\left|1-\sigma_{\max }\left(D_{1}\right) \sigma_{\max }\left(D_{2}\right)\right|\right\} \quad \text { for } 1 \leq j \leq n,
$$

or in other words, $\tilde{\sigma}_{j}=\sigma_{j}\left(1+\gamma_{j}\right)$ with

$$
\left|\gamma_{j}\right| \leq \max \left\{\left|1-\sigma_{\min }\left(D_{1}\right) \sigma_{\min }\left(D_{2}\right)\right|,\left|1-\sigma_{\max }\left(D_{1}\right) \sigma_{\max }\left(D_{2}\right)\right|\right\},
$$

for $1 \leq j \leq n$.

Theorem 7.3 always provides a sharper bound than Corollary 7.1 does, as the following lemma indicates.

Lemma 7.1 For $\xi, \zeta \geq 0$,

$$
\max \left\{\left|1-\xi^{2}\right|,\left|1-\zeta^{2}\right|\right\} \geq|1-\xi \zeta|,
$$

and the equality holds if and only if $\xi=\zeta$.

Proof: The inequality is obvious if either $\max \{\xi, \zeta\} \leq 1$ or $\min \{\xi, \zeta\} \geq 1$. It is also clearly true if either $\xi=1$ or $\zeta=1$. Now it suffices for us to consider the case when $0 \leq \xi<1<\zeta$. There are two subcases: $\zeta^{2}-1 \leq 1-\xi^{2}$ or $\zeta^{2}-1>1-\xi^{2}$.

1. $\zeta^{2}-1 \leq 1-\xi^{2} \Rightarrow \xi^{2}+\zeta^{2} \leq 2 \Rightarrow 2 \xi \zeta<\xi^{2}+\zeta^{2} \leq 2$ (by the Cauchy-Schwarz inequality and since $\xi \neq \zeta) \Rightarrow \xi \zeta<1$. Now notice that

$$
\xi^{2}<\xi \zeta \Rightarrow 1-\xi^{2}>1-\xi \zeta=|1-\xi \zeta| \text {. }
$$

2. $\zeta^{2}-1>1-\xi^{2} \Rightarrow \xi^{2}+\zeta^{2}>2 \Rightarrow \xi \zeta+\zeta^{2} \geq \xi^{2}+\zeta^{2}>2 \Rightarrow \zeta^{2}-1>1-\xi \zeta$; on the other hand, $\zeta^{2}>\xi \zeta \Rightarrow \zeta^{2}-1>\xi \zeta-1$. So

$$
\zeta^{2}-1>\max \{1-\xi \zeta, \xi \zeta-1\}=|1-\xi \zeta| \text {. }
$$

From the above proof, it is clear that $\max \left\{\left|1-\xi^{2}\right|,\left|1-\zeta^{2}\right|\right\}=|1-\xi \zeta|$ cannot happen when $0 \leq \xi<1<\zeta$; it is not hard to see when $\max \{\xi, \zeta\} \leq 1$ or $\min \{\xi, \zeta\} \geq 1$, the equality cannot happen, either, unless $\xi=\zeta$.

Regarding to graded matrices, the following two theorems are due to Demmel and Veselić [9, 1992] and Mathias [29, 1994].

Theorem 7.4 (Demmel-Veselić) Under the conditions of Theorem 5.4, we have

$$
\frac{\left|\widetilde{\lambda}_{j}-\lambda_{j}\right|}{\left|\lambda_{j}\right|} \leq\left\|H^{-1}\right\|_{2}\|\Delta H\|_{2} \quad \text { for } 1 \leq j \leq n,
$$

or in other words,

$$
\tilde{\lambda}_{j}=\lambda_{j}\left(1+\delta_{j}\right) \quad \text { with } \quad\left|\delta_{j}\right| \leq\left\|H^{-1}\right\|_{2}\|\Delta H\|_{2} \quad \text { for } 1 \leq j \leq n .
$$


Theorem 7.5 (Mathias) Under the conditions of Theorem 5.5, we have

$$
\frac{\left|\widetilde{\sigma}_{j}-\sigma_{j}\right|}{\sigma_{j}} \leq\left\|G^{-1}\right\|_{2}\|\Delta G\|_{2} \quad \text { for } 1 \leq j \leq n
$$

or in other words,

$$
\tilde{\sigma}_{j}=\sigma_{j}\left(1+\gamma_{j}\right) \quad \text { with } \quad\left|\gamma_{j}\right| \leq\left\|G^{-1}\right\|_{2}\|\Delta G\|_{2} \quad \text { for } 1 \leq j \leq n .
$$

Finally, let us see what we can get from Theorems 7.2, 7.4, 7.5 and 7.3 and Corollary 7.1 , in terms of the two kinds of relative distances defined in $\S 3$.

1. From Theorem 7.2 , we have for $1 \leq j \leq n$

$$
\begin{aligned}
\varrho_{p}\left(\lambda_{j}, \tilde{\lambda}_{j}\right) \leq \varrho_{\infty}\left(\lambda_{j}, \tilde{\lambda}_{j}\right) & \leq\left\|I-D^{*} D\right\|_{2} \\
\chi\left(\lambda_{j}, \tilde{\lambda}_{j}\right) & \leq \frac{\left\|I-D^{*} D\right\|_{2}}{\sigma_{\min }(D)} .
\end{aligned}
$$

The inequality (7.3) holds because

$$
\varrho_{\infty}\left(\lambda_{j}, \tilde{\lambda}_{j}\right)=\frac{\left|\tilde{\lambda}_{j}-\lambda_{j}\right|}{\max \left\{\left|\lambda_{j}\right|,\left|\tilde{\lambda}_{j}\right|\right\}} \leq \frac{\left|\tilde{\lambda}_{j}-\lambda_{j}\right|}{\left|\lambda_{j}\right|} \leq\left\|I-D^{*} D\right\|_{2} ;
$$

and the inequality (7.4) holds because

$$
\chi\left(\lambda_{j}, \tilde{\lambda}_{j}\right)=\frac{\left|\tilde{\lambda}_{j}-\lambda_{j}\right|}{\sqrt{\left|\lambda_{j}\right|\left|\tilde{\lambda}_{j}\right|}}=\frac{\left|\tilde{\lambda}_{j}-\lambda_{j}\right|}{\left|\lambda_{j}\right|} \sqrt{\frac{\left|\lambda_{j}\right|}{\left|\widetilde{\lambda}_{j}\right|}} \leq \frac{\left\|I-D^{*} D\right\|_{2}}{\sigma_{\min }(D)} .
$$

2. From Corollary 7.1 and by similar reasonings above, we have for $1 \leq j \leq n$

$$
\begin{aligned}
\varrho_{\infty}\left(\sigma_{j}, \widetilde{\sigma}_{j}\right) & \leq \max \left\{\left\|I-D_{1}^{*} D_{1}\right\|_{2},\left\|I-D_{2}^{*} D_{2}\right\|_{2}\right\} \\
\chi\left(\sigma_{j}, \widetilde{\sigma}_{j}\right) & \leq \frac{\max \left\{\left\|I-D_{1}^{*} D_{1}\right\|_{2},\left\|I-D_{2}^{*} D_{2}\right\|_{2}\right\}}{\min \left\{\sigma_{\min }\left(D_{1}\right), \sigma_{\min }\left(D_{2}\right)\right\}} .
\end{aligned}
$$

3. From Theorem 7.3 , we have for $1 \leq j \leq n$

$$
\begin{aligned}
\varrho_{\infty}\left(\sigma_{j}, \tilde{\sigma}_{j}\right) & \leq \max \left\{\left|1-\sigma_{\min }\left(D_{1}\right) \sigma_{\min }\left(D_{2}\right)\right|,\left|1-\sigma_{\max }\left(D_{1}\right) \sigma_{\max }\left(D_{2}\right)\right|\right\} \\
\chi\left(\sigma_{j}, \widetilde{\sigma}_{j}\right) & \leq \frac{\max \left\{\left|1-\sigma_{\min }\left(D_{1}\right) \sigma_{\min }\left(D_{2}\right)\right|,\left|1-\sigma_{\max }\left(D_{1}\right) \sigma_{\max }\left(D_{2}\right)\right|\right\}}{\sqrt{\sigma_{\min }\left(D_{1}\right) \sigma_{\min }\left(D_{2}\right)}} .
\end{aligned}
$$

Inequalities (7.7) and (7.8) are sharper than (7.5) and (7.6), respectively.

4. From Theorem 7.4 , we have for $1 \leq j \leq n$

$$
\begin{aligned}
\varrho_{\infty}\left(\lambda_{j}, \tilde{\lambda}_{j}\right) & \leq\left\|H^{-1}\right\|_{2}\|\Delta H\|_{2}, \\
\chi\left(\lambda_{j}, \tilde{\lambda}_{j}\right) & \leq \frac{\left\|H^{-1}\right\|_{2}\|\Delta H\|_{2}}{\sqrt{1-\left\|H^{-1}\right\|_{2}\|\Delta H\|_{2}}} .
\end{aligned}
$$

Inequality (7.10) has been derived in Theorem 5.4. 
5. From Theorem 7.5, it follows for $1 \leq j \leq n$

$$
\begin{aligned}
\varrho_{\infty}\left(\sigma_{j}, \tilde{\sigma}_{j}\right) & \leq\left\|G^{-1}\right\|_{2}\|\Delta G\|_{2}, \\
\chi\left(\sigma_{j}, \tilde{\sigma}_{j}\right) & \leq \frac{\left\|G^{-1}\right\|_{2}\|\Delta G\|_{2}}{\sqrt{1-\left\|G^{-1}\right\|_{2}\|\Delta G\|_{2}}} .
\end{aligned}
$$

Inequality (7.12) turns out to be sharper than the last " $\leq$ " in (5.13) of Theorem 5.5 , but not the first two.

\section{Proofs of Theorems 6.1 and 6.4}

To prove the theorems, we need a little preparation. A matrix $Y=\left(y_{i j}\right) \in \mathbf{R}^{n \times n}$ is doubly stochastic if all $y_{i j} \geq 0$ and

$$
\sum_{k=1}^{n} y_{i k}=\sum_{k=1}^{n} y_{k j}=1 \quad \text { for } i, j=1,2, \cdots, n .
$$

A matrix $P \in \mathbf{R}^{n \times n}$ is called a permutation matrix if exactly one entry in each row and each column equals to 1 and all others are zero. Let $e_{i}$ be the $i$ th column vector of $I_{n}$. Each permutation matrix $P$ corresponds to a unique permutation $\tau$ of $\{1,2, \cdots, n\}$ in such a way:

$$
P=\left(e_{\tau(1)}, e_{\tau(2)}, \cdots, e_{\tau(n)}\right),
$$

and vice versa. Thus there are $n$ ! permutation matrices. The following wonderful result is due to Birkhoff $[5,1946]$ (see also [17, pp.527-528]).

Lemma 8.1 (Birkhoff) An $n \times n$ matrix is doubly stochastic if and only if it lies in the convex hull of permutation matrices.

Lemma 8.2 Let $Y=\left(y_{i j}\right)$ be an $n \times n$ doubly stochastic matrix, and let $M=\left(m_{i j}\right) \in$ $\mathbf{C}^{n \times n}$. Then there exists a permutation $\tau$ of $\{1,2, \cdots, n\}$ such that

$$
\sum_{i, j=1}^{n}\left|m_{i j}\right|^{2} y_{i j} \geq \sum_{i=1}^{n}\left|m_{i \tau(i)}\right|^{2}
$$

Proof: Denote all $n \times n$ permutation matrices as $P_{k}$, and their corresponding permutations of $\{1,2, \cdots, n\}$ as $\tau_{k}$, where $k=1,2, \cdots, n !$. It follows from Lemma 8.1 that $Y$ can be written as $Y=\sum_{k=1}^{n !} \alpha_{k} P_{k}$, where $\alpha_{k} \geq 0$ and $\sum_{k=1}^{n !} \alpha_{k}=1$. Hence

$$
\sum_{i, j=1}^{n}\left|m_{i j}\right|^{2} y_{i j}=\sum_{k=1}^{n !} \alpha_{k} \sum_{i=1}^{n}\left|m_{i \tau_{k}(i)}\right|^{2} \geq \min _{1 \leq k \leq n !} \sum_{i=1}^{n}\left|m_{i \tau_{k}(i)}\right|^{2},
$$

as was to be shown. 
The technique in the above proof is quite standard. It was first used by Hoffman and Wielandt $[16,1953]$ to prove Theorem 4.1, and later by Sun [36, 1982] to prove a Hoffman-Wielandt type theorem for a special class of matrix pencils (and by maybe many others).

The following lemma is due to Elsner and Friedland [12, 1995].

Lemma 8.3 (Elsner-Friedland) Let $Y=\left(y_{i j}\right) \in \mathbf{C}^{n \times n}$. Then there exist two $n \times n$ doubly stochastic matrices $Y_{1}, Y_{2}$ such that entrywisely

$$
\sigma_{\min }(Y)^{2} Y_{1} \leq\left(\left|y_{i j}\right|^{2}\right) \leq \sigma_{\max }(Y)^{2} Y_{2}
$$

Proof of Theorem 6.1: Let us first derive our perturbation equations.

$$
A-\tilde{A}=A-D_{1}^{*} A D_{2}=A-A D_{2}+A D_{2}-D_{1}^{*} A D_{2}=A\left(I-D_{2}\right)+\left(D_{1}^{-*}-I\right) \tilde{A} .
$$

Pre- and post-multiply the equations by $X^{-1}$ and $\tilde{X}$, respectively, to get

$$
\Lambda X^{-1} \tilde{X}-X^{-1} \tilde{X} \tilde{\Lambda}=\Lambda X^{-1}\left(I-D_{2}\right) \tilde{X}+X^{-1}\left(D_{1}^{-*}-I\right) \tilde{X} \tilde{\Lambda} .
$$

Set

$$
Y \stackrel{\text { def }}{=} X^{-1} \tilde{X}=\left(y_{i j}\right), E \stackrel{\text { def }}{=} X^{-1}\left(I-D_{2}\right) \tilde{X}=\left(e_{i j}\right), \tilde{E} \stackrel{\text { def }}{=} X^{-1}\left(D_{1}^{-*}-I\right) \tilde{X}=\left(\widetilde{e}_{i j}\right) .
$$

Then equation (8.1) reads $\Lambda Y-Y \tilde{\Lambda}=\Lambda E+\tilde{E} \tilde{\Lambda}$, or componentwise $\lambda_{i} y_{i j}-y_{i j} \tilde{\lambda}_{j}=$ $\lambda_{i} e_{i j}+\widetilde{e}_{i j} \tilde{\lambda}_{j}$, so

$$
\left(\left|\lambda_{i}\right|^{2}+\left|\tilde{\lambda}_{j}\right|^{2}\right)\left(\left|e_{i j}\right|^{2}+\left|\widetilde{e}_{i j}\right|^{2}\right) \geq\left|\lambda_{i} e_{i j}+\widetilde{e}_{i j} \tilde{\lambda}_{j}\right|=\left|\left(\lambda_{i}-\tilde{\lambda}_{j}\right) y_{i j}\right|^{2},
$$

which yields $\left|e_{i j}\right|^{2}+\left|\widetilde{e}_{i j}\right|^{2} \geq\left[\varrho_{2}\left(\lambda_{i}, \tilde{\lambda}_{j}\right)\right]^{2}\left|y_{i j}\right|^{2}$. Hence

$$
\left\|X^{-1}\left(I-D_{2}\right) \tilde{X}\right\|_{\mathrm{F}}^{2}+\left\|X^{-1}\left(D_{1}^{-*}-I\right) \tilde{X}\right\|_{\mathrm{F}}^{2} \geq \sum_{i, j=1}^{n}\left[\varrho_{2}\left(\lambda_{i}, \tilde{\lambda}_{j}\right)\right]^{2}\left|y_{i j}\right|^{2} .
$$

Inequality (8.2), Lemmas 8.2 and 8.3 imply that

$$
\left\|X^{-1}\left(I-D_{2}\right) \tilde{X}\right\|_{\mathrm{F}}^{2}+\left\|X^{-1}\left(D_{1}^{-*}-I\right) \tilde{X}\right\|_{\mathrm{F}}^{2} \geq \sigma_{\min }(Y)^{2} \sum_{i=1}^{n}\left[\varrho_{2}\left(\lambda_{i}, \tilde{\lambda}_{\tau(i)}\right)\right]^{2}
$$

for some permutation $\tau$ of $\{1,2, \cdots, n\}$. Since

$$
\sigma_{\min }(Y)=\left\|Y^{-1}\right\|_{2}^{-1}=\left\|\tilde{X}^{-1} X\right\|_{2}^{-1} \geq\left\|\tilde{X}^{-1}\right\|_{2}^{-1}\|X\|_{2}^{-1},
$$

we have

$$
\begin{aligned}
& \left\|\tilde{X}^{-1}\right\|_{2}\|X\|_{2} \sqrt{\left\|X^{-1}\left(I-D_{2}\right) \tilde{X}\right\|_{\mathrm{F}}^{2}+\left\|X^{-1}\left(D_{1}^{-*}-I\right) \tilde{X}\right\|_{\mathrm{F}}^{2}} \\
& \quad \geq\left\|\tilde{X}^{-1}\right\|_{2}\|X\|_{2} \sigma_{\min }(Y) \sqrt{\sum_{i=1}^{n}\left[\varrho_{2}\left(\lambda_{i}, \tilde{\lambda}_{\tau(i)}\right)\right]^{2}} \geq \sqrt{\sum_{i=1}^{n}\left[\varrho_{2}\left(\lambda_{i}, \tilde{\lambda}_{\tau(i)}\right)\right]^{2}} .
\end{aligned}
$$


On the other hand, we have

$$
A-\widetilde{A}=A-D_{1}^{*} A D_{2}=A-D_{1}^{*} A+D_{1}^{*} A-D_{1}^{*} A D_{2}=\left(I-D_{1}^{*}\right) A+\widetilde{A}\left(D_{2}^{-1}-I\right) .
$$

Pre- and post-multiply the equations by $\widetilde{X}^{-1}$ and $X$, respectively, to get

$$
\tilde{X}^{-1} X \Lambda-\tilde{\Lambda} \widetilde{X}^{-1} X=\tilde{X}^{-1}\left(I-D_{1}^{*}\right) X \Lambda+\widetilde{\Lambda} \widetilde{X}^{-1}\left(D_{2}^{-1}-I\right) X .
$$

Set $\tilde{Y} \stackrel{\text { def }}{=} \tilde{X}^{-1} X=\left(\tilde{y}_{i j}\right)$. Similarly, we have

$$
\left\|\tilde{X}^{-1}\left(I-D_{1}^{*}\right) X\right\|_{\mathrm{F}}^{2}+\left\|\tilde{X}^{-1}\left(D_{2}^{-1}-I\right) X\right\|_{\mathrm{F}}^{2} \geq \sum_{i, j=1}^{n}\left[\varrho_{2}\left(\lambda_{i}, \tilde{\lambda}_{j}\right)\right]^{2}\left|\tilde{y}_{j i}\right|^{2} .
$$

Inequality (8.5), Lemmas 8.2 and 8.3 imply that

$$
\left\|\tilde{X}^{-1}\left(I-D_{1}^{*}\right) X\right\|_{\mathrm{F}}^{2}+\left\|\tilde{X}^{-1}\left(D_{2}^{-1}-I\right) X\right\|_{\mathrm{F}}^{2} \geq \sigma_{\min }(\tilde{Y})^{2} \sum_{i=1}^{n}\left[\varrho_{2}\left(\lambda_{i}, \tilde{\lambda}_{\tau(i)}\right)\right]^{2} .
$$

Notice now $\sigma_{\min }(\tilde{Y})=\left\|\tilde{Y}^{-1}\right\|_{2}^{-1}=\left\|X^{-1} \tilde{X}\right\|_{2}^{-1} \geq\left\|X^{-1}\right\|_{2}^{-1}\|\tilde{X}\|_{2}^{-1}$. Along the lines for proving (8.3), we obtain

$$
\left\|X^{-1}\right\|_{2}\|\tilde{X}\|_{2} \sqrt{\left\|\tilde{X}^{-1}\left(I-D_{1}^{*}\right) X\right\|_{\mathrm{F}}^{2}+\left\|\tilde{X}^{-1}\left(D_{2}^{-1}-I\right) X\right\|_{\mathrm{F}}^{2}} \geq \sqrt{\sum_{i=1}^{n}\left[\varrho_{2}\left(\lambda_{i}, \tilde{\lambda}_{\tau(i)}\right)^{2}\right.} .
$$

Inequality (6.1) is now a consequence of (8.3) and (8.6).

The proof of Theorem 6.4 below needs the following result due to Li [23, pp.207-208, 1993]. For $X \in \mathbf{C}^{m \times n}$, we introduce the following notation for a $k \times \ell$ submatrix of $X=\left(x_{i j}\right)$ :

$$
X\left(\begin{array}{c}
i_{1} \cdots i_{k} \\
j_{1} \cdots j_{\ell}
\end{array}\right) \stackrel{\text { def }}{=}\left(\begin{array}{cccc}
x_{i_{1} j_{1}} & x_{i_{1} j_{2}} & \cdots & x_{i_{1} j_{\ell}} \\
x_{i_{2} j_{1}} & x_{i_{2} j_{2}} & \cdots & x_{i_{2} j_{\ell}} \\
\vdots & \vdots & \ddots & \vdots \\
x_{i_{k} j_{1}} & x_{i_{k} j_{2}} & \cdots & x_{i_{k} j_{\ell}}
\end{array}\right),
$$

where $1 \leq i_{1}<\cdots<i_{k} \leq n$ and $1 \leq j_{1}<\cdots<j_{\ell} \leq n$.

Lemma $8.4(\mathrm{Li})$ Suppose that $X \in \mathbf{C}^{n \times n}$ is nonsingular, and $1 \leq i_{1}<\cdots<i_{k} \leq n$ and $1 \leq j_{1}<\cdots<j_{\ell} \leq n$, and $k+\ell>n$. Then $\left\|X\left(\begin{array}{c}i_{1} \cdots i_{k} \\ j_{1} \cdots j_{\ell}\end{array}\right)\right\|_{p} \geq\left\|X^{-1}\right\|_{p}^{-1}$. Moreover, if $X$ is unitary, then $\left\|X\left(\begin{array}{c}i_{1} \cdots i_{k} \\ j_{1} \cdots j_{\ell}\end{array}\right)\right\|_{2}=1$.

Proof of Theorem 6.4: Let $k$ be the index such that

$$
\eta_{p} \stackrel{\text { def }}{=} \max _{1 \leq i \leq n} \varrho_{p}\left(\lambda_{i}, \tilde{\lambda}_{i}\right)=\varrho_{p}\left(\lambda_{k}, \widetilde{\lambda}_{k}\right)
$$


If $\eta_{p}=0$, inequality (6.7) clearly holds. Assume $\eta_{p}>0$. Also assume, without loss of any generality, that

$$
\lambda_{k}>\widetilde{\lambda}_{k} \geq 0 .
$$

Partition $X, X^{-1}, \tilde{X}$ and $\tilde{X}^{-1}$ conformally as follows:

$$
X=\left(X_{1}, X_{2}\right), X^{-1}=\left(\begin{array}{c}
W_{1}^{*} \\
W_{2}^{*}
\end{array}\right), \tilde{X}=\left(\widetilde{X}_{1}, \widetilde{X}_{2}\right), \tilde{X}^{-1}=\left(\begin{array}{c}
\widetilde{W}_{1}^{*} \\
\widetilde{W}_{2}^{*}
\end{array}\right),
$$

where $X_{1}, W_{1} \in \mathbf{C}^{n \times k}$ and $\widetilde{X}_{1}, \widetilde{W}_{1} \in \mathbf{C}^{n \times(k-1)}$; and write $\Lambda=\operatorname{diag}\left(\Lambda_{1}, \Lambda_{2}\right)$ and $\tilde{\Lambda}=$ $\operatorname{diag}\left(\tilde{\Lambda}_{1}, \widetilde{\Lambda}_{2}\right)$, where $\Lambda_{1} \in \mathbf{R}^{k \times k}$ and $\widetilde{\Lambda}_{1} \in \mathbf{R}^{(k-1) \times(k-1)}$. It follows from equations (8.1) and (8.4) that

$$
\begin{aligned}
\Lambda_{1} W_{1}^{*} \widetilde{X}_{2}-W_{1}^{*} \widetilde{X}_{2} \widetilde{\Lambda}_{2}=\Lambda_{1} W_{1}^{*}\left(I-D_{2}\right) \widetilde{X}_{2}+W_{1}^{*}\left(D_{1}^{-*}-I\right) \widetilde{X}_{2} \widetilde{\Lambda}_{2} \\
\widetilde{W}_{2}^{*} X_{1} \Lambda_{1}-\widetilde{\Lambda}_{2} \widetilde{W}_{2}^{*} X_{1}=\widetilde{W}_{2}^{*}\left(I-D_{1}^{*}\right) X_{1} \Lambda_{1}+\widetilde{\Lambda}_{2} \widetilde{W}_{2}^{*}\left(D_{2}^{-1}-I\right) X_{1}
\end{aligned}
$$

which give

$$
\begin{aligned}
& W_{1}^{*} \widetilde{X}_{2}-\Lambda_{1}^{-1} W_{1}^{*} \widetilde{X}_{2} \widetilde{\Lambda}_{2}=W_{1}^{*}\left(I-D_{2}\right) \widetilde{X}_{2}+\Lambda_{1}^{-1} W_{1}^{*}\left(D_{1}^{-*}-I\right) \tilde{X}_{2} \widetilde{\Lambda}_{2}, \\
& \widetilde{W}_{2}^{*} X_{1}-\widetilde{\Lambda}_{2} \widetilde{W}_{2}^{*} X_{1} \Lambda_{1}^{-1}=\widetilde{W}_{2}^{*}\left(I-D_{1}^{*}\right) X_{1}+\widetilde{\Lambda}_{2} \widetilde{W}_{2}^{*}\left(D_{2}^{-1}-I\right) X_{1} \Lambda_{1}^{-1} .
\end{aligned}
$$

Lemma 8.4 implies for $1 \leq r \leq \infty$

$$
\begin{aligned}
& \left\|W_{1}^{*} \tilde{X}_{2}\right\|_{r} \geq\left\|\left(X^{-1} \tilde{X}\right)^{-1}\right\|_{r}^{-1} \geq\left\|\tilde{X}^{-1} X\right\|_{r}^{-1} \geq\left\|\tilde{X}^{-1}\right\|_{r}^{-1}\|X\|_{r}^{-1}, \\
& \left\|\widetilde{W}_{2}^{*} X_{1}\right\|_{r} \geq\left\|\left(\tilde{X}^{-1} X\right)^{-1}\right\|_{r}^{-1} \geq\left\|X^{-1} \tilde{X}\right\|_{r}^{-1} \geq\left\|X^{-1}\right\|_{r}^{-1}\|\tilde{X}\|_{r}^{-1},
\end{aligned}
$$

since $W_{1}^{*} \widetilde{X}_{2}$ is a $k \times(n-k+1)$ submatrix of $X^{-1} \tilde{X}$, and $\widetilde{W}_{2}^{*} X_{1}$ is a $(n-k+1) \times k$ submatrix of $\tilde{X}^{-1} X$ and $k+(n-k+1)=n+1>n$. Bearing in mind that $\left\|\Lambda_{1}^{-1}\right\|_{r}=1 / \lambda_{k}$ and $\left\|\tilde{\Lambda}_{2}\right\|_{r}=\tilde{\lambda}_{k}$, we have

$$
\begin{aligned}
\left(1-\frac{\tilde{\lambda}_{k}}{\lambda_{k}}\right)\left\|\tilde{X}^{-1}\right\|_{r}^{-1}\|X\|_{r}^{-1} & \leq\left(1-\frac{\tilde{\lambda}_{k}}{\lambda_{k}}\right)\left\|W_{1}^{*} \tilde{X}_{2}\right\|_{r} \\
& =\left\|W_{1}^{*} \tilde{X}_{2}\right\|_{r}-\left\|\Lambda_{1}^{-1}\right\|_{r}\left\|W_{1}^{*} \tilde{X}_{2}\right\|_{r}\left\|\tilde{\Lambda}_{2}\right\|_{r} \\
& \leq\left\|W_{1}^{*} \tilde{X}_{2}\right\|_{r}-\left\|\Lambda_{1}^{-1} W_{1}^{*} \tilde{X}_{2} \widetilde{\Lambda}_{2}\right\|_{r} \\
& \leq\left\|W_{1}^{*} \tilde{X}_{2}-\Lambda_{1}^{-1} W_{1}^{*} \tilde{X}_{2} \tilde{\Lambda}_{2}\right\|_{r} \\
& =\left\|W_{1}^{*}\left(I-D_{2}\right) \tilde{X}_{2}+\Lambda_{1}^{-1} W_{1}^{*}\left(D_{1}^{-*}-I\right) \tilde{X}_{2} \tilde{\Lambda}_{2}\right\|_{r} \quad \text { by } \\
& \leq\left\|W_{1}^{*}\left(I-D_{2}\right) \tilde{X}_{2}\right\|_{r}+\frac{\tilde{\lambda}_{k}}{\lambda_{k}}\left\|W_{1}^{*}\left(D_{1}^{-*}-I\right) \tilde{X}_{2}\right\|_{r} \\
& \leq\left\|W_{1}^{*}\right\|_{r}\left\|\tilde{X}_{2}\right\|_{r}\left(\left\|I-D_{2}\right\|_{r}+\frac{\tilde{\lambda}_{k}}{\lambda_{k}}\left\|D_{1}^{-*}-I\right\|_{r}\right) \\
& \leq\left\|X^{-1}\right\|_{r}\|\tilde{X}\|_{r} \sqrt[p]{1+\frac{\tilde{\lambda}_{k}^{p}}{\lambda_{k}^{p}} \sqrt[q]{\left\|I-D_{2}\right\|_{r}^{q}+\left\|I-D_{1}^{-*}\right\|_{r}^{q}}}
\end{aligned}
$$


Similarly, from (8.9) we obtain

$$
\left(1-\frac{\tilde{\lambda}_{k}}{\lambda_{k}}\right)\left\|X^{-1}\right\|_{r}^{-1}\|\tilde{X}\|_{r}^{-1} \leq\left\|\tilde{X}^{-1}\right\|_{r}\|X\|_{r} \sqrt[p]{1+\frac{\tilde{\lambda}_{k}^{p}}{\lambda_{k}^{p}}} \sqrt[q]{\left\|I-D_{2}^{-1}\right\|_{r}^{q}+\left\|I-D_{1}^{*}\right\|_{r}^{q}}
$$

Inequality (6.7) is now a consequence of above inequalities.

\section{Proofs of Theorems 5.1, 5.2, 5.3, 5.4, and 5.5}

Proof of Theorem 5.1: Since $A$ is nonnegative definite, there is a matrix $B \in \mathbf{C}^{n \times n}$ such that $A=B^{*} B$. With this $B, \widetilde{A}=D^{*} A D=D^{*} B^{*} B D=\widetilde{B}^{*} \widetilde{B}$, where $\widetilde{B}=B D$. Let SVDs of $B$ and $\widetilde{B}$ be

$$
B=U \Lambda^{1 / 2} V^{*} \text { and } \widetilde{B}=\widetilde{U} \widetilde{\Lambda}^{1 / 2} \widetilde{V}^{*},
$$

where $\Lambda^{1 / 2}=\operatorname{diag}\left(\sqrt{\lambda_{1}}, \sqrt{\lambda_{2}}, \cdots, \sqrt{\lambda_{n}}\right)$ and $\tilde{\Lambda}^{1 / 2}=\operatorname{diag}\left(\sqrt{\tilde{\lambda}_{1}}, \sqrt{\tilde{\lambda}_{2}}, \cdots, \sqrt{\tilde{\lambda}_{n}}\right)$. In what follows, we actually work with $B B^{\star}$ and $\widetilde{B} \widetilde{B}^{*}$, instead of $A=B^{\star} B$ and $\tilde{A}=\widetilde{B}^{*} \widetilde{B}$. We have

$$
\widetilde{B} \widetilde{B}^{*}-B B^{*}=\widetilde{B} D^{*} B^{*}-\widetilde{B} D^{-1} B^{*}=\widetilde{B}\left(D^{*}-D^{-1}\right) B^{*} .
$$

Pre- and post-multiply the above equations by $\tilde{U}^{*}$ and $U$, respectively, to get

$$
\widetilde{\Lambda} \widetilde{U}^{*} U-\widetilde{U}^{*} U \Lambda=\widetilde{\Lambda}^{1 / 2} \widetilde{V}^{*}\left(D^{*}-D^{-1}\right) V \Lambda^{1 / 2} .
$$

Write $Q \stackrel{\text { def }}{=} \widetilde{U}^{*} U=\left(q_{i j}\right)$. Equation $(9.1)$ implies

$$
\left\|\tilde{V}^{*}\left(D^{*}-D^{-1}\right) V\right\|_{\mathrm{F}}^{2}=\left\|D^{*}-D^{-1}\right\|_{\mathrm{F}}^{2}=\sum_{i, j=1}^{n} \frac{\left|\tilde{\lambda}_{i}-\lambda_{j}\right|}{\sqrt{\tilde{\lambda}_{i} \lambda_{j}}}\left|q_{i j}\right|^{2} .
$$

Since $\left(\left|q_{i j}\right|^{2}\right)$ is a doubly stochastic matrix, applying Lemma 8.2 and Proposition 3.13 concludes the proof of inequality (5.2). To confirm (5.1), let $k$ be the index such that

$$
\eta_{p} \stackrel{\text { def }}{=} \max _{1 \leq i \leq n} \chi\left(\lambda_{i}, \tilde{\lambda}_{i}\right)=\chi\left(\lambda_{k}, \tilde{\lambda}_{k}\right)
$$

If $\eta_{p}=0$, no proof is necessary. Assume $\eta_{p}>0$. Also assume, without loss of any generality, that

$$
\lambda_{k}>\widetilde{\lambda}_{k} \geq 0
$$

Partition $U, V, \tilde{U}, \tilde{V}$ as follows

$$
U=\left(U_{1}, U_{2}\right), V=\left(V_{1}, V_{2}\right), \tilde{U}=\left(\tilde{U}_{1}, \tilde{U}_{2}\right) \text { and } \tilde{V}=\left(\tilde{V}_{1}, \tilde{V}_{2}\right),
$$

where $U_{1}, V_{1} \in \mathbf{C}^{n \times k}$ and $\tilde{U}_{1}, \tilde{V}_{1} \in \mathbf{C}^{n \times(k-1)}$, and write $\Lambda=\operatorname{diag}\left(\Lambda_{1}, \Lambda_{2}\right)$ and $\tilde{\Lambda}=$ $\operatorname{diag}\left(\tilde{\Lambda}_{1}, \widetilde{\Lambda}_{2}\right)$, where $\Lambda_{1} \in \mathbf{R}^{k \times k}$ and $\widetilde{\Lambda}_{1} \in \mathbf{R}^{(k-1) \times(k-1)}$. It follows from equation (9.1) that

$$
\tilde{\Lambda}_{2} \tilde{U}_{2}^{*} U_{1}-\tilde{U}_{2}^{*} U_{1} \Lambda_{1}=\tilde{\Lambda}_{2}^{1 / 2} \tilde{V}_{2}^{*}\left(D^{*}-D^{-1}\right) V_{1} \Lambda_{1}^{1 / 2}
$$


Post-multiply this equation by $\Lambda_{1}^{-1}$ to get

$$
\widetilde{\Lambda}_{2} \tilde{U}_{2}^{*} U_{1} \Lambda_{1}^{-1}-\tilde{U}_{2}^{*} U_{1}=\tilde{\Lambda}_{2}^{1 / 2} \tilde{V}_{2}^{*}\left(D^{*}-D^{-1}\right) V_{1} \Lambda_{1}^{-1 / 2}
$$

Lemma 8.4 implies that $\left\|\widetilde{U}_{2}^{*} U_{1}\right\|_{2}=1$ since $\tilde{U}_{2}^{*} U_{1}$ is a $(n-k+1) \times k$ submatrix of $\tilde{U}^{*} U$ and $k+(n-k+1)=n+1>n$. Bearing in mind that $\left\|\tilde{\Lambda}_{2}\right\|_{2}=\tilde{\lambda}_{k}=\left\|\tilde{\Lambda}_{2}^{1 / 2}\right\|_{2}^{2}$ and $\left\|\Lambda_{1}^{-1}\right\|_{2}=1 / \lambda_{k}=\left\|\Lambda_{1}^{-1 / 2}\right\|_{2}^{2}$, we have

$$
\begin{aligned}
1-\frac{\tilde{\lambda}_{k}}{\lambda_{k}} & =\left\|\tilde{U}_{2}^{*} U_{1}\right\|_{2}-\left\|\tilde{\Lambda}_{2}\right\|_{2}\left\|\tilde{U}_{2}^{*} U_{1}\right\|_{2}\left\|\Lambda_{1}^{-1}\right\|_{2} \\
& \leq\left\|\tilde{U}_{2}^{*} U_{1}\right\|_{2}-\left\|\tilde{\Lambda}_{2} \tilde{U}_{2}^{*} U_{1} \Lambda_{1}^{-1}\right\|_{2} \\
& \leq\left\|\tilde{U}_{2}^{*} U_{1}-\tilde{\Lambda}_{2} \tilde{U}_{2}^{*} U_{1} \Lambda_{1}^{-1}\right\|_{2} \\
& =\left\|\tilde{\Lambda}_{2}^{1 / 2} \tilde{V}_{2}^{*}\left(D^{*}-D^{-1}\right) V_{1} \Lambda_{1}^{-1 / 2}\right\|_{2} \\
& \leq\left\|\tilde{\Lambda}_{2}^{1 / 2}\right\|_{2}\left\|\tilde{V}_{2}^{*}\left(D^{*}-D^{-1}\right) V_{1}\right\|_{2}\left\|\Lambda_{1}^{-1 / 2}\right\|_{2} \\
& =\sqrt{\frac{\tilde{\lambda}_{k}}{\lambda_{k}}}\left\|\tilde{V}_{2}^{*}\left(D^{*}-D^{-1}\right) V_{1}\right\|_{2} \\
& \leq \sqrt{\frac{\tilde{\lambda}_{k}}{\lambda_{k}}}\left\|D^{*}-D^{-1}\right\|_{2},
\end{aligned}
$$

an immediate consequence of which is inequality (5.1).

Lemma 9.1 For $\alpha, \beta, \gamma>0$, we have

$$
\chi(\alpha, \gamma) \leq \chi(\alpha, \beta)+\chi(\beta, \gamma)+\frac{1}{8} \chi(\alpha, \beta) \chi(\beta, \gamma) \chi(\alpha, \gamma)
$$

Thus if $\chi(\alpha, \beta) \chi(\beta, \gamma)<8$, then

$$
\chi(\alpha, \gamma) \leq \frac{\chi(\alpha, \beta)+\chi(\beta, \gamma)}{1-\frac{1}{8} \chi(\alpha, \beta) \chi(\beta, \gamma)}
$$

Proof: Without loss of any generality, we may assume $\alpha \leq \gamma$. Now if $\beta \leq \alpha$ or $\gamma \leq \beta$, by Property 7 of Proposition 3.7

$$
\chi(\alpha, \gamma) \leq \begin{cases}\chi(\beta, \gamma) \leq \chi(\alpha, \beta)+\chi(\beta, \gamma), & \text { if } \beta \leq \alpha \\ \chi(\alpha, \beta) \leq \chi(\alpha, \beta)+\chi(\beta, \gamma), & \text { if } \gamma \leq \beta\end{cases}
$$

So inequality (9.3) has to be true. Consider the case $\alpha \leq \beta \leq \gamma$. From the proof of Proposition 3.14, we have

$$
\chi(\alpha, \gamma)=\chi(\alpha, \beta)+\chi(\beta, \gamma)+\chi(\sqrt{\alpha}, \sqrt{\beta}) \chi(\sqrt{\beta}, \sqrt{\gamma}) \chi(\sqrt{\alpha}, \sqrt{\gamma}) .
$$


By inequality (3.19) of Proposition 3.10, we get immediately inequality (9.3).

Proof of Theorems 5.2 and 5.3: Set $\widehat{B}=B D_{2}$ and denote its singular values by $\widehat{\sigma}_{1} \geq \widehat{\sigma}_{2} \geq$ $\cdots \geq \widehat{\sigma}_{n}$. Applying Theorem 5.1 to $B^{*} B$ and $\widehat{B}^{*} \widehat{B}=D_{2}^{*} B^{\star} B D_{2}$ leads to

$$
\max _{1 \leq i \leq n} \chi\left(\sigma_{i}^{2}, \hat{\sigma}_{i}^{2}\right) \leq\left\|D_{2}^{*}-D_{2}^{-1}\right\|_{2} \text { and } \sqrt{\sum_{i=1}^{n}\left[\chi\left(\sigma_{i}^{2}, \hat{\sigma}_{i}^{2}\right)\right]^{2}} \leq\left\|D_{2}^{*}-D_{2}^{-1}\right\|_{\mathrm{F}} \text {. }
$$

Now apply inequality (3.19) of Proposition 3.10 to obtain

$$
\max _{1 \leq i \leq n} \chi\left(\sigma_{i}, \widehat{\sigma}_{i}\right) \leq \frac{1}{2}\left\|D_{2}^{*}-D_{2}^{-1}\right\|_{2} \quad \text { and } \quad \sqrt{\sum_{i=1}^{n}\left[\chi\left(\sigma_{i}, \widehat{\sigma}_{i}\right)\right]^{2}} \leq \frac{1}{2}\left\|D_{2}^{*}-D_{2}^{-1}\right\|_{\mathrm{F}} .
$$

Similarly for $\widehat{B}=B D_{2}$ and $\widetilde{B}=D_{1}^{*} B D_{2}=D_{1}^{*} \widehat{B}$, we have

$$
\max _{1 \leq i \leq n} \chi\left(\hat{\sigma}_{i}, \tilde{\sigma}_{i}\right) \leq \frac{1}{2}\left\|D_{1}^{*}-D_{1}^{-1}\right\|_{2} \quad \text { and } \quad \sqrt{\sum_{i=1}^{n}\left[\chi\left(\hat{\sigma}_{i}, \tilde{\sigma}_{i}\right)\right]^{2}} \leq \frac{1}{2}\left\|D_{1}^{*}-D_{1}^{-1}\right\|_{\mathrm{F}} .
$$

Under the assumptions of Theorem 5.2, by Lemma 9.1, we have

$$
\chi\left(\sigma_{i}, \widehat{\sigma}_{i}\right) \chi\left(\widehat{\sigma}_{i}, \tilde{\sigma}_{i}\right) \leq \frac{1}{4}\left\|D_{1}^{*}-D_{1}^{-1}\right\|_{2}\left\|D_{2}^{*}-D_{2}^{-1}\right\|_{2}<\frac{1}{4} \times 32=8 ;
$$

so we have

$$
\begin{aligned}
\chi\left(\sigma_{i}, \tilde{\sigma}_{i}\right) & \leq \frac{\chi\left(\sigma_{i}, \hat{\sigma}_{i}\right)+\chi\left(\hat{\sigma}_{i}, \tilde{\sigma}_{i}\right)}{1-\frac{1}{8} \chi\left(\sigma_{i}, \hat{\sigma}_{i}\right) \chi\left(\hat{\sigma}_{i}, \tilde{\sigma}_{i}\right)} \\
& \leq \frac{1}{2} \cdot \frac{\left\|D_{1}^{*}-D_{1}^{-1}\right\|_{2}+\left\|D_{2}^{*}-D_{2}^{-1}\right\|_{2}}{1-\frac{1}{32}\left\|D_{1}^{*}-D_{1}^{-1}\right\|_{2}\left\|D_{2}^{*}-D_{2}^{-1}\right\|_{2}} \\
\sqrt{\sum_{i=1}^{n}\left[\chi\left(\sigma_{i}, \tilde{\sigma}_{i}\right)\right]^{2}} & \leq \sqrt{\sum_{i=1}^{n}\left[\frac{\chi\left(\sigma_{i}, \hat{\sigma}_{i}\right)+\chi\left(\hat{\sigma}_{i}, \tilde{\sigma}_{i}\right)}{1-\frac{1}{8} \chi\left(\sigma_{i}, \hat{\sigma}_{i}\right) \chi\left(\widehat{\sigma}_{i}, \tilde{\sigma}_{i}\right)}\right]^{2}} \\
& \leq \frac{\sqrt{\sum_{i=1}^{n}\left[\chi\left(\sigma_{i}, \hat{\sigma}_{i}\right)\right]^{2}}+\sqrt{\sum_{i=1}^{n}\left[\chi\left(\hat{\sigma}_{i}, \tilde{\sigma}_{i}\right)\right]^{2}}}{1-\frac{1}{8} \max _{1 \leq i \leq n} \chi\left(\sigma_{i}, \hat{\sigma}_{i}\right) \chi\left(\hat{\sigma}_{i}, \tilde{\sigma}_{i}\right)} \\
& \leq \frac{1}{2} \cdot \frac{\left\|D_{1}^{*}-D_{1}^{-1}\right\|_{\mathrm{F}}+\left\|D_{2}^{*}-D_{2}^{-1}\right\|_{\mathrm{F}}}{1-\frac{1}{32}\left\|D_{1}^{*}-D_{1}^{-1}\right\|_{2}\left\|D_{2}^{*}-D_{2}^{-1}\right\|_{2}}
\end{aligned}
$$

as expected. This completes the proof of Theorem 5.2. To prove Theorem 5.3, we notice that

$$
\begin{aligned}
\varrho_{p}\left(\sigma_{i}, \widetilde{\sigma}_{i}\right) & \leq \varrho_{p}\left(\sigma_{i}, \hat{\sigma}_{i}\right)+\varrho_{p}\left(\hat{\sigma}_{i}, \tilde{\sigma}_{i}\right) & & \left(\varrho_{p} \text { is a metric on } \mathbf{R}\right) \\
& \leq 2^{-1 / p} \chi\left(\sigma_{i}, \hat{\sigma}_{i}\right)+2^{-1 / p} \chi\left(\hat{\sigma}_{i}, \tilde{\sigma}_{i}\right) & & (\text { by Proposition } 3.11) \\
& \leq 2^{-1-1 / p}\left(\left\|D_{2}^{*}-D_{2}^{-1}\right\|_{2}+\left\|D_{1}^{*}-D_{1}^{-1}\right\|_{2}\right), & & (\text { by }(9.4) \text { and }(9.5))
\end{aligned}
$$


and

$$
\begin{aligned}
& \sqrt{\sum_{i=1}^{n}\left[\varrho_{p}\left(\sigma_{i}, \tilde{\sigma}_{i}\right)\right]^{2}} \leq \sqrt{\sum_{i=1}^{n}\left[\varrho_{p}\left(\sigma_{i}, \hat{\sigma}_{i}\right)+\varrho_{p}\left(\hat{\sigma}_{i}, \tilde{\sigma}_{i}\right)\right]^{2}} \quad\left(\varrho_{p} \text { is a metric on } \mathbf{R}\right) \\
& \leq \sqrt{\sum_{i=1}^{n}\left[\varrho_{p}\left(\sigma_{i}, \hat{\sigma}_{i}\right)\right]^{2}}+\sqrt{\sum_{i=1}^{n}\left[\varrho_{p}\left(\hat{\sigma}_{i}, \tilde{\sigma}_{i}\right)\right]^{2}} \\
& \leq 2^{-1 / p} \sqrt{\sum_{i=1}^{n}\left[\chi\left(\sigma_{i}, \hat{\sigma}_{i}\right)\right]^{2}}+2^{-1 / p} \sqrt{\sum_{i=1}^{n}\left[\chi\left(\hat{\sigma}_{i}, \tilde{\sigma}_{i}\right)\right]^{2}} \\
& \text { (by Proposition 3.11) } \\
& \leq 2^{-1-1 / p}\left(\left\|D_{2}^{*}-D_{2}^{-1}\right\|_{\mathrm{F}}+\left\|D_{1}^{*}-D_{1}^{-1}\right\|_{\mathrm{F}}\right) \text {. (by (9.4) and (9.5)) }
\end{aligned}
$$

These inequalities complete the proof of Theorem 5.3.

Proof of Theorem 5.4: Rewrite $A$ and $\tilde{A}$ as

$$
\begin{aligned}
A & =S^{*} H S=\left(H^{1 / 2} S\right)^{*} H^{1 / 2} S \\
\widetilde{A} & =S^{*} H^{1 / 2}\left(I+H^{-1 / 2}(\Delta H) H^{-1 / 2}\right) H^{1 / 2} S \\
& =\left(\left(I+H^{-1 / 2}(\Delta H) H^{-1 / 2}\right)^{1 / 2} H^{1 / 2} S\right)^{*}\left(I+H^{-1 / 2}(\Delta H) H^{-1 / 2}\right)^{1 / 2} H^{1 / 2} S .
\end{aligned}
$$

Set $B \stackrel{\text { def }}{=} H^{1 / 2} S$ and $\widetilde{B} \stackrel{\text { def }}{=}\left(I+H^{-1 / 2}(\Delta H) H^{-1 / 2}\right)^{1 / 2} H^{1 / 2} S$, then $A=B^{*} B$ and $\widetilde{A}=\widetilde{B}^{*} \widetilde{B}$. Set $D=\left(I+H^{-1 / 2}(\Delta H) H^{-1 / 2}\right)^{1 / 2}$, then $\widetilde{B}=D B$. Notice that $\lambda(A)=\lambda\left(B^{*} B\right)=\lambda\left(B B^{*}\right)$ and $\lambda(\widetilde{A})=\lambda\left(\widetilde{B}^{*} \widetilde{B}\right)=\lambda\left(\widetilde{B} \widetilde{B}^{*}\right)$ and $\widetilde{B} \widetilde{B}^{*}=D B B^{*} D^{*}$. Applying Theorem 5.1 to $B B^{*}$ and $\widetilde{B} \widetilde{B}^{*}$ yields the first " $\leq$ " in both (5.11) and (5.12).

Proof of Theorem 5.5: Write

$$
\widetilde{B}=(G+\Delta G) S=\left(I+(\Delta G) G^{-1}\right) G S=D B,
$$

where $D=I+(\Delta G) G^{-1}$. Now applying Theorem 5.2 to $B$ and $\widetilde{B}=D B$ yields the first inequalities in both (5.13) and (5.14). To get the second inequalities, we notice

$$
(I+E)^{*}-(I+E)^{-1}=I+E^{*}-\sum_{i=0}^{\infty}(-1)^{i} E^{i}=E^{*}+E+E \sum_{i=2}^{\infty}(-1)^{i} E^{i-1},
$$

where $E=(\Delta G) G^{-1}$ and $\|E\|_{2} \leq\left\|G^{-1}\right\|_{2}\|\Delta G\|_{2}<1$; therefore for any unitarily invariant norm $\|\cdot\|$

$$
\begin{aligned}
\left\|(I+E)^{*}-(I+E)^{-1}\right\| & \leq\left\|E+E^{*}\right\|+\|E\| \sum_{i=1}^{\infty}\|E\|_{2}^{i} \\
& =\left(\frac{\left\|E+E^{*}\right\|}{\|E\|}+\frac{\|E\|_{2}}{1-\|E\|_{2}}\right)\|E\| .
\end{aligned}
$$

The rest is just applications of this inequality for $\|\cdot\|\|=\| \cdot \|_{2}$ and for $\|\cdot\|=\|\cdot\|_{\mathrm{F}}$. 


\section{Proof of Theorem 6.6}

No proof is necessary if $\tilde{\lambda} \in \lambda(A)$. Assume that $\tilde{\lambda} \notin \lambda(A)$. Here we consider the case when $\widetilde{A}=D A$ only, since the situation for the case when $\widetilde{A}=A D$ is very similar.

$$
\begin{aligned}
\tilde{A}-\tilde{\lambda} I & =A-\tilde{\lambda} I+\tilde{A}-A \\
& =X(\Lambda-\tilde{\lambda} I) X^{-1}+(D-I) X \Lambda X^{-1} \\
& =X\left[I+X^{-1}(D-I) X \Lambda(\Lambda-\tilde{\lambda} I)^{-1}\right](\Lambda-\tilde{\lambda} I) X^{-1}
\end{aligned}
$$

Since $\tilde{A}-\tilde{\lambda} I$ is singular, we have for any $1 \leq p \leq \infty,\left\|X^{-1}(D-I) X \Lambda(\Lambda-\tilde{\lambda} I)^{-1}\right\|_{p} \geq 1$ which gives

$$
1 \leq\left\|X^{-1}(D-I) X\right\|_{p}\left\|\Lambda(\Lambda-\tilde{\lambda} I)^{-1}\right\|_{p}=\left\|X^{-1}(D-I) X\right\|_{p} \max _{\lambda \in \lambda(A)} \frac{|\lambda|}{|\widetilde{\lambda}-\lambda|}
$$

as was to be shown.

\section{Generalized Eigenvalue Problems and Generalized Sin- gular Value Problems}

In this section, we are going to say a few words for the following perturbations for Scaled Generalized Eigenvalue Problems and Scaled Generalized Singular Value Problems. As we shall see, the results in previous sections, as well as those in $\mathrm{Li}$ [24, 1994], can be applied to derive relative perturbation bounds for them.

- Generalized eigenvalue problem:

$A_{1}-\lambda A_{2} \equiv S_{1}^{*} H_{1} S_{1}-\lambda S_{2}^{*} H_{2} S_{2}$ and $\widetilde{A}_{1}-\lambda \widetilde{A}_{2} \equiv S_{1}^{*} \widetilde{H}_{1} S_{1}-\lambda S_{2}^{*} \widetilde{H}_{2} S_{2}$, where $H_{1}$ and $H_{2}$ are positive definite and $\left\|H_{i}^{-1}\right\|_{2}\left\|\widetilde{H}_{i}-H_{i}\right\|_{2}<1$ for $i=1,2$, and $S_{1}$ and $S_{2}$ are some square matrices and one of them is nonsingular.

- Generalized singular problem:

$\left\{B_{1}, B_{2}\right\} \equiv\left\{G_{1} S_{1}, G_{2} S_{2}\right\}$ and $\left\{\widetilde{B}_{1}, \widetilde{B}_{2}\right\} \equiv\left\{\widetilde{G}_{1} S_{1}, \widetilde{G}_{2} S_{2}\right\}$, where $G_{1}$ and $G_{2}$ are nonsingular and $\left\|G_{i}^{-1}\right\|_{2}\left\|\widetilde{G}_{i}-G_{i}\right\|_{2}<1$ for $i=1,2$, and $S_{1}$ and $S_{2}$ are some square matrices and one of them is nonsingular.

For the scaled generalized eigenvalue problem just mentioned, without loss of any generality, we consider the case when $S_{2}$ is nonsingular. Then the generalized eigenvalue problem for $A_{1}-\lambda A_{2} \equiv S_{1}^{*} H_{1} S_{1}-\lambda S_{2}^{*} H_{2} S_{2}$ is equivalent to the standard eigenvalue problem for

$$
A \stackrel{\text { def }}{=} H_{2}^{-1 / 2} S_{2}^{-1} S_{1}^{*} H_{1} S_{1} S_{2}^{-1} H_{2}^{-1 / 2} \text {; }
$$

and the generalized eigenvalue problem for $\widetilde{A}_{1}-\lambda \widetilde{A}_{2} \equiv S_{1}^{*} \widetilde{H}_{1} S_{1}-\lambda S_{2}^{*} \widetilde{H}_{2} S_{2}$ is equivalent to the standard eigenvalue problem for

$$
\widetilde{A} \stackrel{\text { def }}{=} D_{2}^{*} H_{2}^{-1 / 2} S_{2}^{-1} S_{1}^{*} \widetilde{H}_{1} S_{1} S_{2}^{-1} H_{2}^{-1 / 2} D_{2},
$$


where

$$
D_{2}=D_{2}^{*} \stackrel{\text { def }}{=}\left(I+H_{2}^{-1 / 2}\left(\Delta H_{2}\right) H_{2}^{-1 / 2}\right)^{-1 / 2} \text { and } \quad \Delta H_{2} \stackrel{\text { def }}{=} \widetilde{H}_{2}-H_{2} .
$$

So bounding relative distances between the eigenvalues of $A_{1}-\lambda A_{2}$ and these of $\widetilde{A}_{1}-\lambda \widetilde{A}_{2}$ is transformed to bounding relative distances between the eigenvalues of the matrix $A$ and these of the matrix $\tilde{A}$. The latter can be accomplished in two steps:

1. Bounding relative distances between the eigenvalues of the matrix $A$ and these of

$$
\widehat{A} \stackrel{\text { def }}{=} D_{2}^{*} H_{2}^{-1 / 2} S_{2}^{-1} S_{1}^{*} H_{1} S_{1} S_{2}^{-1} H_{2}^{-1 / 2} D_{2} .
$$

2. Bounding relative distances between the eigenvalues of the matrix $\hat{A}$ and these of the matrix $\tilde{A}$.

Denote and order the eigenvalues of $A, \widehat{A}$ and $\tilde{A}$ as

$$
\lambda_{1} \geq \cdots \geq \lambda_{n} \text { and } \hat{\lambda}_{1} \geq \cdots \geq \hat{\lambda}_{n} \quad \text { and } \quad \tilde{\lambda}_{1} \geq \cdots \geq \tilde{\lambda}_{n}
$$

Set

$$
D_{1}=D_{1}^{*} \stackrel{\text { def }}{=}\left(I+H_{1}^{-1 / 2}\left(\Delta H_{1}\right) H_{1}^{-1 / 2}\right)^{-1 / 2} \quad \text { and } \quad \Delta H_{1} \stackrel{\text { def }}{=} \widetilde{H}_{1}-H_{1} .
$$

By Theorems 5.1 and 5.4 , we have for $1 \leq j \leq n$

$$
\chi\left(\lambda_{i}, \hat{\lambda}_{i}\right) \leq\left\|D_{2}-D_{2}^{-1}\right\|_{2} \quad \text { and } \quad \chi\left(\hat{\lambda}_{i}, \tilde{\lambda}_{i}\right) \leq\left\|D_{1}-D_{1}^{-1}\right\|_{2}
$$

and

$$
\sqrt{\sum_{i=1}^{n}\left[\chi\left(\lambda_{i}, \hat{\lambda}_{i}\right)\right]^{2}} \leq\left\|D_{2}-D_{2}^{-1}\right\|_{\mathrm{F}} \quad \text { and } \quad \sqrt{\sum_{i=1}^{n}\left[\chi\left(\hat{\lambda}_{i}, \tilde{\lambda}_{i}\right)\right]^{2}} \leq\left\|D_{1}-D_{1}^{-1}\right\|_{\mathrm{F}} \text {. }
$$

By Lemma 9.1 , we have for $1 \leq j \leq n$ if $\left\|D_{1}-D_{1}^{-1}\right\|_{2}\left\|D_{2}-D_{2}^{-1}\right\|_{2}<8$,

$$
\chi\left(\lambda_{i}, \tilde{\lambda}_{i}\right) \leq \frac{\chi\left(\lambda_{i}, \hat{\lambda}_{i}\right)+\chi\left(\hat{\lambda}_{i}, \tilde{\lambda}_{i}\right)}{1-\frac{1}{8} \chi\left(\lambda_{i}, \hat{\lambda}_{i}\right) \chi\left(\hat{\lambda}_{i}, \widetilde{\lambda}_{i}\right)} \leq \frac{\left\|D_{2}-D_{2}^{-1}\right\|_{2}+\left\|D_{1}-D_{1}^{-1}\right\|_{2}}{1-\frac{1}{8}\left\|D_{1}-D_{1}^{-1}\right\|_{2}\left\|D_{2}-D_{2}^{-1}\right\|_{2}}
$$

and

$$
\begin{aligned}
\sqrt{\sum_{i=1}^{n}\left[\chi\left(\lambda_{i}, \tilde{\lambda}_{i}\right)\right]^{2}} & \leq \sqrt{\sum_{i=1}^{n}\left[\frac{\chi\left(\lambda_{i}, \hat{\lambda}_{i}\right)+\chi\left(\hat{\lambda}_{i}, \tilde{\lambda}_{i}\right)}{1-\frac{1}{8} \chi\left(\lambda_{i}, \hat{\lambda}_{i}\right) \chi\left(\hat{\lambda}_{i}, \tilde{\lambda}_{i}\right)}\right]^{2}} \\
& \leq \frac{\sqrt{\sum_{i=1}^{n}\left[\chi\left(\lambda_{i}, \hat{\lambda}_{i}\right)\right]^{2}}+\sqrt{\sum_{i=1}^{n}\left[\chi\left(\hat{\lambda}_{i}, \tilde{\lambda}_{i}\right)\right]^{2}}}{1-\frac{1}{8} \max _{1 \leq i \leq n} \chi\left(\lambda_{i}, \hat{\lambda}_{i}\right) \chi\left(\hat{\lambda}_{i}, \tilde{\lambda}_{i}\right)} \\
& \leq \frac{\left\|D_{2}-D_{2}^{-1}\right\|_{\mathrm{F}}+\left\|D_{1}-D_{1}^{-1}\right\|_{\mathrm{F}}}{1-\frac{1}{8}\left\|D_{1}-D_{1}^{-1}\right\|_{2}\left\|D_{2}-D_{2}^{-1}\right\|_{2}}
\end{aligned}
$$


Notice also that for $i=1,2$ and for any unitarily invariant norm $\|\cdot\|$

$$
\left\|D_{i}-D_{i}^{-1}\right\| \leq \frac{\left\|H_{i}^{-1}\right\|_{2}\left\|\Delta H_{i}\right\|}{\sqrt{1-\left\|H_{i}^{-1}\right\|_{2}\left\|\Delta H_{i}\right\|_{2}}} .
$$

So we have proved

Theorem 11.1 Let $A_{1}-\lambda A_{2} \equiv S_{1}^{*} H_{1} S_{1}-\lambda S_{2}^{*} H_{2} S_{2}$ and $\tilde{A}_{1}-\lambda \widetilde{A}_{2} \equiv S_{1}^{*} \tilde{H}_{1} S_{1}-\lambda S_{2}^{*} \tilde{H}_{2} S_{2}$, where $H_{1}$ and $H_{2}$ are $n \times n$ and positive definite and $\left\|H_{i}^{-1}\right\|_{2}\left\|\widetilde{H}_{i}-H_{i}\right\|_{2}<1$ for $i=1,2$, and $S_{1}$ and $S_{2}$ are some square matrices and one of them is nonsingular. Let the generalized eigenvalues of $A_{1}-\lambda A_{2}$ and $\tilde{A}_{1}-\lambda \tilde{A}_{2}$ be

$$
\lambda_{1} \geq \cdots \geq \lambda_{n} \text { and } \tilde{\lambda}_{1} \geq \cdots \geq \tilde{\lambda}_{n}
$$

If $\left\|D_{1}-D_{1}^{-1}\right\|_{2}\left\|D_{2}-D_{2}^{-1}\right\|_{2}<8$, then

$$
\begin{aligned}
\max _{1 \leq i \leq n} \chi\left(\lambda_{i}, \tilde{\lambda}_{i}\right) & \leq \frac{\theta_{1}\left\|\Delta H_{1}\right\|_{2}+\theta_{2}\left\|\Delta H_{2}\right\|_{2}}{1-\frac{1}{8} \theta_{1} \theta_{2}\left\|\Delta H_{1}\right\|_{2}\left\|\Delta H_{2}\right\|_{2}}, \\
\sqrt{\sum_{i=1}^{n}\left[\chi\left(\lambda_{i}, \tilde{\lambda}_{i}\right)\right]^{2}} & \leq \frac{\theta_{1}\left\|\Delta H_{1}\right\|_{\mathrm{F}}+\theta_{2}\left\|\Delta H_{2}\right\|_{\mathrm{F}}}{1-\frac{1}{8} \theta_{1} \theta_{2}\left\|\Delta H_{1}\right\|_{2}\left\|\Delta H_{2}\right\|_{2}},
\end{aligned}
$$

where $\theta_{i} \stackrel{\text { def }}{=}\left\|H_{i}^{-1}\right\|_{2} / \sqrt{1-\left\|H_{i}^{-1}\right\|_{2}\left\|\Delta H_{i}\right\|_{2}}$ for $i=1,2$.

On the other hand, from (11.1) and (11.2) and Proposition 3.11, we get

$$
\varrho_{p}\left(\lambda_{i}, \hat{\lambda}_{i}\right) \leq 2^{-1 / p}\left\|D_{2}-D_{2}^{-1}\right\|_{2} \quad \text { and } \quad \varrho_{p}\left(\hat{\lambda}_{i}, \widetilde{\lambda}_{i}\right) \leq 2^{-1 / p}\left\|D_{1}-D_{1}^{-1}\right\|_{2}
$$

and

$$
\sqrt{\sum_{i=1}^{n}\left[\varrho_{p}\left(\lambda_{i}, \hat{\lambda}_{i}\right)\right]^{2}} \leq 2^{-1 / p}\left\|D_{2}-D_{2}^{-1}\right\|_{\mathrm{F}} \quad \text { and } \quad \sqrt{\sum_{i=1}^{n}\left[\varrho_{p}\left(\widehat{\lambda}_{i}, \tilde{\lambda}_{i}\right)\right]^{2}} \leq 2^{-1 / p}\left\|D_{1}-D_{1}^{-1}\right\|_{\mathrm{F}} .
$$

Since $\varrho_{p}$ is a metric on $\mathbf{R}$, we have for $1 \leq j \leq n$

$$
\varrho_{p}\left(\lambda_{i}, \tilde{\lambda}_{i}\right) \leq \varrho_{p}\left(\lambda_{i}, \hat{\lambda}_{i}\right)+\varrho_{p}\left(\hat{\lambda}_{i}, \tilde{\lambda}_{i}\right) \leq 2^{-1 / p}\left(\left\|D_{2}-D_{2}^{-1}\right\|_{2}+\left\|D_{1}-D_{1}^{-1}\right\|_{2}\right)
$$

and

$$
\begin{aligned}
\sqrt{\sum_{i=1}^{n}\left[\varrho_{p}\left(\lambda_{i}, \tilde{\lambda}_{i}\right)\right]^{2}} & \leq \sqrt{\sum_{i=1}^{n}\left[\varrho_{p}\left(\lambda_{i}, \hat{\lambda}_{i}\right)+\varrho_{p}\left(\hat{\lambda}_{i}, \tilde{\lambda}_{i}\right)\right]^{2}} \\
& \leq \sqrt{\sum_{i=1}^{n}\left[\varrho_{p}\left(\lambda_{i}, \hat{\lambda}_{i}\right)\right]^{2}}+\sqrt{\sum_{i=1}^{n}\left[\varrho_{p}\left(\hat{\lambda}_{i}, \tilde{\lambda}_{i}\right)\right]^{2}} \\
& \leq 2^{-1 / p}\left(\left\|D_{2}-D_{2}^{-1}\right\|_{\mathrm{F}}+\left\|D_{1}-D_{1}^{-1}\right\|_{\mathrm{F}}\right)
\end{aligned}
$$


Theorem 11.2 Let all conditions of Theorem 11.1, except $\left\|D_{1}-D_{1}^{-1}\right\|_{2}\left\|D_{2}-D_{2}^{-1}\right\|_{2}<8$, hold. Then

$$
\begin{aligned}
\max _{1 \leq i \leq n} \varrho_{p}\left(\lambda_{i}, \tilde{\lambda}_{i}\right) & \leq 2^{-1 / p}\left(\theta_{1}\left\|\Delta H_{1}\right\|_{2}+\theta_{2}\left\|\Delta H_{2}\right\|_{2}\right), \\
\sqrt{\sum_{i=1}^{n}\left[\varrho_{p}\left(\lambda_{i}, \tilde{\lambda}_{i}\right)\right]^{2}} & \leq 2^{-1 / p}\left(\theta_{1}\left\|\Delta H_{1}\right\|_{\mathrm{F}}+\theta_{2}\left\|\Delta H_{2}\right\|_{\mathrm{F}}\right) .
\end{aligned}
$$

As to the scaled generalized singular problem mentioned above, we shall consider its corresponding generalized eigenvalue problem [22, 37, 39] for

$$
S_{1}^{*} G_{1}^{*} G_{1} S_{1}-\lambda S_{2}^{*} G_{2}^{*} G_{2} S_{2} \text { and } S_{1}^{*} \widetilde{G}_{1}^{*} \widetilde{G}_{1} S_{1}-\lambda S_{2}^{*} \widetilde{G}_{2}^{*} \widetilde{G}_{2} S_{2},
$$

instead.

Theorem 11.3 Let $\left\{B_{1}, B_{2}\right\} \equiv\left\{G_{1} S_{1}, G_{2} S_{2}\right\}$ and $\left\{\widetilde{B}_{1}, \widetilde{B}_{2}\right\} \equiv\left\{\widetilde{G}_{1} S_{1}, \widetilde{G}_{2} S_{2}\right\}$, where $G_{1}$ and $G_{2}$ are $n \times n$ and nonsingular and $\left\|G_{i}^{-1}\right\|_{2}\left\|\widetilde{G}_{i}-G_{i}\right\|_{2}<1$ for $i=1,2$, and $S_{1}$ and $S_{2}$ are some square matrices and one of them is nonsingular. Let the generalized singular values of $\left\{B_{1}, B_{2}\right\}$ and $\left\{\widetilde{B}_{1}, \widetilde{B}_{2}\right\}$ be

$$
\sigma_{1} \geq \cdots \geq \sigma_{n} \quad \text { and } \quad \tilde{\sigma}_{1} \geq \cdots \geq \tilde{\sigma}_{n} .
$$

If $\delta_{12} \delta_{22}<32$, where

$$
\delta_{i t}=\left\|\left(I+\left(\Delta G_{i}\right) G_{i}^{-1}\right)^{*}-\left(I+\left(\Delta G_{i}\right) G_{i}^{-1}\right)^{-1}\right\|_{t} \quad \text { for } i=1,2 \text { and } t=2, \mathrm{~F},
$$

then

$$
\begin{aligned}
\max _{1 \leq i \leq n} \chi\left(\sigma_{i}, \tilde{\sigma}_{i}\right) & \leq \frac{1}{2} \cdot \frac{\delta_{12}+\delta_{22}}{1-\frac{1}{32} \delta_{12} \delta_{22}} \\
\sqrt{\sum_{i=1}^{n}\left[\chi\left(\sigma_{i}, \tilde{\sigma}_{i}\right)\right]^{2}} & \leq \frac{1}{2} \cdot \frac{\delta_{1 \mathrm{~F}}+\delta_{2 \mathrm{~F}}}{1-\frac{1}{32} \delta_{12} \delta_{22}} .
\end{aligned}
$$

It can be proved that for $i=1,2$ and $t=2, \mathrm{~F}$

$$
\begin{aligned}
\delta_{i t} & \leq\left(\frac{\left\|\left(\Delta G_{i}\right) G_{i}^{-1}+G_{i}^{-*}\left(\Delta G_{i}\right)^{*}\right\|_{t}}{\left\|\left(\Delta G_{i}\right) G_{i}^{-1}\right\|_{t}}+\frac{\left\|\left(\Delta G_{i}\right) G_{i}^{-1}\right\|_{2}}{1-\left\|\left(\Delta G_{i}\right) G_{i}^{-1}\right\|_{2}}\right)\left\|\left(\Delta G_{i}\right) G_{i}^{-1}\right\|_{t} \\
& \leq\left(1+\frac{1}{1-\left\|G_{i}^{-1}\right\|_{2}\left\|\Delta G_{i}\right\|_{2}}\right)\left\|G_{i}^{-1}\right\|_{2}\left\|\Delta G_{i}\right\|_{t} .
\end{aligned}
$$

Proof: Consider the case when $S_{2}$ is nonsingular. (The case when $S_{1}$ is nonsingular can be handled analogously.) By (11.3), we know that the singular values of $B \stackrel{\text { def }}{=} G_{1} S_{1} S_{2}^{-1} G_{2}^{-1}$ and $\widetilde{B} \stackrel{\text { def }}{=} \widetilde{G}_{1} S_{1} S_{2}^{-1} \widetilde{G}_{2}^{-1}$ are $\sigma_{1} \geq \cdots \geq \sigma_{n}$ and $\widetilde{\sigma}_{1} \geq \cdots \geq \widetilde{\sigma}_{n}$. Set

$D_{1}=I+\left(\Delta G_{1}\right) G_{1}^{-1}, \quad \Delta G_{1}=\widetilde{G}_{1}-G_{1}, \quad$ and $\quad D_{2}=I+\left(\Delta G_{2}\right) G_{2}^{-1}, \quad \Delta G_{2}=\widetilde{G}_{2}-G_{2} ;$ 
then $\widetilde{B}=D_{1} B D_{2}^{-1}$. By Theorem 5.2 , we have

$$
\begin{aligned}
\max _{1 \leq i \leq n} \chi\left(\sigma_{i}, \tilde{\sigma}_{i}\right) & \leq \frac{1}{2} \frac{\left\|D_{1}^{*}-D_{1}^{-1}\right\|_{2}+\left\|D_{2}^{-*}-D_{2}\right\|_{2}}{1-\frac{1}{32}\left\|D_{1}^{*}-D_{1}^{-1}\right\|_{2}\left\|D_{2}^{-*}-D_{2}\right\|_{2}}, \\
\sqrt{\sum_{i=1}^{n}\left[\chi\left(\sigma_{i}, \tilde{\sigma}_{i}\right)\right]^{2}} & \leq \frac{1}{2} \frac{\left\|D_{1}^{*}-D_{1}^{-1}\right\|_{\mathrm{F}}+\left\|D_{2}^{-*}-D_{2}\right\|_{\mathrm{F}}}{1-\frac{1}{32}\left\|D_{1}^{*}-D_{1}^{-1}\right\|_{2}\left\|D_{2}^{-*}-D_{2}\right\|_{2}},
\end{aligned}
$$

as were to be shown.

Theorem 11.4 Let all conditions of Theorem 11.1, except $\delta_{12} \delta_{22}<32$, hold. Then

$$
\begin{aligned}
\max _{1 \leq i \leq n} \varrho_{p}\left(\sigma_{i}, \tilde{\sigma}_{i}\right) & \leq \frac{1}{2^{1+1 / p}}\left(\delta_{12}+\delta_{22}\right), \\
\sqrt{\sum_{i=1}^{n}\left[\varrho_{p}\left(\sigma_{i}, \widetilde{\sigma}_{i}\right)\right]^{2}} & \leq \frac{1}{2^{1+1 / p}}\left(\delta_{1 \mathrm{~F}}+\delta_{2 \mathrm{~F}}\right) .
\end{aligned}
$$

Proof: (by the first half of the proof of Theorem 11.3 and by Theorem 5.3)

\section{Conclusions}

We have developed a relative perturbation theory for eigenvalue and singular value variations under multiplicative perturbations. In the theory, extensions of the celebrated Hoffman-Wielandt theorem and Weyl-Lidskii theorem from the classical perturbation theory are made. For this, we introduced two kinds of relative distances $\varrho_{p}$ and $\chi$. Topologically, our new relative distances are equivalent to the classical measurement for relative accuracy, but the new distances have better mathematical properties. It is proved that $\varrho_{p}$ is indeed a metric on $\mathbf{R}$; while $\chi$ is not. Often it is the case that perturbation bounds using $\chi$ are sharper than bounds using $\varrho_{p}$.

Our unifying treatment in this paper covers almost all previously studied cases and yields sharper bounds than existing ones. Our results are applicable immediately to the computations of sharp error bounds in the Demmel-Kahan QR [8, 1990] algorithm and Fernando-Parlett's implementation of the Rutishauser QD algorithm [13, 1994]. Such applications will be published elsewhere.

\section{Appendix.}

\section{A $\varrho_{p}$ is a Metric on $\mathbf{R}$}

In this appendix, we will prove $(3.20)$

$$
\varrho_{p}(\alpha, \gamma) \leq \varrho_{p}(\alpha, \beta)+\varrho_{p}(\beta, \gamma) \text { for } \alpha, \beta, \gamma \in \mathbf{R} .
$$

As a result, we have 
Theorem A.1 $\varrho_{p}$ is a metric on $\mathbf{R}$.

We strongly conjecture that $\varrho_{p}$ is a metric on $\mathbf{C}$. Unfortunately, we are unable to prove it at this point.

Lemma A.1 The following statements are equivalent:

1. $\varrho_{p}(\alpha, \gamma) \leq \varrho_{p}(\alpha, \beta)+\varrho_{p}(\beta, \gamma)$

2. $\varrho_{p}(\xi \alpha, \xi \gamma) \leq \varrho_{p}(\xi \alpha, \xi \beta)+\varrho_{p}(\xi \beta, \xi \gamma)$ for some $0 \neq \xi \in \mathbf{C}$;

3. $\varrho_{p}(\xi \alpha, \xi \gamma) \leq \varrho_{p}(\xi \alpha, \xi \beta)+\varrho_{p}(\xi \beta, \xi \gamma)$ for all $0 \neq \xi \in \mathbf{C}$.

This lemma follows from Property 3 of Proposition 3.1 .

In what follows, we will be working with real numbers. Since $\varrho_{p}$ is symmetric with respect to its two arguments (Property 2 of Proposition 3.1), we may assume, without loss of any generality, that from now on

$$
\alpha \leq \gamma
$$

There are three possible positions for $\beta$ :

$$
\beta \leq \alpha \text { or } \alpha<\beta \leq \gamma \text { or } \gamma<\beta .
$$

Lemma A.2 (3.20) holds for $\alpha \leq \beta \leq \gamma$, and the equality holds if and only if $\beta=\alpha$ or $\beta=\gamma$.

A proof of this lemma will be given in $\S A .3$. Two different cases shall be considered, in order to confirm $(3.20)$.

1. $\alpha \gamma \geq 0$.

2. $\alpha \gamma<0$.

\section{A.1 The Case $\alpha \gamma \geq 0$.}

Lemma A.2 shows that (3.20) is true if $\alpha \leq \beta \leq \gamma$. If either $\beta<\alpha$ or $\gamma<\beta$, by Property 8 of Proposition 3.1, we have

$$
\varrho_{p}(\alpha, \gamma) \leq \begin{cases}\varrho_{p}(\alpha, \beta) \leq \varrho_{p}(\alpha, \beta)+\varrho_{p}(\beta, \gamma), & \text { if } \gamma \leq \beta \\ \varrho_{p}(\beta, \gamma) \leq \varrho_{p}(\alpha, \beta)+\varrho_{p}(\beta, \gamma), & \text { if } \beta \leq \alpha\end{cases}
$$

\section{A.2 The Case $\alpha \gamma<0$.}

We may assume $\alpha<0$ and $\gamma>0$ (see assumption (A.1)). Consider the three possible positions (A.2) for $\beta$.

1. $\beta \leq \alpha<0$. In this subcase, $1 / \alpha \leq 1 / \beta<0<1 / \gamma$. By Lemma A.2 and Property 4 of Proposition 3.1, we have

$$
\varrho_{p}(\alpha, \gamma)=\varrho_{p}(1 / \alpha, 1 / \gamma) \leq \varrho_{p}(1 / \alpha, 1 / \beta)+\varrho_{p}(1 / \beta, 1 / \gamma)=\varrho_{p}(\alpha, \beta)+\varrho_{p}(\beta, \gamma)
$$

2. $\alpha \leq \beta \leq \gamma$. This subcase has been taken care of by Lemma A.2.

3. $0<\gamma \leq \beta$. In this subcase, $1 / \alpha<0<1 / \beta \leq 1 / \gamma$. The rest is the same as in subcase 1 above. 


\section{A.3 Proof of Lemma A.2}

By Lemma A.1 and by that swapping $\alpha$ and $\gamma$ does not loss any generality, we may even assume, besides (A.1), that

$$
\alpha \leq|\alpha| \leq \gamma
$$

Inequality (3.20) clearly holds if one of $\alpha, \beta, \gamma$ is zero or $\beta=\alpha$ or $\beta=\gamma$ or $\alpha=\gamma$. So from now on, we assume

$$
\alpha<\beta<\gamma, \text { and } \alpha \neq 0, \beta \neq 0, \gamma \neq 0 .
$$

For $1 \leq p<\infty$

$$
\begin{aligned}
\varrho_{p}(\alpha, \gamma)= & \frac{\gamma-\alpha}{\sqrt[p]{\gamma^{p}+|\alpha|^{p}}}=\frac{\gamma-\beta+\beta-\alpha}{\sqrt[p]{\gamma^{p}+|\alpha|^{p}}}=\frac{\gamma-\beta}{\sqrt[p]{\gamma^{p}+|\alpha|^{p}}}+\frac{\beta-\alpha}{\sqrt[p]{\gamma^{p}+|\alpha|^{p}}} \\
= & \frac{\gamma-\beta}{\sqrt[p]{\gamma^{p}+|\beta|^{p}}}+\frac{\beta-\alpha}{\sqrt[p]{|\beta|^{p}+|\alpha|^{p}}} \\
& +(\gamma-\beta)\left(\frac{1}{\sqrt[p]{\gamma^{p}+|\alpha|^{p}}}-\frac{1}{\sqrt[p]{\gamma^{p}+|\beta|^{p}}}\right) \\
& +(\beta-\alpha)\left(\frac{1}{\sqrt[p]{\gamma^{p}+|\alpha|^{p}}}-\frac{1}{\sqrt[p]{|\alpha|^{p}+|\beta|^{p}}}\right) \\
= & \varrho_{p}(\alpha, \beta)+\varrho_{p}(\beta, \gamma) \\
& +\frac{(\gamma-\beta)\left(|\beta|^{p}-|\alpha|^{p}\right)}{\sqrt[p]{\gamma^{p}+|\alpha|^{p}} \sqrt[p]{\gamma^{p}+|\beta|^{p}}} \cdot \frac{\sqrt[p]{\gamma^{p}+|\beta|^{p}}-\sqrt[p]{\gamma^{p}+|\alpha|^{p}}}{|\beta|^{p}-|\alpha|^{p}} \\
& +\frac{(\beta-\alpha)\left(|\beta|^{p}-\gamma^{p}\right)}{\sqrt[p]{\gamma^{p}+|\alpha|^{p}} \sqrt[p]{|\alpha|^{p}+|\beta|^{p}}} \cdot \frac{\sqrt[p]{|\alpha|^{p}+|\beta|^{p}}-\sqrt[p]{\gamma^{p}+|\alpha|^{p}}}{|\beta|^{p}-\gamma^{p}}
\end{aligned}
$$

Now if $\alpha<\beta \leq|\alpha| \leq \gamma$, then $|\beta|^{p}-|\alpha|^{p} \leq 0$ and $|\beta|^{p}-\gamma^{p}<0$, and thus

$$
\begin{aligned}
& \frac{(\gamma-\beta)\left(|\beta|^{p}-|\alpha|^{p}\right)}{\sqrt[p]{\gamma^{p}+|\alpha|^{p}} \sqrt[p]{\gamma^{p}+|\beta|^{p}}} \cdot \frac{\sqrt[p]{\gamma^{p}+|\beta|^{p}}-\sqrt[p]{\gamma^{p}+|\alpha|^{p}}}{|\beta|^{p}-|\alpha|^{p}} \\
& \quad+\frac{(\beta-\alpha)\left(|\beta|^{p}-\gamma^{p}\right)}{\sqrt[p]{\gamma^{p}+|\alpha|^{p}} \sqrt[p]{|\alpha|^{p}+|\beta|^{p}}} \cdot \frac{\sqrt[p]{|\alpha|^{p}+|\beta|^{p}}-\sqrt[p]{\gamma^{p}+|\alpha|^{p}}}{|\beta|^{p}-\gamma^{p}}<0 .
\end{aligned}
$$

Hence $\varrho_{p}(\alpha, \gamma)<\varrho_{p}(\alpha, \beta)+\varrho_{p}(\beta, \gamma)$. Consider now $|\alpha|<\beta<\gamma$. Then

$$
\begin{aligned}
& \frac{(\gamma-\beta)\left(|\beta|^{p}-|\alpha|^{p}\right)}{\sqrt[p]{\gamma^{p}+|\alpha|^{p}} \sqrt[p]{\gamma^{p}+|\beta|^{p}}} \cdot \frac{\sqrt[p]{\gamma^{p}+|\beta|^{p}}-\sqrt[p]{\gamma^{p}+|\alpha|^{p}}}{|\beta|^{p}-|\alpha|^{p}} \\
& \quad+\frac{(\beta-\alpha)\left(|\beta|^{p}-\gamma^{p}\right)}{\sqrt[p]{\gamma^{p}+|\alpha|^{p}} \sqrt[p]{|\alpha|^{p}+|\beta|^{p}}} \cdot \frac{\sqrt[p]{|\alpha|^{p}+|\beta|^{p}}-\sqrt[p]{\gamma^{p}+|\alpha|^{p}}}{|\beta|^{p}-\gamma^{p}} \\
& \leq \frac{(\gamma-\beta)(\beta-|\alpha|)}{\sqrt[p]{\gamma^{p}+|\alpha|^{p}}}\left(\frac{1}{\sqrt[p]{\gamma^{p}+\beta^{p}}} \cdot \frac{\beta^{p}-|\alpha|^{p}}{\beta-|\alpha|} \cdot \frac{\sqrt[p]{\gamma^{p}+\beta^{p}}-\sqrt[p]{\gamma^{p}+|\alpha|^{p}}}{\beta^{p}-|\alpha|^{p}}\right. \\
&\left.\quad-\frac{1}{\sqrt[p]{|\alpha|^{p}+\beta^{p}}} \cdot \frac{\gamma^{p}-\beta^{p}}{\gamma-\beta} \cdot \frac{\sqrt[p]{|\alpha|^{p}+\beta^{p}}-\sqrt[p]{\gamma^{p}+|\alpha|^{p}}}{\beta^{p}-\gamma^{p}}\right)
\end{aligned}
$$

$<0$. 
The last " $<$ " is true because $\sqrt[p]{\gamma^{p}+\beta^{p}}>\sqrt[p]{|\alpha|^{p}+\beta^{p}} \Rightarrow \frac{1}{\sqrt[p]{\gamma^{p}+\beta^{p}}}<\frac{1}{\sqrt[p]{|\alpha|^{p}+\beta^{p}}}$ and

$$
\begin{aligned}
& 0<\frac{\beta^{p}-|\alpha|^{p}}{\beta-|\alpha|} \leq \frac{\gamma^{p}-\beta^{p}}{\gamma-\beta}, \\
& 0<\frac{\sqrt[p]{\gamma^{p}+\beta^{p}}-\sqrt[p]{\gamma^{p}+|\alpha|^{p}}}{\beta^{p}-|\alpha|^{p}} \leq \frac{\sqrt[p]{|\alpha|^{p}+\beta^{p}}-\sqrt[p]{\gamma^{p}+|\alpha|^{p}}}{\beta^{p}-\gamma^{p}} .
\end{aligned}
$$

These inequalities are consequences of Lemma A.3 below since for $1<p<\infty, f(x)=x^{p}$ is convex and $g(x)=\sqrt[p]{x}$ is concave. So we also have $\varrho_{p}(\alpha, \gamma)<\varrho_{p}(\alpha, \beta)+\varrho_{p}(\beta, \gamma)$ for $|\alpha|<\beta<\gamma$. The proof for the case $p<\infty$ is completed.

When $p=\infty$,

$$
\begin{aligned}
\varrho_{\infty}(\alpha, \gamma) & =\frac{\gamma-\alpha}{\gamma}=\frac{\gamma-\beta}{\gamma}+\frac{\beta-\alpha}{\gamma} \\
& =\frac{\gamma-\beta}{\gamma}+\frac{\beta-\alpha}{\max \{|\alpha|,|\beta|\}}+(\beta-\alpha)\left(\frac{1}{\gamma}-\frac{1}{\max \{|\alpha|,|\beta|\}}\right) \\
& <\varrho_{\infty}(\alpha, \beta)+\varrho_{\infty}(\beta, \gamma),
\end{aligned}
$$

as was to be shown.

Lemma A.3 Suppose functions $f(x)$ and $g(x)$ are defined on the interval $[a, b]$, and suppose $f(x)$ is convex and $g(x)$ concave. Let $\alpha \leq \beta \leq \gamma$. Then

$$
\frac{f(\beta)-f(\alpha)}{\beta-\alpha} \leq \frac{f(\gamma)-f(\beta)}{\gamma-\beta} \quad \text { and } \quad \frac{g(\beta)-g(\alpha)}{\beta-\alpha} \geq \frac{g(\gamma)-g(\beta)}{\gamma-\beta}
$$

A proof of this lemma can be found in most Mathematical Analysis books. Intuitively, the two inequalities in Lemma A.3 are well explained by Figure 1.

Acknowledgement: I thank Professor W. Kahan for his consistent encouragement and support, Professor J. Demmel for helpful discussions on open problems in this research area and Professor B. N. Parlett for drawing my attention to Ostrowski theorem. I also thank Drs. Ming Gu, Huan Ren and Yuhua Wu for helping me to prove whether $\varrho_{2}$ is a metric on R. The proof of Lemma A.2 was discovered only after Drs. Ming Gu and Yuhua Wu showing me their proof for $\varrho_{2}$.

\section{References}

[1] J. Barlow and J. Demmel. Computing accurate eigensystems of scaled diagonally dominant matrices. SIAM Journal on Numerical Analysis, 27:762-791, 1990.

[2] F. L. Bauer and C. T. Fike. Norms and exclusion theorems. Numerische Mathematik, 2:137$141,1960$.

[3] R. Bhatia. Perturbation Bounds for Matrix Eigenvalues. Pitman Research Notes in Mathematics. Longmann Scientific \& Technical, Harlow, Essex, 1987. Published in the USA by John Wiley. 

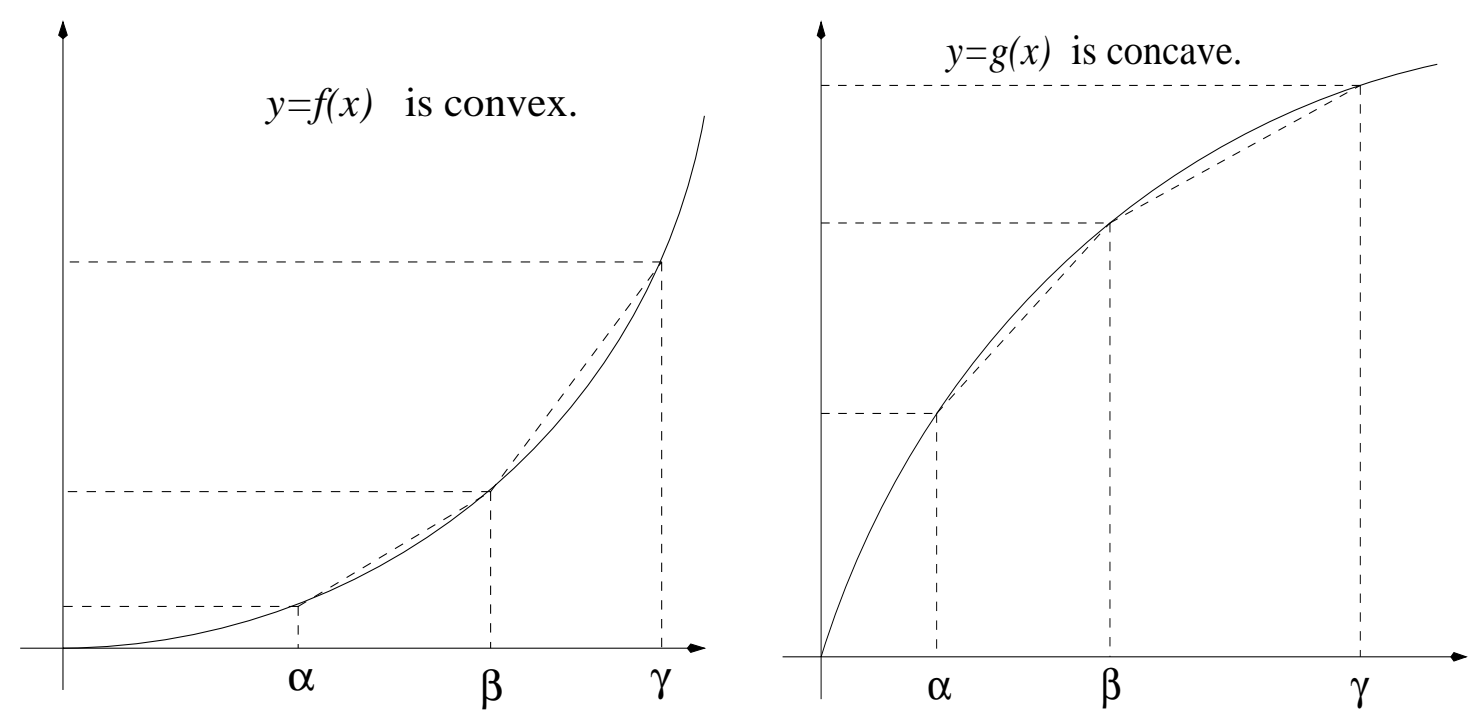

Figure 1: Explaining inequalities for convex and concave functions.

[4] R. Bhatia, C. Davis, and F. Kittaneh. Some inequalities for commutators and an application to spectral variation. Aequationes Mathematicae, 41:70-78, 1991.

[5] G. D. Birkhoff. Tres observaciones sobre el algebra lineal. Universidad Nacional de Tucuman Revista, Serie A, 5:147-151, 1946.

[6] P. Deift, J. Demmel, L.-C. Li, and C. Tomei. The bidiagonal singular value decomposition and Hamiltonian mechanics. SIAM Journal on Numerical Analysis, 28:1463-1516, 1991.

[7] J. Demmel and W. Gragg. On computing accurate singular values and eigenvalues of matrices with acyclic graphs. Linear Algebra and its Application, 185:203-217, 1993.

[8] J. Demmel and W. Kahan. Accurate singular values of bidiagonal matrices. SIAM Journal on Scientific and Statistical Computing, 11:873-912, 1990.

[9] J. Demmel and K. Veselić. Jacobi's method is more accurate than QR. SIAM Journal on Matrix Analysis and Applications, 13(4):1204-1245, 1992.

[10] S. C. Eisenstat and I. C. F. Ipsen. Relative perturbation techniques for singular value problems. Research Report YALEU/DCS/RR-942, Department of Computer Science, Yale University, 1993.

[11] S. C. Eisenstat and I. C. F. Ipsen. Relative perturbation bounds for eigenspaces and singular vector subspaces. In J. G. Lewis, editor, Proceedings of the Fifth SIAM Conference on Applied Linear Algebra, pages 62-66, Philadelphia, 1994. SIAM Publications.

[12] L. Elsner and S. Friedland. Singular values, doubly stochastic matrices, and applications. Linear Algebra and its Applications, 220:161-169, 1995.

[13] K. V. Fernando and B. N. Parlett. Accurate singular values and differential qd algorithms. Numerische Mathematik, 67:191-229, 1994.

[14] G. H. Golub and C. F. Van Loan. Matrix Computations. Johns Hopkins University Press, Baltimore, Maryland, 2nd edition, 1989. 
[15] M. Gu and S. C. Eisenstat. Relative perturbation theory for eigenproblems. Research Report YALEU/DCS/RR-934, Department of Computer Science, Yale University, 1993.

[16] A. J. Hoffman and H. W. Wielandt. The variation of the spectrum of a normal matrix. Duke Mathematical Journal, 20:37-39, 1953.

[17] R. A. Horn and C. R. Johnson. Matrix Analysis. Cambridge University Press, Cambridge, 1985.

[18] W. Kahan. Accurate eigenvalues of a symmetric tridiagonal matrix. Technical Report CS41, Computer Science Department, Stanford University, Stanford, CA, 1966. (revised June 1968).

[19] W. Kahan. Inclusion theorems for clusters of eigenvalues of Hermitian matrices. Technical report, Computer Science Department, University of Toronto, 1967.

[20] W. Kahan. Spectra of nearly Hermitian matrices. Proceedings of the American Mathematical Society, 48:11-17, 1975.

[21] G. Di Lena, R. I. Peluso, and G. Piazza. Results on the relative perturbation of the singular values of a matrix. BIT, 33:647-653, 1993.

[22] R.-C. Li. Bounds on perturbations of generalized singular values and of associated subspaces. SIAM Journal on Matrix Analysis and Applications, 14:195-234, 1993.

[23] R.-C. Li. Norms of certain matrices with applications to variations of the spectra of matrices and matrix pencils. Linear Algebra and its Application, 182:199-234, 1993.

[24] R.-C. Li. Relative perturbation theory: (ii) eigenspace and singular subspace variations. Technical Report UCB//CSD-94-856, Computer Science Division, Department of EECS, University of California at Berkeley, 1994. (revised January 1996).

[25] R.-C. Li. Eigenvalues of symmetrizable matrices. Manuscript, Oak Ridge National Laboratory, 1996.

[26] V. B. Lidskii. The proper values of the sum and product of symmetric matrices. Doklady Akademii Nauk SSSR, 75:769-772, 1950. In Russian. Translation by C. Benster available from the National Translation Center of the Library of Congress.

[27] K. Loewner. Über monotone matrixfunktionen. Math. Z., 38:177-216, 1934.

[28] T.-X. Lu. Perturbation bounds of eigenvalues of symmetrizable matrices. Numerical Mathematics: a Journal of Chinese Universities, 16:177-185, 1994. In Chinese.

[29] R. Mathias. Spectral perturbation bounds for graded positive definite matrices. Manuscript, Department of Mathematics, College of William \& Mary, 1994.

[30] R. Mathias and G. W. Stewart. A block QR algorithm and the singular value decomposition. Linear Algebra and Its Applications, 182:91-100, 1993.

[31] L. Mirsky. Symmetric gauge functions and unitarily invariant norms. Quarterly Journal of Mathematics, 11:50-59, 1960.

[32] A. M. Ostrowski. A quantitative formulation of Sylvester's law of inertia. Proc. National Acad. Sciences (USA), 45:740-744, 1959.

[33] B. N. Parlett. The Symmetric Eigenvalue Problem. Prentice-Hall, Englewood Cliffs, New Jersey, 1980.

[34] I. Slapničar. Accurate Symmetric Eigenreduction By a Jacobi Method. PhD thesis, der Fernuniversität - Gesamthochschule - Hagen, des Facbereichs Mathematik, 1992. 
[35] G. W. Stewart and J.-G. Sun. Matrix Perturbation Theory. Academic Press, Boston, 1990.

[36] J.-G. Sun. The perturbation bounds of generalized eigenvalues of a class of matrix-pairs. Math. Numer. Sinica, 4:23-29, 1982. In Chinese.

[37] J.-G. Sun. Perturbation analysis for the generalized singular value decomposition. SIAM Journal on Numerical Analysis, 20:611-625, 1983.

[38] J.-G. Sun. On the perturbation of the eigenvalues of a normal matrix. Math. Numer. Sinica, 6:334-336, 1984. In Chinese.

[39] C. F. Van Loan. Generalizing the singular value decomposition. SIAM Journal on Numerical Analysis, 13:76-83, 1976.

[40] H. Weyl. Das asymptotische Verteilungsgesetz der Eigenwert linearer partieller Differentialgleichungen (mit einer Anwendung auf der Theorie der Hohlraumstrahlung). Mathematische Annalen, 71:441-479, 1912.

[41] H. Wielandt. An extremum property of sums of eigenvalues. Proceedings of the American Mathematical Society, 6:106-110, 1955.

[42] J. H. Wilkinson. The Algebraic Eigenvalue Problem. Clarendon Press, Oxford, England, 1965.

[43] Z. Zhang. On the perturbation of the eigenvalues of a non-defective matrix. Math. Numer. Sinica, 6:106-108, 1986. In Chinese. 